\title{
Fabrication of TiNi thin film stents
}

Dissertation

zur Erlangung des akademischen Grades

Doktor der Ingenieurwissenschaften (Dr.-Ing.)

der Technischen Fakultät

der Christian-Albrechts-Universität zu Kiel

Lima de Miranda, Rodrigo

Kiel

Juli 2009 
1. Gutachter

2. Gutachter

(ggf. 3. Gutachter)

Datum der mündlichen Prüfung ................................ 
I would like to dedicate this work to my family and especially to my wife 


\section{Contents}

$\begin{array}{lll}1 & \text { Introduction } & 1\end{array}$

2 Fundamentals $\quad 4$

2.1 Martensite phase transformation . . . . . . . . . . . . . . . . . . . . 4

$2.1 .1 \quad$ Definition of martensitic transformation . . . . . . . . . . . . . . 4

2.1 .2 Thermodynamic aspects on martensite transformation . . . . . . 5

2.1.3 Stress induced martensitic transformation and superelasticity . . . 7

2.2 Mechanical properties . . . . . . . . . . . . . . . . . . . 10

2.2 .1 Shape memory effect . . . . . . . . . . . . . . . . . . . . . 10

2.3 TiNi shape memory alloys . . . . . . . . . . . . . . . . . . . . . . 11

2.4 Fabrication of thin films . . . . . . . . . . . . . . . . . . . . . . . . 14

2.4 .1 Magnetron sputtering . . . . . . . . . . . . . . . . . 14

2.4 .2 UV lithography $\ldots \ldots \ldots \ldots \ldots$. . . . . . . . . . . . . . . . 21

2.4 .3 Etching process . . . . . . . . . . . . . . . . . . . . 24

2.5 Production and limitations of standard TiNi stents . . . . . . . . . . 26

2.6 State-of-art on cylindrical deposition/lithography . . . . . . . . . . . . . 30

3 Experimental Techniques 36

3.1 Standard characterization techniques . . . . . . . . . . . . . . . . 36

3.1.1 Scanning Electron Microscope - SEM and Energy Dispersive X-ray Spectroscopy - EDX . . . . . . . . . . . . 36

$3.1 .2 \quad$ Differential Scanning Calorimetry - DSC . . . . . . . . . . . . . . 37

$3.1 .3 \quad$ X-ray Diffraction - XRD . . . . . . . . . . . . . . . . . . . . 38

3.1 .4 Transmission Electron Microscopy - TEM . . . . . . . . . . . . . 40

3.1 .5 Profilometer . . . . . . . . . . . . . . . . . . . . 41

3.1 .6 Tensile test . . . . . . . . . . . . . . . . . . . . 41

3.2 Fabrication techniques . . . . . . . . . . . . . . . . . . . . . . . . . . . . . . . . . . . . . .

3.2.1 Magnetron sputtering. . . . . . . . . . . . . . . . . . . . . . . . . . . . . . . . 42

3.2 .2 UV lithography . . . . . . . . . . . . . . . . . . . . . . . . 42

3.2.3 Rapid Thermal Annealing - RTA . . . . . . . . . . . . . . . . . . 42

4 Results and Discussion $\quad 43$

4.1 Fabrication and characterization of planar thin films . . . . . . . . . . . . 43

$4.1 .1 \quad$ Sputtering device characterization . . . . . . . . . . . . . . . . 43

4.1 .2 Thin film ex situ crystallization and characterization . . . . . . . 48

4.2 Fabrication and characterization of cylindrical thin films . . . . . . . . . 54 
$4.2 .1 \quad$ Sputtering device characterization . . . . . . . . . . . . . . 54

$4.2 .2 \quad$ Cylindrical thin film ex situ crystallization . . . . . . . . . . . . . 63

4.2.3 Mechanical characterization of cylindrical thin films . . . . . . . . 68

4.3 Structuring cylindrical TiNi thin films . . . . . . . . . . . . . . . . . 75

4.3 .1 Cylindrical lithography . . . . . . . . . . . . . . . . . . . . . . . . . . . . . . . . . . . . . .

4.3 .2 Wet etching . . . . . . . . . . . . . . . . . . 85

4.3.3 Method used to fabricate fine structured TiNi "thick" films by magnetron sputtering . . . . . . . . . . . . . . . . . . . 98

$\begin{array}{lll}5 & \text { Summary } & 102\end{array}$

$\begin{array}{lll}6 & \text { Acknowledgments } & 105\end{array}$

$\begin{array}{lll}7 & \text { Own publications and patent applications } & 107\end{array}$

\begin{tabular}{ll}
\hline Bibliography & 117
\end{tabular} 



\section{Chapter 1}

\section{Introduction}

The term stent came from a dentist, called Dr. T.C. Stent. He developed in the late 1800's a dental device to support and assist formation of teeth impression. This term is used today for devices used to scaffold or brace the inside of tubular passages or lumens, e.g. esophagus, biliary duct, and most importantly, a series of blood vessels, e.g. coronary, carotid, iliac, aorta, femoral arteries, etc. A stent in a cardiovascular system is most often used as a complement to balloon angioplasty. Balloon angioplasty is a procedure where a balloon is placed in the diseased vessel and expanded in order to re-open a clogged lumen. This disease is called stenosis. Stents are also used to support grafts in the aneurysms treatment. An aneurysm is caused by the weakening of an arterial wall, which presents a risk of rupture. In this case, surgery repairs are difficult. With a endovascular approach (non-surgically) a graft is placed in the health part of the artery through the aneurysm. Preventing the aneurysm from growth and rupture. There are two kind of stents: balloons expandable stents, which have the ability to undergo plastic deformation, maintaining the desired size after deployment and self-expanding stents which should have sufficient elasticity to be compressed for delivery and then expanding in the target area. An ideal stent, as described for numerous groups [1, 2, 3] should have: low profile in order to be easily crimped into catheter; sufficient radial hoop force and negligible recoil to overcome the forces imposed by the atherosclerotic arterial wall; adequate radiopacity to assist the stent placement; thromboresistivity, the 
material must be blood compatible; drug delivery capacity and fatigue resistance.

A. Oelander, in 1932, has discovered superelasticity (or pseudoelasticity) on Au-Cd alloy, as described by Otzuka and Wayman [4]. Greninger and Mooradian observed the formation and disappearance of a martensitic phase by decreasing and increasing the temperature of a Cu-Zn alloy. In the early 1960s, Buehler and his co-workers at the U.S. Naval Ordinance Laboratory discovered the shape memory effect in TiNi. Since then TiNi superelasticity has been used in medical application. TiNi material combines the ideal stent cited properties, which turns it to the material of choice for many vascular applications.

Conventionally - starting from a bulk material - fabricated stents show a relatively large and homogeneous wall thickness exceeding $80 \mu \mathrm{m}$. The fabrication of smaller and more flexible stents with optimized properties, e.g. prevention of restenosis, reduction of the device profile allowing the access to small location, increase the durability, increase the radiopacity, is a challenging and desired task. Deposited TiNi thin films are well known for their comparable mechanical properties to TiNi bulk material [5] and to be less thrombogenic compared to conventionally TiNi bulk material due to their extremely pure composition [6].

The success of stents and other medical implants, which conventionally consist of thin walled, laser structured tubes based on superelastic TiNi on the one hand, and the limitations regarding the miniaturization of these medical devices on the other hand, has motivated the application of thin film technology as an attractive alternative approach for some applications, e.g. devices for small vessels.

In accordance with recent trends and meeting the demand for semi-finished products based on thin film technology, this thesis describes a fabrication routine for thin walled, structured cylindrical TiNi thin films in three steps, as well as the characterization of structured cylindrical TiNi thin films. Magnetron sputtering was used to deposit cylindrical superelastic TiNi films [7, 8]. The cylindrical deposition presents many challenges, e.g. film growth on non-planar surfaces, film composition, thickness distribution and mechanical properties. All these issues are addressed in the course of this disser- 
tation. A cylindrical lithography device based on the synchronization of a planar mask with the rotation of the cylindrical substrate were developed and characterized. This device allows the patterning of complex geometries on the surface of a cylindrical sample and was successfully employed to pattern cylindrical TiNi thin film. The last fabrication process is wet etching. The influence of the etchant movement as well as its composition were investigated and optimized in order to reduce the influence of the wet etching isotropic behavior on the feature size resolution.

Mechanical properties of cylindrical TiNi films were investigated by tensile testing and a self developed hoop force test. Cylindrical TiNi films showed a superelastic strain up to $6.5 \%$ in tensile testing experiments. The design limitation due to wet etching isotropic behavior motivated the development of an alternative approach to fabricate "thick" films with a very small mesh. This method is a promising substitute for the conventional one, once the aspect ratio of this method is comparable to the 10:1 ratio achieved by laser cut technology [9]. 


\section{Chapter 2}

\section{Fundamentals}

\subsection{Martensite phase transformation}

\subsubsection{Definition of martensitic transformation}

Martensitic transformation is the term given, according to the German metallurgist Adolf Martens (1850-1914), to a displacive and diffusionless first order transformation in solids, where atoms cooperate between itself, often due to shear stress, in order to form a new crystallographic structure as shown in figure 2.1. At first, Martens observed this tranformation in different steels. Since then, martensitic transformation has been found in many materials, i.e. shape memory alloys. The high temperature phase is called the parent phase and the low martensite. The parent and martensite phase are separated by a plane called habit. The transformation exhibits hysteresis and generally is adiabatic and athermal.

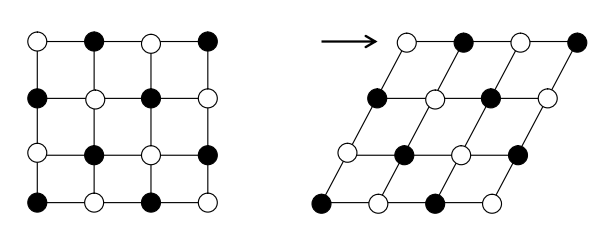

Figure 2.1: Schematic diagram of martensite transformation

The phenomenological martensite transformation (MT) theory proposed by 
Wechsler et al. [10] and by Bowles et al. [11, 12] consider the invariant plane strain (IPS) as a superposition of the following components: a lattice invariant shear (LIS), a lattice strain (Bain strain) rotation and a dilatation. The martensitic transformation is a first order transformation, consequently it proceeds by nucleation and growth. Since martensitic transformation is associated with a shape change, a large strain arises around the martensite when it is formed in the parent phase. The stress reduction is important for nucleation and growth processes. Two ways are possible to reduce the latice stress: introduction of twins or slip, as shown in figure 2.2. This twins and slip are called lattice invariant shears, since neither process change the structure of the martensite. The formation of twins is directly related to shape memory effect (SME), superelasticity and stress induced martensite transformation.

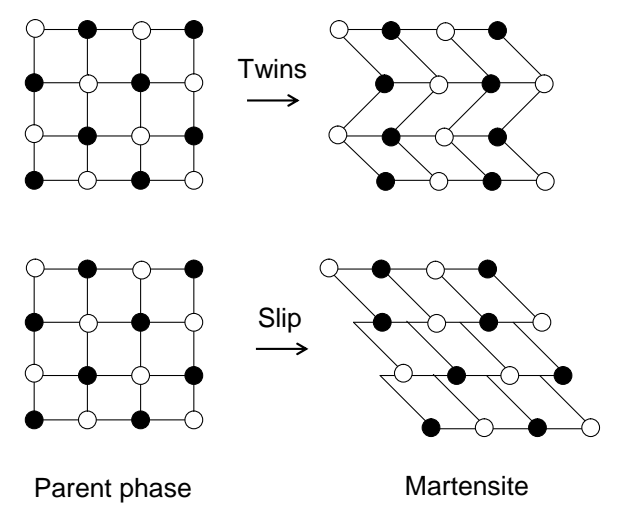

Figure 2.2: Schematic diagram of martensite adjustment: twins and slip

\subsubsection{Thermodynamic aspects on martensite transformation}

The driving force (Df) for the spontaneous martensite transformation, as shown in figure 2.3, is the difference in the chemical free energy between parent and martensite phases. The transformation did not occur at the equilibrium temperature $T_{o}$. The transformation requires an excess of free energy to overcome strain and interface energy due to nucleation of the new phase. Thus, if the free energy is greater than the required driving force, the transformation will then start. 


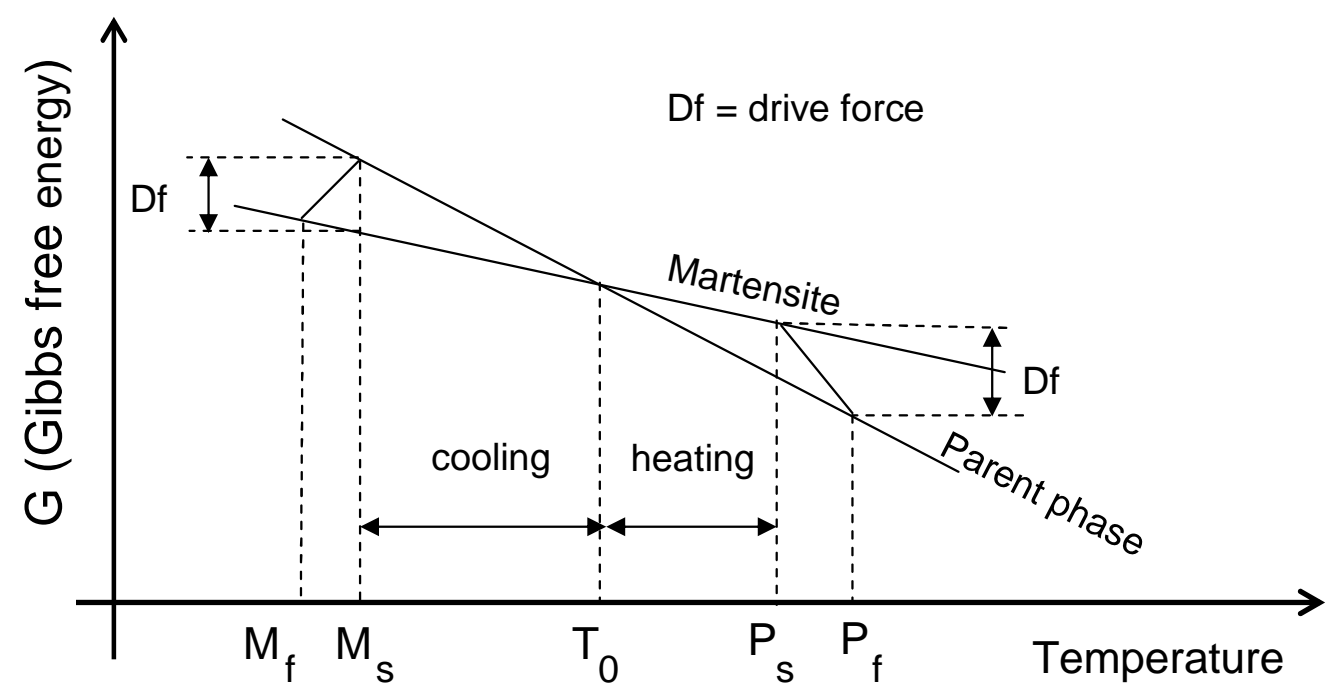

Figure 2.3: Representation of Gibbs free energy curves for parent and martensitic phases and their relations to the martensite transformation temperatures.

If the specimen is not cooled to $M_{s}$ (martensite start) below $T_{o}$, the nucleation of a martensite will not start. A driving force is also necessary for the reverse transformation: the specimen must be overheated to $P_{s}$ (parent phase start) above $T_{o}$ in order to reverse the transformation returning to the parent phase. The temperature range between $M_{s}$ and $M_{f}$ (martensite finish) and $P_{s}$ and $P_{f}$ (parent phase finish) is related to the thermoelasticity theory originally described by Kurdjumov and Khandros [13]. The temperature differences between the martensite nucleation and the parent phase transformation represent the formation of hysteresis. Variants of martensite hysteresis loop according to Paskal and Monasevich [14] are shown in figure 2.4.

The martensitic hysteresis loop under thermoelastic transformation is dominated by elastic and dissipative energy. The thermoelastic hysteresis loop is formed when the dissipative energy tends to zero and the elastic strain energy modifies the equilibrium temperature between the martensite and the parent phase. Figure 2.4 (a) shows the martensite hysteresis loop where only dissipative energy contributes to the transformation. The hysteresis width is characterized by the amount of the dissipative energy 


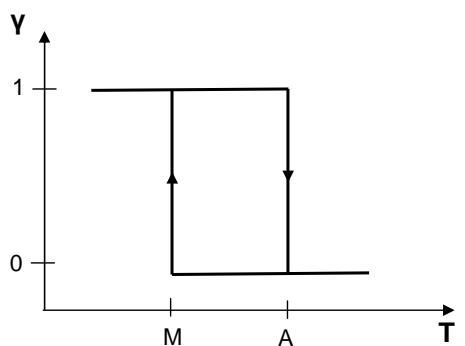

(a)

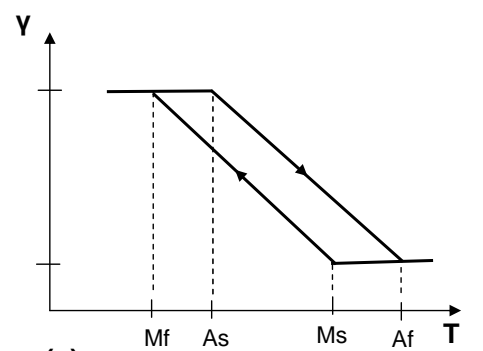

(c)

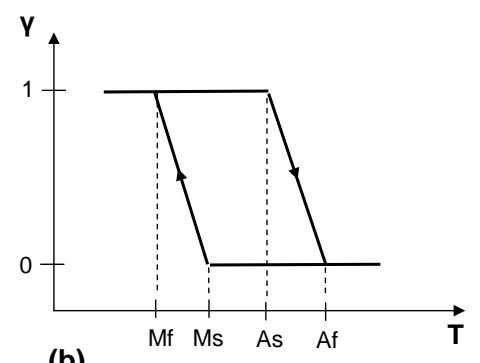

(b)

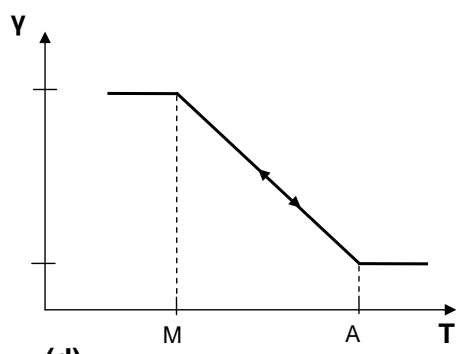

(d)

Figure 2.4: Variants of martensite hysteresis loop after Paskal and Monasevich [14]: (a) only dissipative energy (b) elastic and dissipative energy contributions (dissipative > elastic) (c) elastic and dissipative energy contributions (dissipative < elastic) (d) only elastic energy contributions.

contribution during the transformation, as shown in figure 2.4 (b) and 2.4 (c). Figure 2.4 (d) shows a complete thermoelastic transformation path, where variation on the temperature produces a volume increase or decrease of the martensite.

\subsubsection{Stress induced martensitic transformation and superelasticity}

As mentioned in section 2.1.2 martensitic transformation occurs by cooling below $M_{s}$ temperature, but it can also be induced above $M_{s}$ by an applied stress. Usually, to achieve the so called stress induced martensite transformation (SIM), the shape memory alloy has to be in a stable parent phase. At a critical stress, the martensite is more stable than the parent phase, thus the structure reverts to martensite and a plateau stress is obtained. The stress will shift the transformation temperature and the variant formation 
will have the same orientation as the applied stress. It has been found [15] that above $M_{s}$ the stress required to induce the martensite transformation increases linearly with the temperature.

This linearity can be described by the Clausius-Clapeyron equation [16].

$$
\frac{d \sigma}{d T_{M s}}=\frac{\rho \cdot \Delta H_{p \rightarrow m}}{\epsilon_{p \rightarrow m} \cdot T_{M s}}
$$

where $\sigma$ is the applied uniaxial stress, $\Delta H_{p \rightarrow m}$ is the mass entalphy of the transformation per unit of volume, $\epsilon_{p \rightarrow m}$ is the transformation strain in the direction of the applied stress and $T_{M s}$ is the martensite starting temperature. The strain will be induced in direction of the applied stress.

If the temperature is above $P_{f}$ and the stress decreases, the martensite shrinks and the initial shape is recovered, this phenomenon is called superelasticity.

In general, pseudoelasticity refers to the non-linear unloading characteristics that are observed, whereas superelasticity refers to materials which exhibit a stress plateau and an inflection point upon unloading. An example of a superelastic stress-strain curve is shown in figure 2.5 .

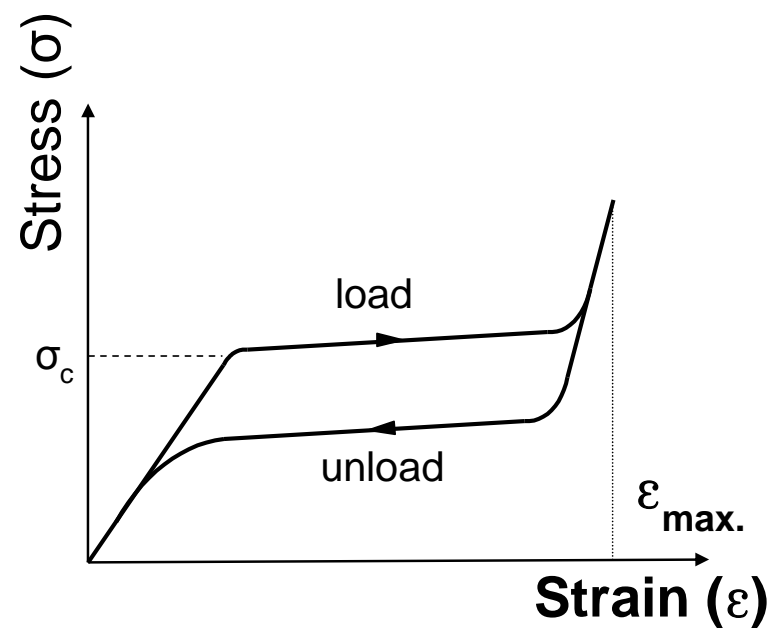

Figure 2.5: Superelastic effect observed in shape memory alloys. $\sigma_{c}$ is the critical stress necessary to start martensite transformation. 
This curve shows the loading and unloading stresses as well as the critical stress $\sigma_{c}$ and the maximum strain $\epsilon_{\max }$. The martensite transformation starts when the increasing load achieves the critical stress $\sigma_{c}$. After the transformation is complete the martensite can still be elastically deformed. If the applied stress is released before the martensite is plastically deformed, the deformation will be totally reversed.

Superelasticity usually occurs within a very small temperature window between $P_{f}$ and $T, T$ being the highest temperature that is possible to form martensite, as shown in a stress-temperature diagram, figure 2.6. This temperature window is between 50-100 $\mathrm{K}$ wide [17]. Above $T$, the critical stress for inducing martensite is too high to move dislocations and, thus, no more stress induced martensite can be formed. The reason is that martensite becomes stable at temperatures above $T$.

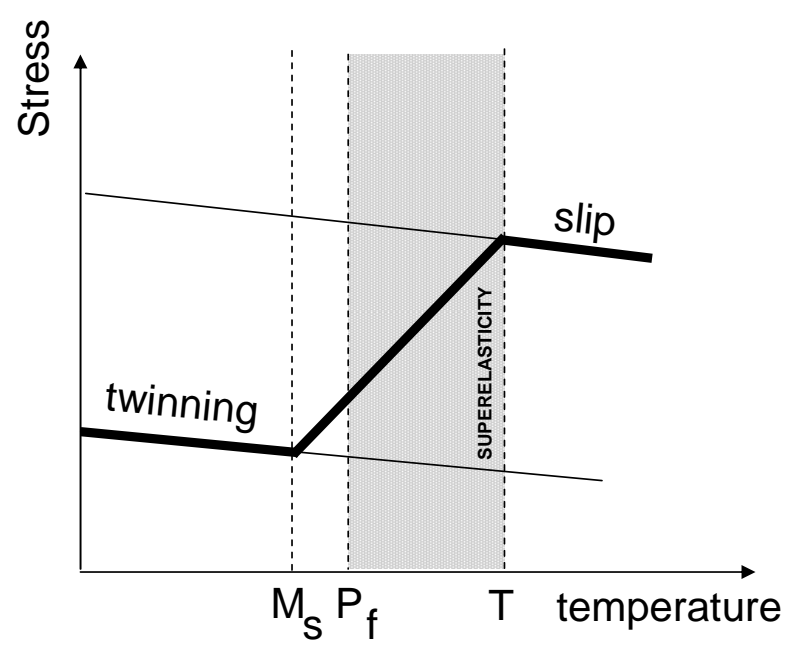

Figure 2.6: Stress-temperature diagram [15], where $M_{s}$ is the martensite start, $P_{f}$ parent phase finish and E highest temperature that is possible to form martensite.

Martensite is most stable and is formed easily below $P_{f}$. This is the region where the thermal shape memory effect is observed. Between $P_{f}$ and $E$ is where the superelastic effect occurs. Above the critical stress required to induce martensite, no further transformations occur. When a shape memory alloy is heated above its transformation temperature it becomes austenitic. If a stress is then applied to the alloy in this 
state, large deformation strains can be obtained and stress induced martensite is formed. Upon removal of the stress, the martensite reverts to its parent phase. The deformation characteristics of the non-linear stress induced martensite transformation show that if $\mathrm{T}>E$, then austenite is deformed plastically in slip. If $\mathrm{T}<M_{s}$, deformation is due to the motion of martensitic twin boundaries. For both cases, low recoverable strains are obtained when unloaded.

\subsection{Mechanical properties}

\subsubsection{Shape memory effect}

A typical solid material presents two kinds of deformation: elastic and plastic. Elastic deformation is a change in shape of a specimen that is reversible after the applied force is removed. This case of deformation involves stretching of the bonds. Figure 2.7 (1) shows the elastic region of a material upon applied stress.

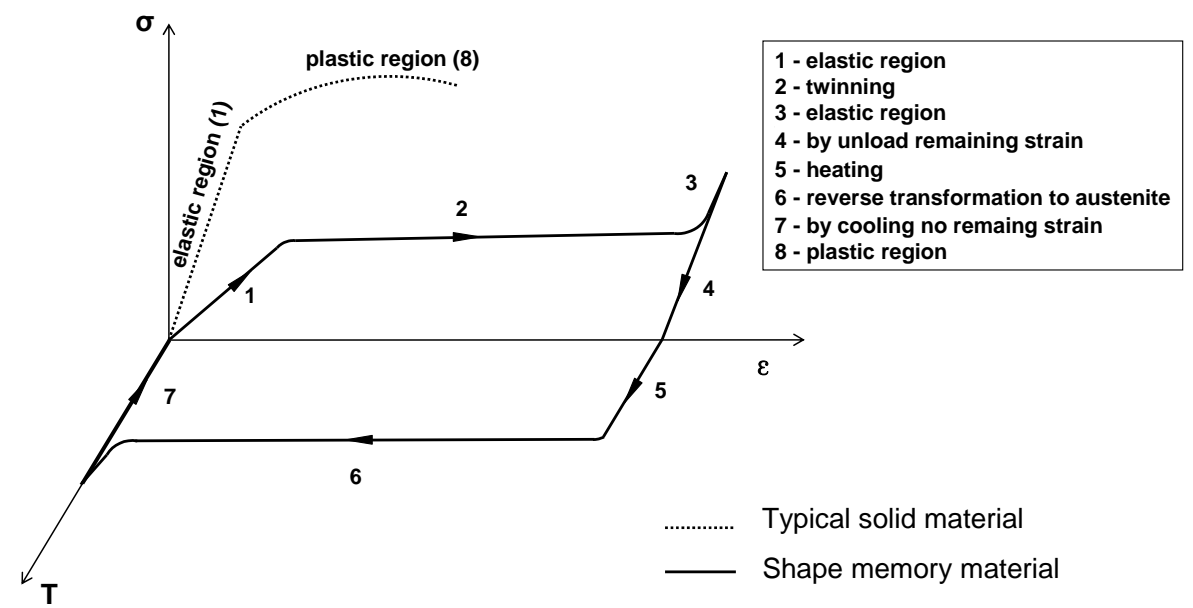

Figure 2.7: Schematic of classic solid material stress strain curve and shape memory effect curve as function of stress, strain and temperature.

In the case of plastic deformation $2.7(8))$ the specimen is deformed permanently and it is unable to return to its initial shape. The Shape Memory Effect (SME) is a phenomenon where the specimen is deformed below $M_{f}$ or at temperatures between 
$M_{f}$ and $P_{s}$ by an applied stress (tension, compression or bending) and returns to its original shape upon heating to a temperature above $P_{f}$ as shown figure 2.7. Figure 2.8 illustrates the crystallographic arrangement during martensitic transformation.

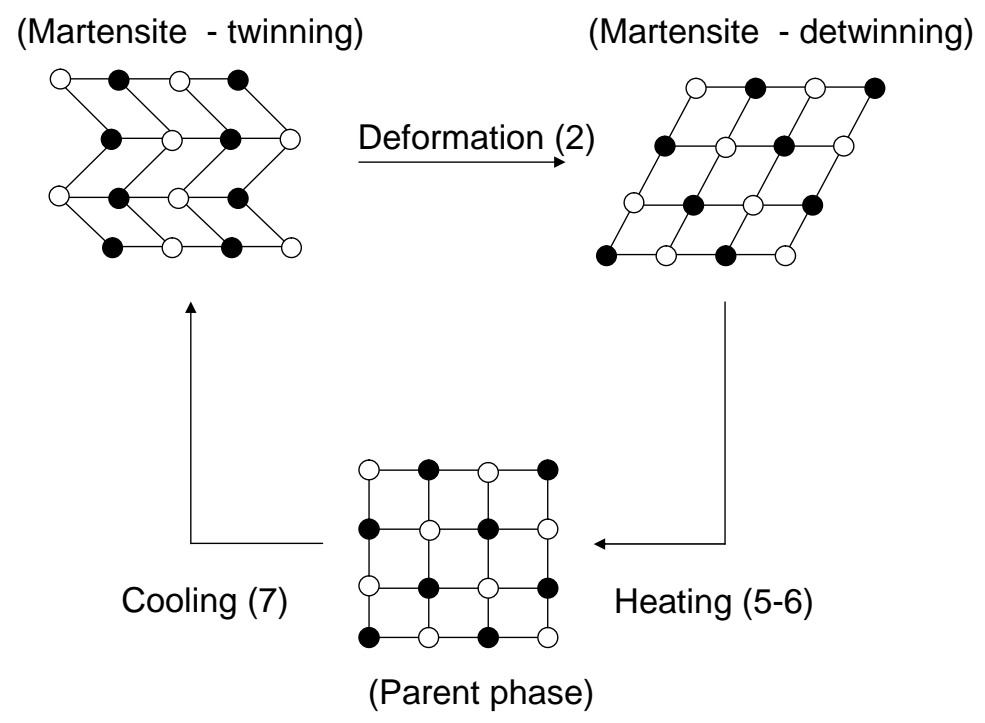

Figure 2.8: Crystallographic arrangement during martensitic transformation.

Figure 2.7 shows a SME stress-strain curve as a function of temperature. At first the specimen shows an elastic deformation, figure 2.7 (1), until the critical stress is achieved. The martensite transformation starts through the twinning, figure 2.7 (2). After the seemingly complete plastic martensitic deformation, figure 2.7 (4), the specimen is heated and the reverse transformation occurs, figure 2.7 (5-7), and the sample completely recovers its initial shape.

\subsection{TiNi shape memory alloys}

NiTi shape memory effect was first observed in 1963 by Buehler et al. [18], who worked at the Naval Ordinance Laboratory. As such NiTi is also known as NiTinol. NiTi exhibits many properties common to other shape memory alloys, for example: the shape memory effect, superelasticity and the two-way effect, but it also demonstrates unique 
characteristics: a low elastic anisotropy, the elastic constant decreases proportionately to the increase in temperature, amorphisation during sputtering, high ductility, corrosion resistance and biocompatibility [19]. The NiTi properties are dependent mainly on its stoichiometry and thermohistory.

There are two possible transformation paths in NiTi shape memory alloys: from B2 cubic parent phase, called austenite, directly to monoclinic B19' when NiTi is fully annealed and from the B2 parent to the R-phase and then to the B19' phase when the specimen was thermo-mechanically treated. The Austenitic or B2 phase is the high temperature phase in NiTi shape memory alloy and has a cubic centered structure and space group $\operatorname{P} m \overline{3} m$ number 221, as shown in figure 2.9 (a).

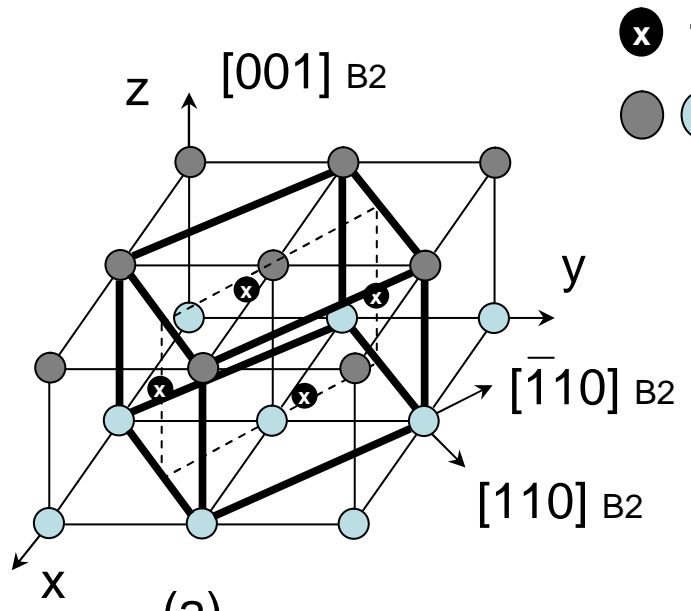

(a)

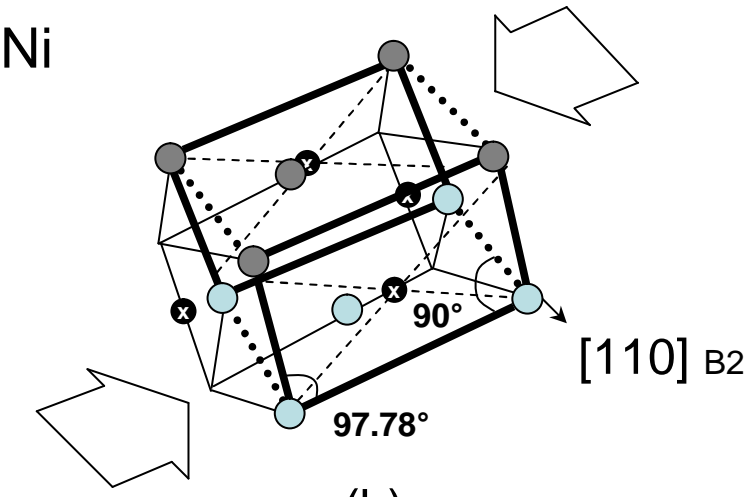

(b)

Figure 2.9: (a) NiTi austenite unit cell structure $(a=b=c=0.3015 \mathrm{~nm})$ [20], (b) NiTi B19' monoclinic unit cell structure, $a=0.2898, b=0.4108$, $c=0.4646 \mathrm{~nm}$ and $\beta=97.78^{\circ}$ according to Ohba [19]

The B19' martensite has a monoclinic structure and it belongs to the space group $P 2_{1} / m$ space group number 11 . Lattice parameters for $T i_{49.2} N i_{50.8}$ atomic percent : a $=$ $0.2898, \mathrm{~b}=0.4108, \mathrm{c}=0.4646 \mathrm{~nm}$ and $\beta=97.78$ and they are composition dependent. Figure 2.9 (b) shows the NiTi B19' martensite structure according to Ohba [19]. The R-phase has a rhomboedric crystal structure and it belongs to the space group P3. The name R-phase was given to that phase because it is formed from a rohmboedric 
distortion of the parent lattice.

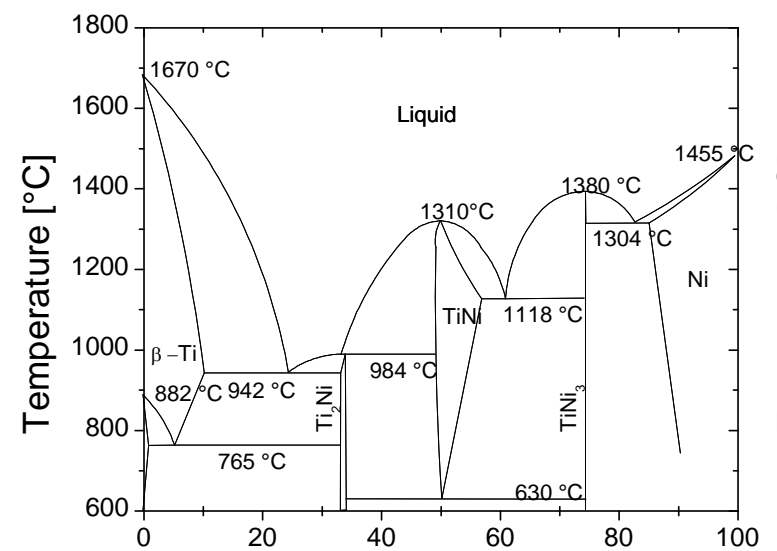

(a)

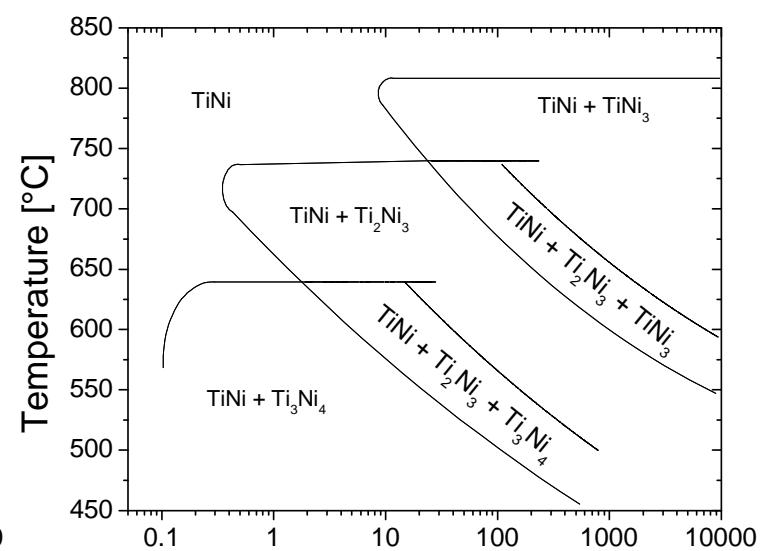

(b)
Annealing time $[\mathrm{Hr}]$

Figure 2.10: (a) TiNi phase diagram [21], (b) TTT diagram describing aging behavior for $\mathrm{Ti}_{52} \mathrm{Ni} i_{48}$ after Nishida et al [22].

The phase diagram is an important tool to understand the shape memory property. TiNi phase diagram (figure 2.10 (a)) shows that the Ni and Ti solubility in the NiTi phase is very narrow, causing high property dependency of the stoichiometry. By increasing the concentration of $\mathrm{Ti}$ the precipitation $T i_{2} N i$ will be formed. By increasing the concentration of $\mathrm{Ni}$ in 0.5 atomic percentage, the precipitation $\mathrm{TiNi} i_{3}$ will be formed. Nishida et al [22] found that the phases $T i_{3} N i_{4}, T i_{2} N i_{3}$ and $T i N i_{3}$, appear depending upon aging temperature and time, as summarized by time-temperature-transformation (TTT) in figure 2.10 (b). The formation of such precipitates helps to adjust the transformation temperatures of the martensite transformation [19].

Several factors influence the martensitic transformation temperature. Figure 2.11 shows how the Ni content influences the martensite start temperature [23]. The transformation temperature is not only influenced by the chemical composition, but also by metallurgical factors as impurities (oxygen and nitrogen) and the grain size. These effects are much weaker than that of chemical composition. Table 2.1 shows the NiTi mechanical and thermal properties. 


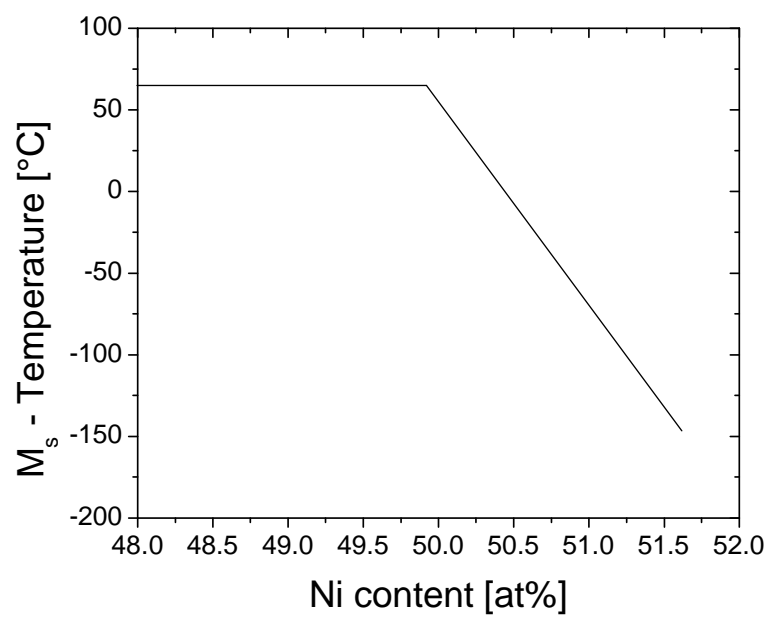

Figure 2.11: The predicted martensite transformation start $\left(M_{s}\right)$ versus Ni content [23].

\begin{tabular}{ccc} 
& Martensite & Austenite \\
\hline Thermal conductivity & $0.086 \mathrm{~W} / \mathrm{cm} \cdot{ }^{\circ} \mathrm{C}$ & $0.18 \mathrm{~W} / \mathrm{cm} \cdot{ }^{\circ} \mathrm{C}$ \\
\hline Thermal expansion & $6.6 \times 10^{-6} /{ }^{\circ} \mathrm{C}$ & $11 \times 10^{-6} /{ }^{\circ} \mathrm{C}$ \\
\hline Young's Modulus & 28 to $41 \mathrm{GPa}$ & $83 \mathrm{GPa}$ \\
\hline
\end{tabular}

Table 2.1: NiTi mechanical and thermical properties [24].

\subsection{Fabrication of thin films}

In this chapter the fundamentals of fabrication and structuring of thin films by means of magnetron sputtering technology, UV lithography and wet etching will be described.

\subsubsection{Magnetron sputtering}

Sputtering is the name given to the result of momentum transfer between gas ions and a matrix atom (target). A plasma is initiated by applying a large voltage between two electrodes placed in a low-pressure gas. Once a plasma is formed, ions in the plasma are accelerated toward the negatively charged cathode (target). When the ions strike the surface of the cathode, they set the cathode material free, as well as realease secondary electrons, which are accelerated away from the cathode. They may collide with neutral 
gas species while crossing from a cathode to anode, ionizing further the gas. Figure 2.12 shows a simple model of some processes that may occur when an ion strikes a cathode surface developed by Wehner and Anderson [25], where chemical effects were ignored and the atoms were treated as spheres.

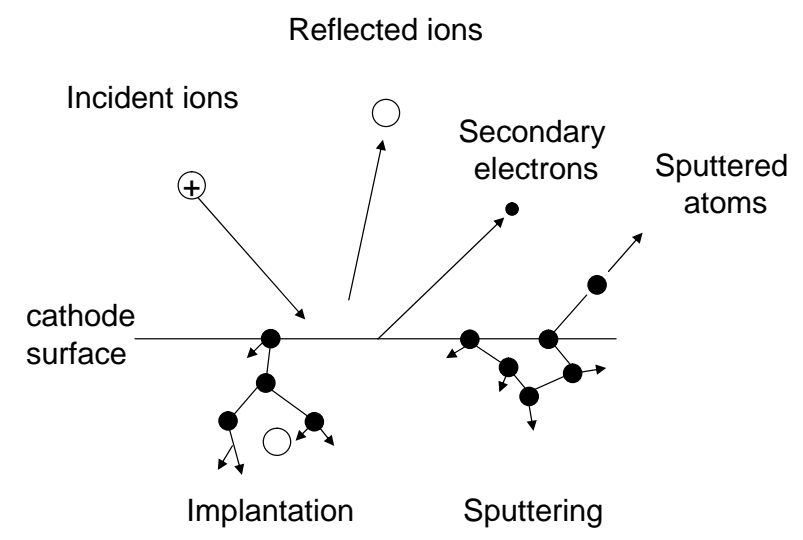

Figure 2.12: Simple model of some processes that may occur when an ion strikes a cathode surface developed by Wehner and Anderson [25].

The sputtering technique is usually divided into four categories: $\mathrm{DC}, \mathrm{RF}$, magnetron and reactive. In the scope of this dissertation magnetron sputtering, DC and RF were used. DC-sputtering is the oldest and simplest sputtering technique and it is limited to electrically conducting materials. In a DC-sputtering a constant potential difference between anode and cathode is applied. The potential difference applied between cathode and anode drops completely in the first milimeters in front of the cathode. This cathode voltage drop sustains the plasma by accelerating secondary electrons emitted for the cathode. Thus, to sustain a DC-discharge, the electrodes have to be conducting. If one or both are non-conductive, e.g deposition of dielectric films, the electrodes will be charged up due to the accumulation of positive or negative charges and the discharge will be extinguish. To over come this problem an alternative volage (RF-sputtering) can be applied between cathode and anode, and the charge accumulated during half cycle will be partially neutralized by the opposite charge accumulated during the next half cycle. In addition to this two glow discharge techniques a magnetic field can also be applied. The most well known type of sputtering characterized by magnetron discharge 
is called magnetron sputtering and can be divided in cylindrical, circular and planar [26]. In planar magnetron sputtering an axisymetric magnetic field is applied with a permanent magnet behind the cathode in such a way that the magnetic field lines start and return at the magnet, as shown in figure 2.13. Thus, a 'magnetic ring' is formed at the cathode surface and will be responsible to 'trap' the electrons that are accelerated away from the cathode. These electrons will move in helices around the magnetic field lines and they will travel a much longer path-length in the plasma than in conventional glow discharges, increasing the ionization collisions and consequently the ion fluxes.

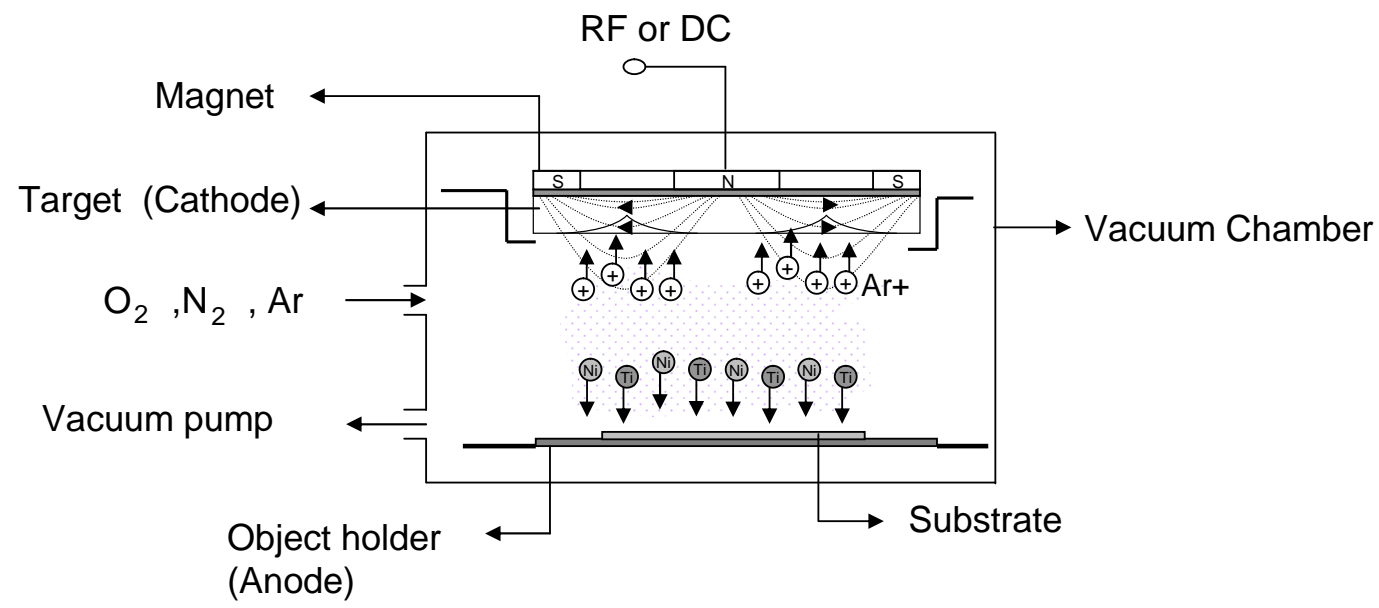

Figure 2.13: Schematic of a magnetron sputtering device.

The sputter deposition rate depends on the ion flux to the target, as well as the transport of the sputtered material across the plasma to the substrate. The ion flux $\left(J_{i o n}\right)$ in a DC plasma can be approximated by the Langmuir-Child relationship:

$$
J_{i o n} \propto \sqrt{\frac{1}{m_{\text {ion }}}} \frac{V^{3 / 2}}{d^{2}}
$$

where $V$ is the voltage difference from the cathode to the elctrode, $d$ is the dark space thickness, which corresponds to the region near to the cathode with strong electric field, and $m_{i o n}$ is the ion mass.

The sputter yields is the number of sputtered atoms divided by the number of incident particles and corresponds to the sputtering efficiency. It depends on the ion mass, 
the ion energy, the target mass and the target crystallinity. The angular dependence of the sputter yields is a strong function of the target material and the incident ion energy. Generally, materials which have high sputter yields have little angular dependency and materials with low sputter yields have high angular dependency [27].

In order to obtain superelastic NiTi films, it is most important to control the composition of the sputtered layer. The sputtering of alloyed NiTi targets reveals a characteristic loss of titanium, as the sputtering yield of $\mathrm{Ni}$ is higher than for $\mathrm{Ti}$. It has been shown, that the composition of the sputtered flux may vary with the polar angle of ejection because of the different angular distribution of the sputtered components [28, 29, 30, 31].

Uniform deposition is important and required in many fields, e.g. microelectronics, optical industry, etc. The film thickness distribution in magnetron sputtering, as described by Soloviev et al. [32, is determined by the equation:

$$
T=\int_{0}^{2 \pi} \int_{0}^{R} \Psi(R) \cos ^{n}(\Theta) R / c^{2} d R d \varphi
$$

where $c^{2}=a^{2}+b^{2}, b^{2}=R^{2}+R^{2}-2 \operatorname{Rr} \cos \varphi$. The geometries are shown in figure 2.14 . The expression $\cos ^{n}(\Theta)$ corresponds to the spatial distribuition of the sputtered atoms flow and the cathode erosion ration is described by the function $\Psi(R)$.

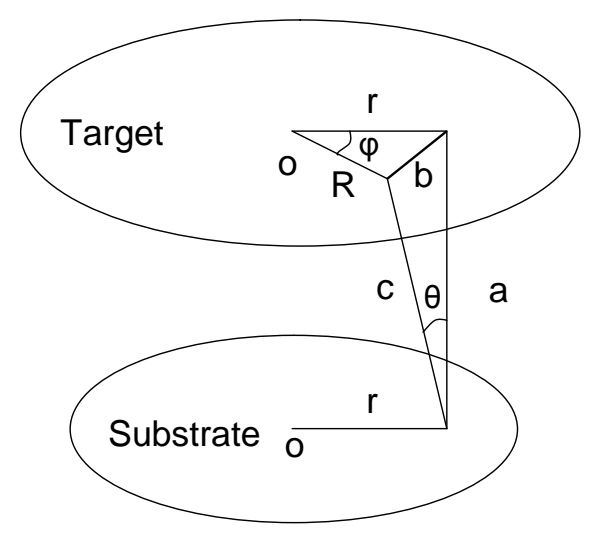

Figure 2.14: Target-substrate geometrical parameters [32].

Sputtered NiTi thin films are commonly deposited in an amorphous state and require a high-temperature annealing step to crystallize them. Their crystallization process is 
driven by the kinetic interplay of both nucleation and growth, and their resulting microstructure dictates the martensitic transformation behavior and actuation properties, as described in the previous section.

Generally, deposition is considered as six sequential steps: (1) adsorption is the approaching of a molecule on the surface, (2) surface diffusion is one of the most important determinatns of the film structure because it allows the adsorbing species to find each other, (3) reaction of the adsorbed species with each other and with the surface to form the bonds of the film, (4) nucleation is the start of the aggregation of the film material and it is strongly influenced by the substrate surface energy. Thus, the film growth can be divided in three modes: Frank-Van der Merwe (layer), VolmerWeber (island) and Stranski-Krastanov. (5) as the film grows, it develops a structure or morphology. Thornton [33] introduced a structure zone model (SZM), which describes the microstructure of magnetron sputtering films based on the relation between substrate temperature and the melting point of the sputtered material $\left(T_{s} / T_{m}\right)$ and also the variation of the inert sputtering gas pressure. Figure 2.15 shows a simplified schematic representation of structure zone model by Thornton.

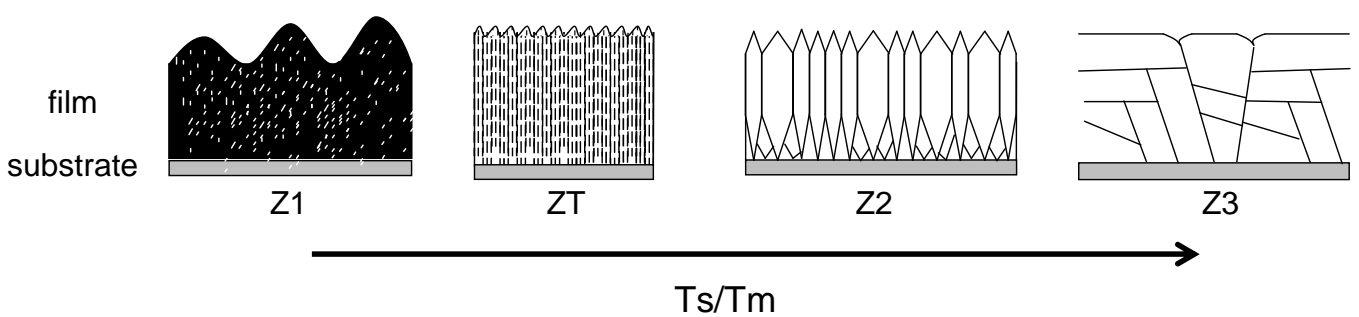

Figure 2.15: Schematic representation of structure zone model by Thornton [33].

The structure zones are directly connected with the mechanical properties of deposited films. Zone 1 , where $T_{s} / T_{m}$ is $<0.1$ at $0.15 \mathrm{~Pa}$ to $<0.5$ at $4 \mathrm{~Pa}$, is a densely packed zone and the deposited films show high hardness and little lateral strength. Zone T $(\mathrm{Ts} / \mathrm{Tm}=0.1-0.4$ at $0.15 \mathrm{~Pa}$ or $0.4-0.5$ at $4 \mathrm{~Pa})$ is the transition zone and is characterized by high dislocation density and the deposited films showing high hardness and strength 
but no ductility. Furthermore, Zone 2, where $T_{s} / T_{m}$ is $0.4-0.7$, is formed by columnar grains and dense grain boundaries and the films are hard with low ductility. Finally, zone 3 is formed by increasing the relation $T_{s} / T_{m}$ to 0.6 to 1 . The zone is formed by large equiaxed grains and a bright surface. The films also show low dislocation density and soft recrystallized grains [33, 34].

The film morphology and consequently their mechanical properties can be influenced by the deposition angle, as discussed by Thornton [35]. Figure 2.16 shows the influence of the incident angle on the film morphology. The film tends to grow forming islands. When $\theta$ is zero the gaps between the insland are easily filled up with further deposition. When the $\theta$ is higher than zero, the islands will act as obstacles, causing then the shadowing effect, forming a void columnar microstrucutre.

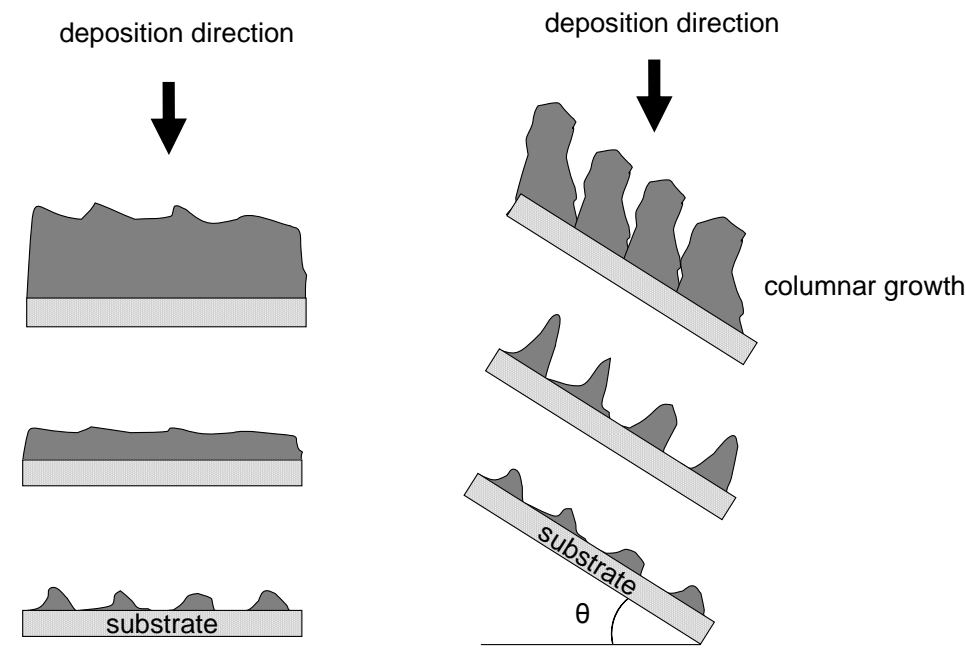

Figure 2.16: A schematic of thin film growth. Shadowing effect due to deposition angle adapted from Thornton [35].

Finally, (6) diffusion interactions occur within the bulk of the film and the substrate.

Residual stresses in a deposited thin film-substrate system is defined as a sum of thermal stress, intrinsic stress and external stress and it is a potential source of problems in many applications. The residual stresses can influence the film characteristics, e.g. adhesion between film and substrate, thermodynamics of displacive transformation and 
superelasticity effects [36]. Considering the curvature of the film-substrate system, the residual stress is classified as tensile or compressive, figure 2.17 (a) and (b) respectively.

(a)
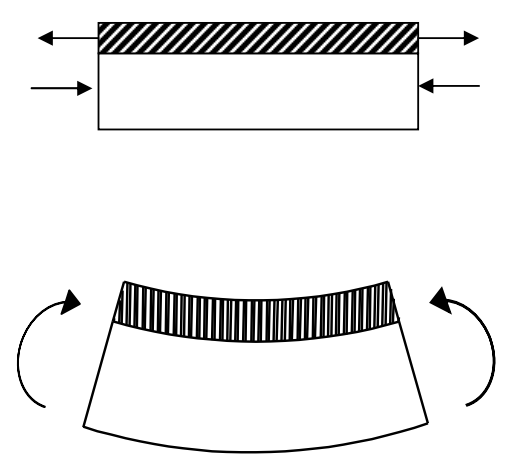

(b)
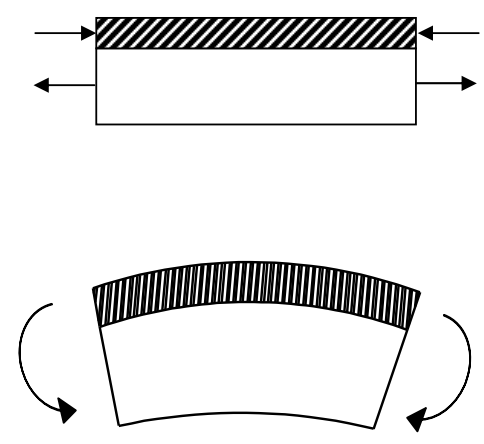

Figure 2.17: Residual stress classification: (a) residual tensile stress; (b) residual compressive stress in film.

Residual stress can be controlled by regulating the energetic degree of the bombardment. By using Stoney's equation [34] (equations 2.4, 2.5) derived by the principle shown in figure 2.18 ,

$$
\sigma=\frac{\gamma_{s} \cdot t_{s}^{2}}{6 \cdot\left(1-v_{s}\right) \cdot t_{f}} \cdot\left(\frac{1}{R}-\frac{1}{R_{o}}\right)
$$

the residual stress can be determined. Where $\gamma_{s}$ is the substrate Young's modulus, $t_{s}$ is the substrate thickness, $v_{s}$ is the substrate Poisson's ratio, $t_{f}$ is the film thickness. $R_{o}$ is the initial substrate curvature and $R$ is the final system curvature both given by

$$
R=\frac{L^{2}}{2 x}
$$

\section{Stoney's equation principle}

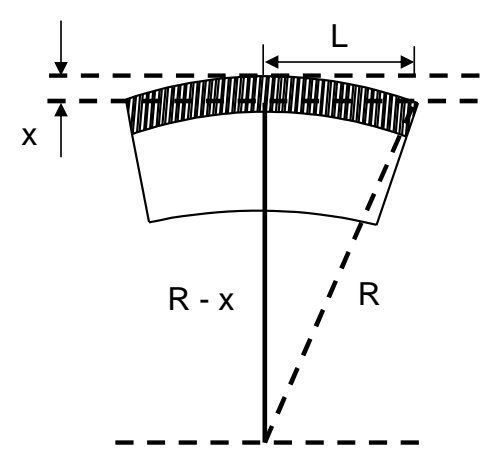

Figure 2.18: Residual stresses: Stoney principle. 


\subsubsection{UV lithography}

Lithography is based on the transfer of a desired pattern to a photoresist. The photoresist can be coated on the substrate through the use of spin, dip and spray coating. The selection of a suitable photoresist will be directly related to the desired lithography resolution, the related coating technique and the etching technique. For an enhanced resolution, electron beam lithography (EBL) might be used to deliver feature sizes smaller than $100 \mathrm{~nm}$ [37], using high energy electrons. Photolithography or UV lithography involve a set of steps, as summarized in Figure 2.19 .

(a)

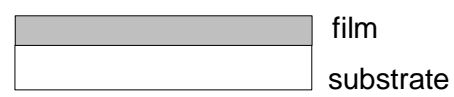

(b)

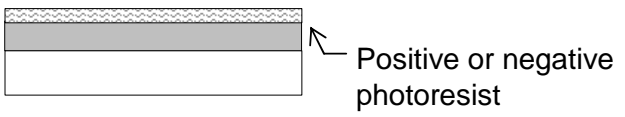

(c)

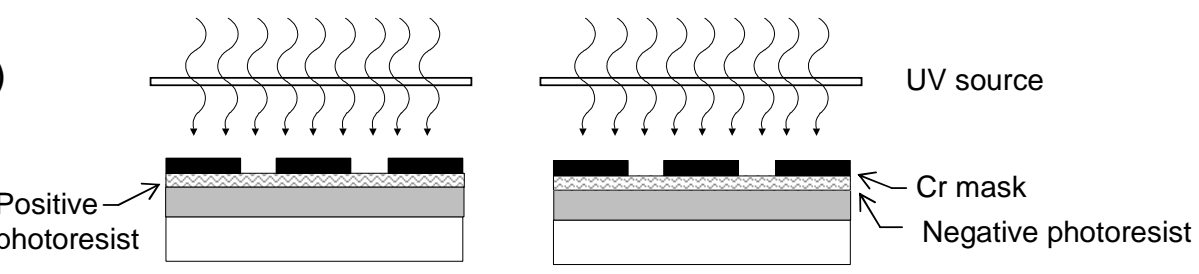

(d)
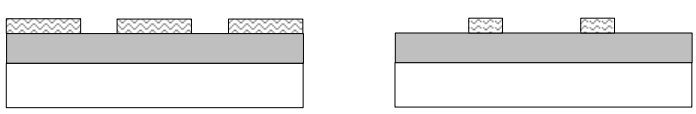

Figure 2.19: Summary of photolithography process steps: (a) thin film deposition (b) photoresist coat (spin, dip or spray coation) (c) UV exposure (d) photoresist development.

In the first step, the surface of the specimen is coated with a thin layer of an organic polymer (photoresist), figure 2.19 (b). Spin coating is used if the specimen to be coated is planar. The specimen is spun at high speeds, at these speeds centrifugal forces will act on the polymer pushing it homogeneously to the edge. The photoresist thickness $d$ is the function of the polymer viscosity and rotational speed of the specimen. If the 
specimen is not planar a dip coating method can be applied. The thickness of the dip coated photoresist, is a function of withdrawal speed, photoresist viscosity and sample geometry, as described by Landau, Levich and Derjaguin [38]. The quality of the spin and dip coating will define the density of defects transferred to the patterned device at the end of the photolithography process. After the coating process, $15 \%$ of the solvent is still present on the photoresist and it may contain stresses. Thus, soft bake is realized to reduce stresses, eliminate solvent concentration and promote a better adhesion of photoresist to the functional film. After softbake the film is exposed to UV light. Photoresist, as mentioned before, is an organic polymer which is sensitive to ultraviolet radiation and can be classified according to its sensitivity. With positive photoresists, figure 2.19 (c), the main and side chains of the polymer will be ruptured during the exposion, becoming more soluble. With negative photoresists, figure 2.19 (c), the main and side chains are cross-linked and the resist becomes less soluble. A Cr mask as shown in figure 2.19 (c) is usually a flat glass with an absorber desired $\mathrm{Cr}$ pattern. Photolithography can either be realized by contact mode, where the Cr mask is in contact with the photoresist or non-contact mode, where the $\mathrm{Cr}$ mask is slightly raised above the specimen. Contact mode is divided into soft and hard mode depending on whether the mask is pushed into more intimate contact to the specimen or not. The major disadvantage of the hard contact mode is the increase in the defect density due to the contact between the specimen and the Cr photomask. For this reason, contact mode is mainly limited to research and non-contact mode to mass production. The disadvantage of non-contact mode is the reduction in the resolution. Figure 2.20 shows intensity as function of the gap $(g)$ between mask and photoresist [39], considering a mask with single aperture $(W)$ and monochromatic light source exposion. When the gap is small such that

$$
\lambda<g<\frac{W^{2}}{\lambda}
$$

the system is in the near field region of Fresnel diffraction [40]. If this gap is very large, the image is severely degraded. Thus, we can define minimum feature size as 


$$
W_{\min } \approx \sqrt{k \cdot \lambda \cdot g}
$$

where $k$ is a constant depending on the photoresist process and typical values are close to 1. For example, using a non-contact mode gap of $20 \mu \mathrm{m}$ and a source wavelength of $436 \mathrm{~nm}$ the minimum feature size achieved is about $3.0 \mu \mathrm{m}$. Current state-of-the-art photolithography uses deep ultraviolet (DUV) light with wavelengths of 248 and $193 \mathrm{~nm}$, which allow minimum feature sizes down to $50 \mathrm{~nm}$ [41, 42].

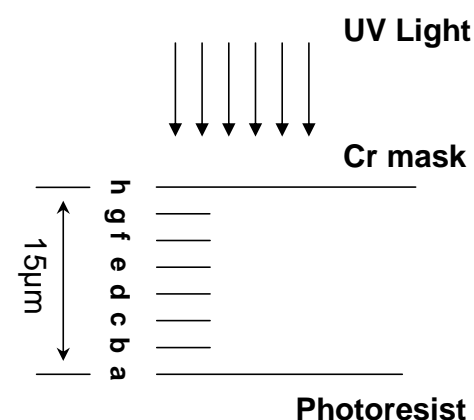

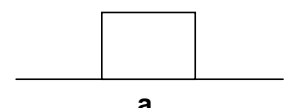
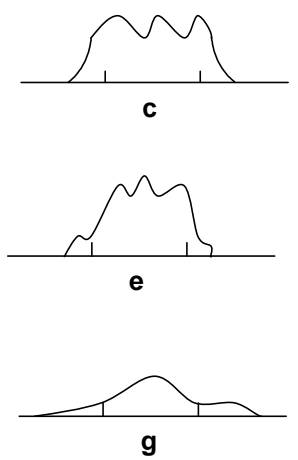
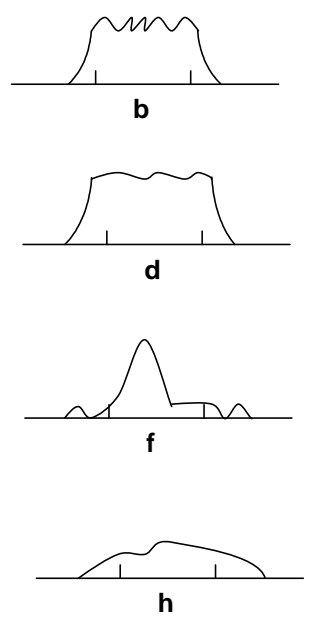

Figure 2.20: Intensity as function of the gap ( $g$ ) between mask and photoresist [39].

The exposure time (in seconds), is calculated by the division of incident energy $\left(\mathrm{J} / \mathrm{cm}^{2}\right)$ by incident light intensity $\left(\mathrm{W} / \mathrm{cm}^{2}\right)$. After exposure, the post bake is performed to complete the photoreaction initialized by exposure. The photoresist will be 
selectively dissolved during the development step, figure 2.19 (d). Hard bake must be realized to improve the resistance of the photoresist film. At this step, the photoresist is baked at high temperature $\left(120^{\circ} \mathrm{C}\right)$ and for longer periods (20 to 30 minutes).

\subsubsection{Etching process}

Etching is a process based on the transformation of solids into liquids or into a gaseous phase. Etching is classified into: wet chemical etching and dry etching. The wet chemical etching method is a process by which material is transformed from solid to liquid phase. The great advantage of wet etching is the high selectivity and the high etching rates. Selectivity is the ability to control which reaction will take place during the etching process. The great disadvantage is the isotropic etching behavior. Isotropic etching means that there is no etching preference of any direction and the etch rate is the same in all directions, as shown in figure 2.21 (a). An undercutting effect can be observed on the isotropic etching, i.e., material under the photoresist mask is etched. In this case, the horizontal etching rate $\left(\dot{E}_{h}\right)$ is the same as the vertical etching rate $\left(\dot{E}_{v}\right)$.

$$
\text { Isotropic etching }
$$

(a)
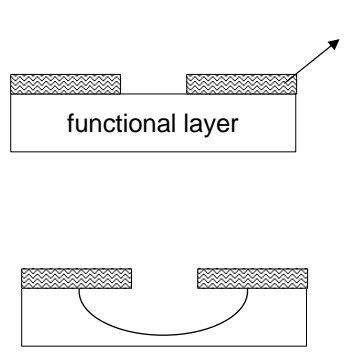

$\dot{\mathrm{E}}_{\mathrm{h}}=\dot{\mathrm{E}}_{\mathrm{v}}$

\section{Anisotropic etching}

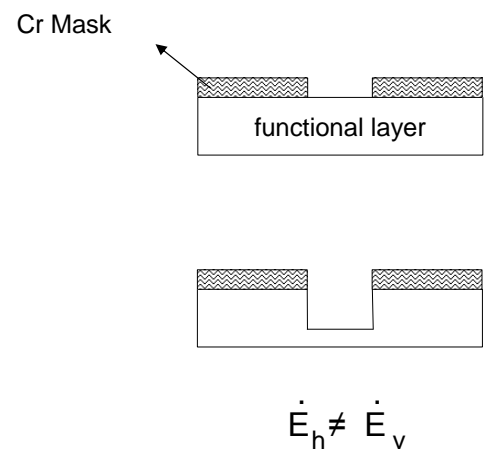

(b) 
the etchant and its reaction products. The etch rate $(\dot{E})$ is the main physical quantity for the etching process and is described by

$$
\dot{E}=\frac{\text { material(thickness) }}{\text { etching(time })}
$$

All etching methods by which material is transformed from solid to gas phase is called dry etching. Some types of dry etching are: plasma chemical etching, reactive ion etching sputtering, sputter etching, ion milling, ion beam assisted chemical etching and reactive ion beam etching [43]. In contrast to wet etching, dry etching methods show anisotropic etching behavior. Anisotropic etching means that the etching preference is restricted to one direction only, preferentially normal to the surface direction. This preference can be caused by: direction of attack or crystallographic properties of the material. Anisotropic etching shows no undercutting, i.e, the horizontal etching rate is different to the vertical etching rate, as shown in figure 2.21(b). The table 2.2 compares wet and dry etching methods [37, 43, 27].

Table 2.2: Comparison wet etching versus dry etching

\begin{tabular}{cll}
\hline & Wet etching & Dry etching \\
\hline Method & Chemical Solution & Ion Bombardment \\
\hline $\begin{array}{c}\text { Environment/ } \\
\text { equipment }\end{array}$ & Atmosphere, Bath & Vacuum Chamber \\
\hline Advantage & Low cost, easy to implement, & Capable of defining small \\
& high etching rate and good & feature size $(<100 \mathrm{~nm})$ \\
& selectivity for most materials & \\
\hline Disadvantage & Inadequate for feature & High cost, low etching rate \\
& size $<1$ um & and poor selectivity \\
\hline Directionality & Isotropic & Anisotropic \\
\hline
\end{tabular}




\subsection{Production and limitations of standard $\mathrm{TiNi}$ stents}

Superelastic shape memory materials are of special interest in medical applications due to the large obtainable strains, the constant stress level, fatigue resistance and their biocompatibility. Figure 2.22 summarizes the relevant properties of TiNi comparing relative limitation and body of knowledge.

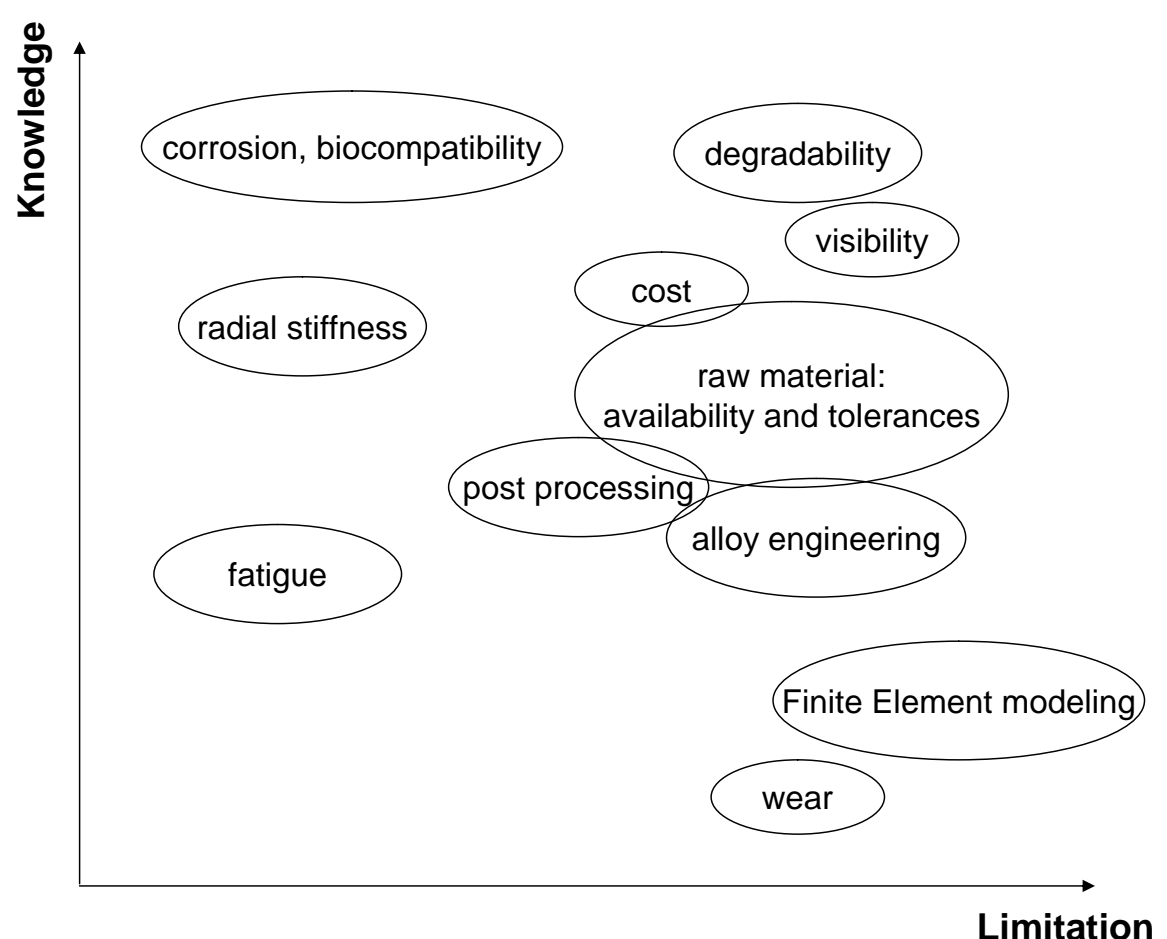

Figure 2.22: Summary of the relevant properties of TiNi with regard to their relative limitation and knowledge [1].

Some NiTi properties have been studied for years, but some concerns must still be addressed. In the case of corrosion and biocompatibility several studies have proved the good resistance of NiTi [44, but some points remain unclear and need to be improved, e.g the influence of the finishing method, galvanic coupling, mechanical damages and freetting and wear. The radial stiffness is a limitation for some applications, e.g calcified lesions, once TiNi stents elastic modulus varies from 28 to 83 GPa. Fatigue 
studies [45, 46, 47, 48] demostrate that NiTi devices are able to achieve the required cycles at strain amplitudes lower than $0.8 \%$. Due to NiTi special properties, e.g, nonlinear, hysteresis, strong temperature dependency, etc., Finite Element Modeling for $\mathrm{NiTi}$ is much more complex to model than a typical material. However, the constant improvement of modeling techniques does not turn this technique a limitation for medical applications. There are several NiTi compositions available for medical application, but some issues cannot be adressed e.g. production of ternary alloys to increase radiopacity. The fabrication of TiNi medical devices typically follows the process shown in figure 2.23 .

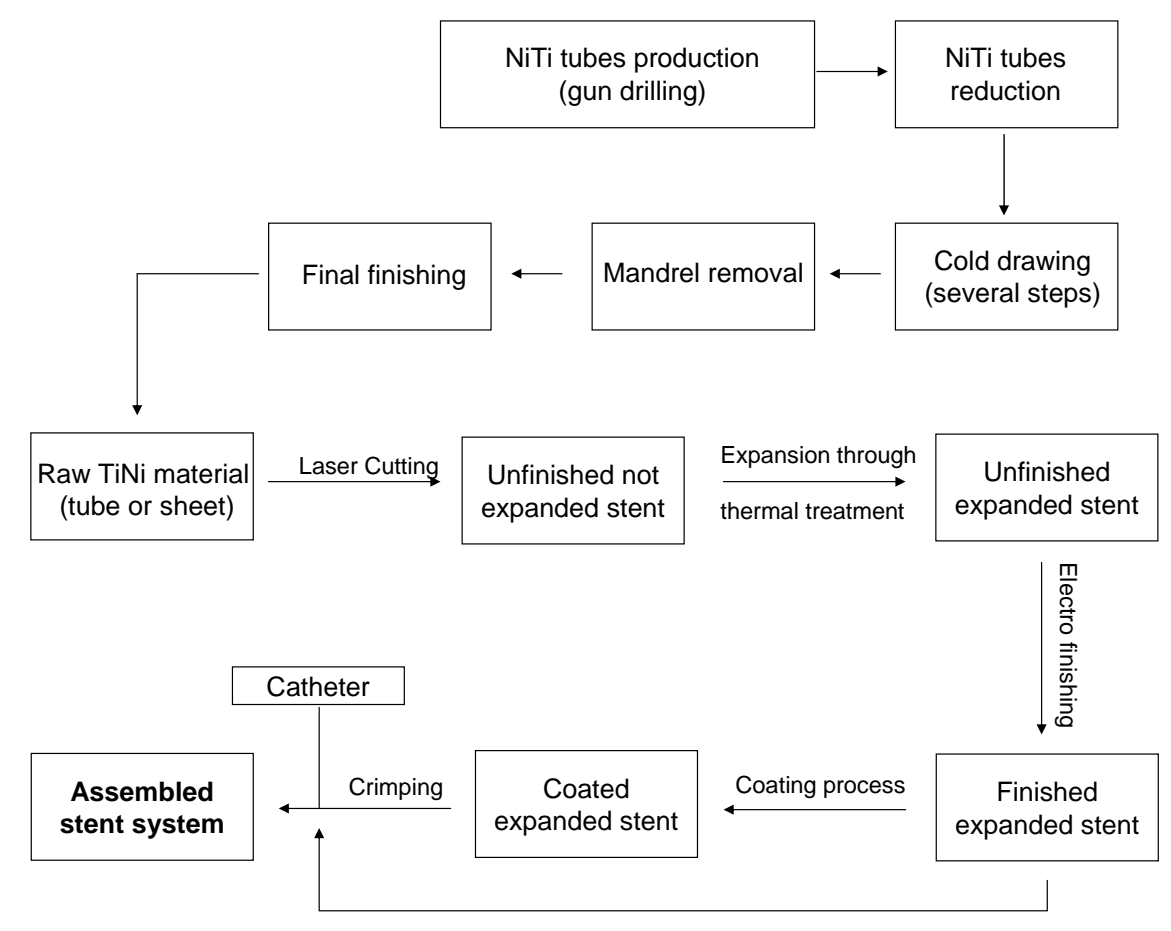

Figure 2.23: Standard stent production process flow.

TiNi stents production depends on TiNi raw material suppliers. There are several suppliers available in the market, but only a few are able to deliver TiNi tubes with the desired wall thickness tolerances, which will consequently reduce the tight wall tolerance of the finished device. Medical TiNi tubes with outer diameter in the range of 0.25-6 mm , generally, are reduced by a cold drawing process. Initially, the hollow NiTi 
tubes are prepared by gun drilling and have an outer diameter between 12.5-30 mm. To reduce the outer diameter to the desired dimension a cold drawing process is used. During cold drawing, a non-deformable or a deformable mandrel is used to maintain the internal tube diameter, as shown in figure 2.24 (a) and (b). For non-deformable mandrel process, a metal/alloy of low melting point is used as mandrel. After cold drawing, the mandrel is removed by melting. For deformable mandrel process the mandrel is constantly reduced with the tube diameter. After drawing and heat treatment the mandrel is then streched to a reduced diameter to facilitate its removal [49].
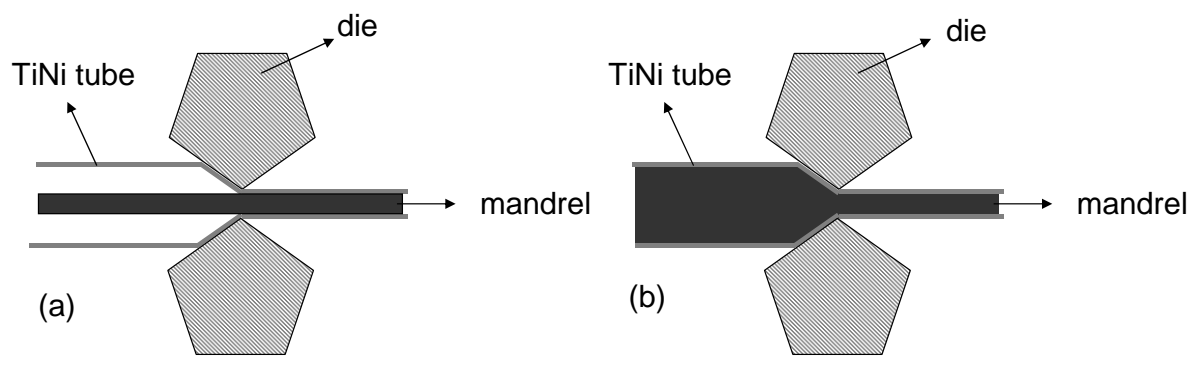

Figure 2.24: TiNi tube drawing process (a) non-deformable mandrel, (b) deformable mandrel.

The stent patterning is realized by high accuracy laser cutting and its tolerance is a few $\mu m$ depending on the laser source used in the process. The standard laser cutting aspect ratio, defined by the relation between wall thickness and cutting width, is 10:1 [9]. The interaction between laser and NiTi material generates a thermal effect which is responsible for the formation of the Heat-Affected Zone (HAZ) at the cutting edges. This zone is characterized by the formation of a resolidified layer of material which has different mechanical properties from the base material and contains high residual stresses. These two characteristics are responsible for a possible premature material failure [50]. After the laser cutting process, the stent is expanded to the desired internal diameter, using a pin process together with thermal treatment. Then, the stent is electro polished to improve the surface finish, enhance fatigue and corrosion resistance [51]. The stent can be coated with medication, depending on the application or be directly crimped into a catheter. Figure 2.25 shows two examples of stents prepared with the conventional 
technology by Admedes company.

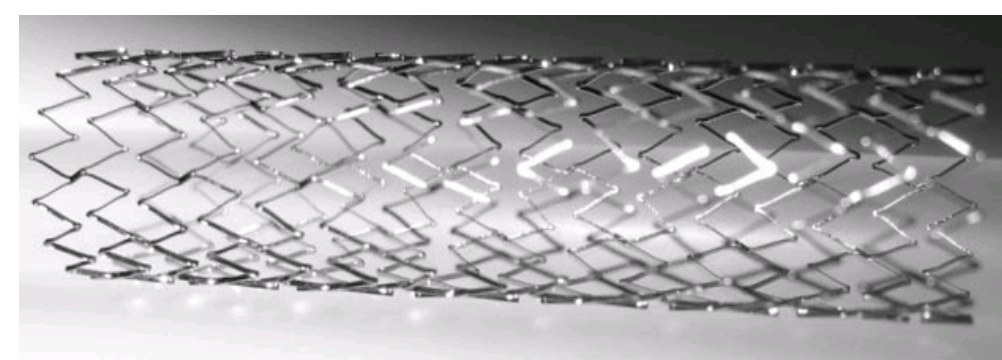

Figure 2.25: Example of stent device prepared with the conventional technology by Admedes company [Admedes Presse]. 


\subsection{State-of-art on cylindrical deposition/lithography}

Vapor deposition on cylindrical substrates are of great technological interest, i.e. coating of fibers with metallic alloys to create metal matrix composites [52], to synthesize solid state fiber batteries [53], filling of low resistivity metals for microeletronic interconnection [54], deposition of metal-aluminium nitride cermet on glass tubes as a solar absorbing layer [55], carbide coatings for cutting tools [56], coating of medical implants such as stents [57, 58], and decorative and functional coatings on complex shapes [59].

Several cylindrical deposition techniques have been developed for over 30 years. In 1955 Lipset and Faraday developed an apparatus to deposit gold thin film on cadmium single crystal of $1.0 \mathrm{~mm}$ diameter, as shown in figure 2.26. This development was based on cathodic sputtering, where a glass tube was used as a chamber. They achieved a homogeneous deposition rate of $300 \mathrm{~nm} / \mathrm{h}$ [60].

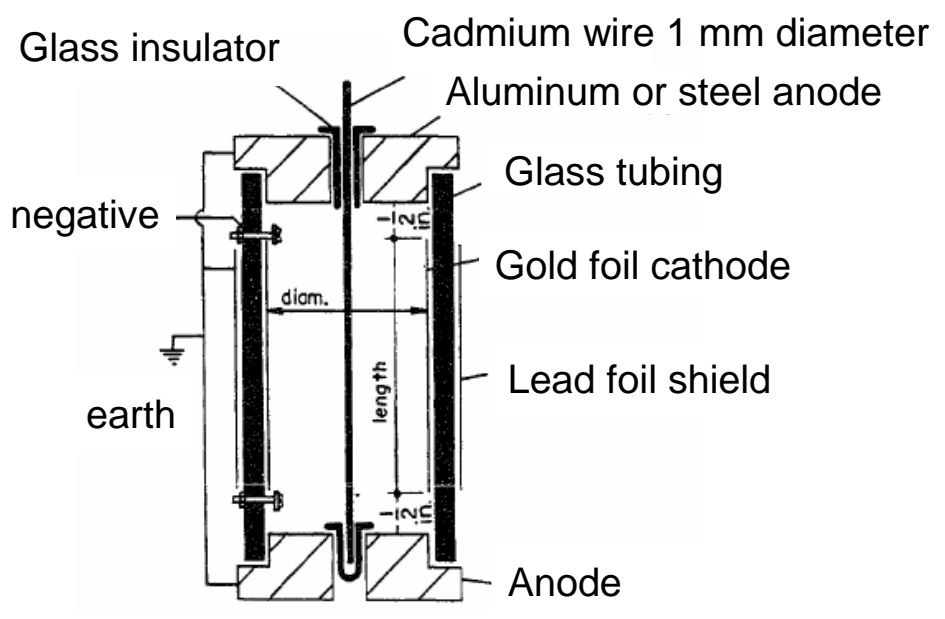

Figure 2.26: Lipset and Faraday arrangement of the sputtering chamber [60].

In 1974 Thornton and Hedgcoth [61] presented results obtained using hollow cathode sputtering, wherein substrates were mounted on the axis on an tubular source, providing a solution for complex shape deposition, as shown in figure 2.27. A lot of effort was invested in the development of cylindrical deposition methods for industrial production, where target utilization, uniformity growth of the films, process stability and efficiency 
were taken in consideration.

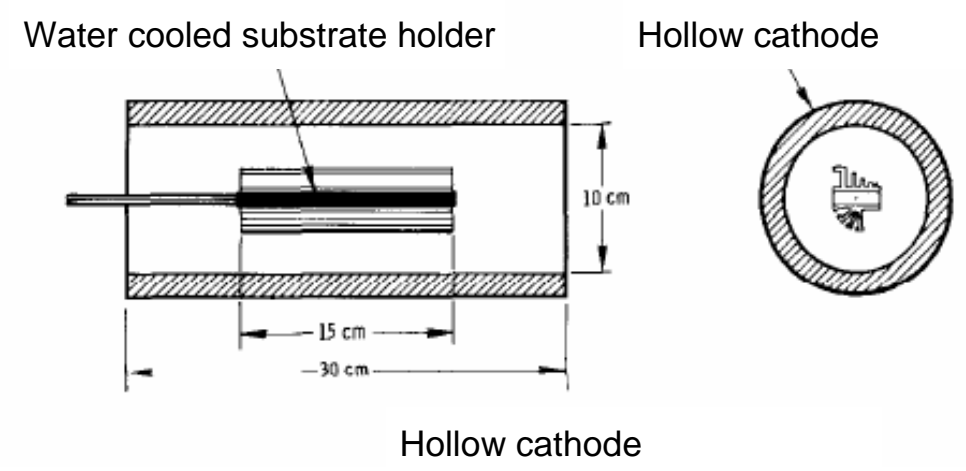

Figure 2.27: Hollow cathode setup by Thornton and Hedgcoth [61] .

Siegfried et al. [59], have demostrated how the hollow cathode technique can be applied in large substrates, using a cathode with outside diameter $19 \mathrm{~cm}$ and length 21 $\mathrm{cm}$, as shown in figure 2.28 . They estimated and compared the cost for a typical drill bit coated with TiN using planar magnetron and the cost of the same drill bit coated with cylindrical magnetron. They concluded that the use of cylindrical magnetron reduced the material cost by a factor of five and additionally provided an uniform coating on large samples without substrate rotation.

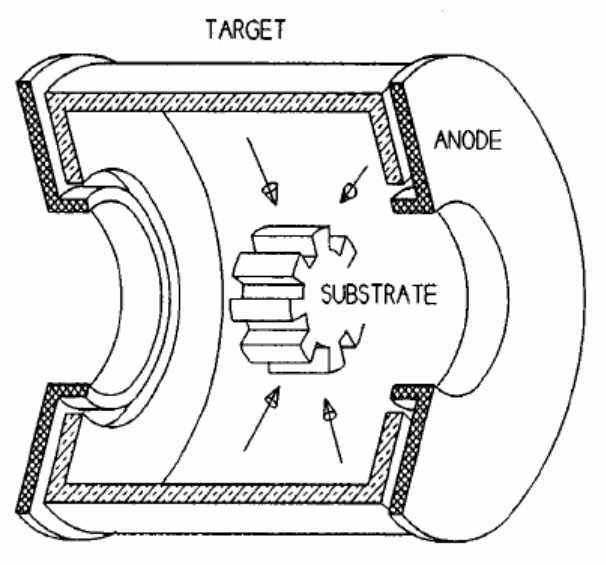

Figure 2.28: Hollow cathode setup applied in large substrates by Siegfried et al [59]. 
A number of advanced PVD methods for metalcutting are available. Depending on the intended application (monolayer or multilayer) different PVD technologies are used, i.e. electron beam evaporation, cylindrical sputtering and arc evaporation. Ward-Close and Partridge [62] described a fiber coating process based on an electro beam evaporation technique for coating $100 \mu \mathrm{m}$ diameter SiC fiber, as shown in figure 2.29. A $10 \mathrm{~kW}$ electron-beam heated double evaporation source from the company Temescal was used, the fibers were rotated at $300 \mathrm{rpm}$ and the deposition rate was approximately 5 to 10 $\mu \mathrm{m} /$ minute.

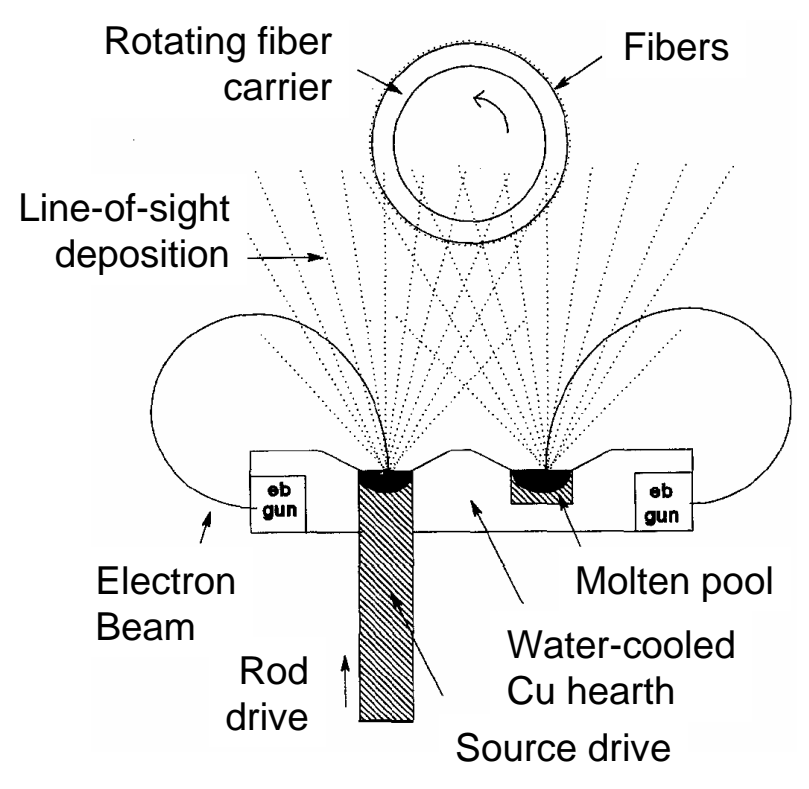

Figure 2.29: Electron-beam heated double evaporation source setup by Ward-Close and Partridge [62].

Chemical Vapor Deposition (CVD) allows uniform coating thickness on a fiber due to the flux being uniformly distributed over the sample surface. However, this method required the use of an expensive precursor material and the depositon of metallic alloys is very difficult. Hass et al. 63] studied an approach called Direct Vapor Deposition (DVD), as shown in figure 2.30. They used a continuos electron-beam in a low vacuum environment to deposit aluminium on a $380 \mu \mathrm{m}$ diameter, $13 \mathrm{~cm}$ long stainless steel fiber with a deposition rate of approximately $3 \mu \mathrm{m} /$ minute. They studied the influence of the kinetic energy of the gas jet and vapor atoms on the thickness uniformity around 
the fiber surface, conlcuding that low kinetic energy of the vapor atoms led to a high uniformity.

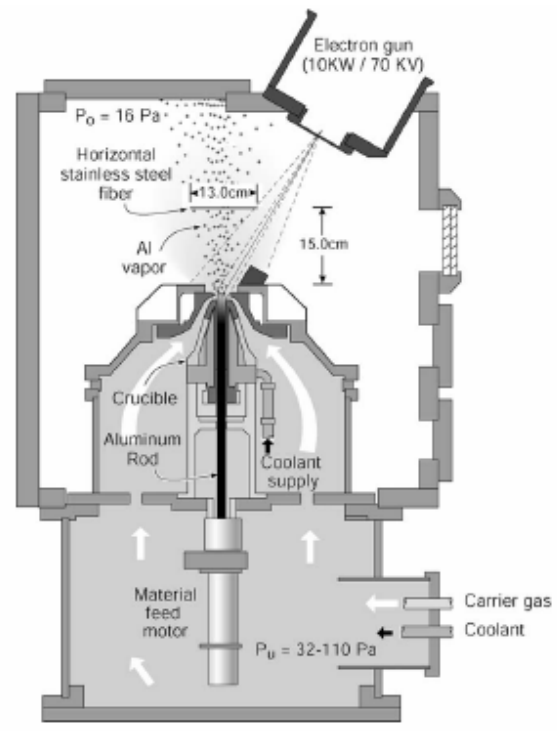

Figure 2.30: Direct vapor deposition coating system by Hass et al [63].

Ducros et al. 64] described a novel method to deposit $\mathrm{CrN} / \mathrm{TiN}$ and TiN/AlTiN multilayer on cemented carbide cutting tools by means of arc evaporation in order to improve flank wear and cutting time. An industrial size cathodic arc evaporation device equipped with four targets is used to deposit such multilayers. This device is equipped with a threefold rotating substrate holder with variable rotation speed. The deposition rate achieved varies between 1 to $2 \mu \mathrm{m}$ /hour.

Zhang et al. [55] presented a novel method to coat glass tubes with metal-aluminium nitride for solar collector tubes by means of commercial scale direct current magnetron sputtering system, where the vacuum chamber of $1.8 \mathrm{~m}$ high and an inner diameter of $0.8 \mathrm{~m}$ was equipped with 3 cylindrical targets of $1.5 \mathrm{~m}$ long and $37 \mathrm{~mm}$ diameter, as shown in figure 2.32 . The targets were separated by a screen to prevent contamination. During the sputtering the permanent magnet is rotated in order to ensure uniform target erosion.

Two groups in Japan (Buenconsejo et al. [65] and Komatsubara et al. [66]) have 


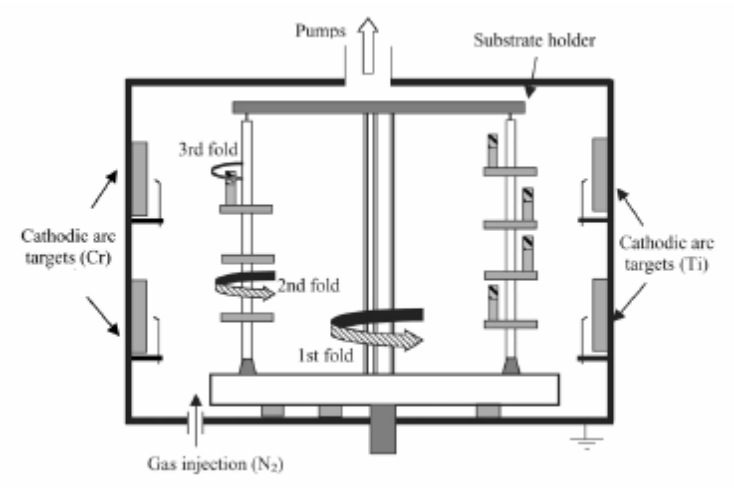

Figure 2.31: Industrial size cathodic arc evaporation device equipped with four targets by Ducros et al 64].

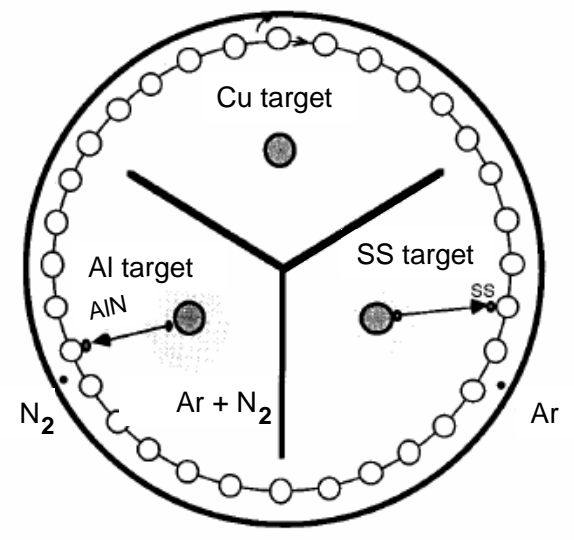

Figure 2.32: Industrial size direct current magnetron sputtering system by Zhang et al [55].

used planar magnetron sputtering to sputter TiNi microtubes. They developed, as also developed in this dissertation, a rotational device which is mounted in a vacumm chamber and provides movement to the substrate. The work presented by Komatsubara et al. is a description of the rotational device, they did not test the properties of the sputtered TiNi cylindrical films. Buenconsejo et al. deposited $6 \mu \mathrm{m}$ TiNi on a $50 \mu \mathrm{m}$ $\mathrm{Cu}$ substrate. They studied the influence of the substrate speed on the film growth, concluding that microtubes fabricated under higher speed (15 and $30 \mathrm{rpm}$ ) exhibited high fracture stress and a maximum $3 \%$ strain.

Cylindrical lithography, in comparison to cylindrical sputtering is not very well 
developped. S. Snow et al. [67, 68] presented a novel method of structuring sputtered fibers of $55 \mu \mathrm{m}$ diameter by means of laser and electron beam, as shown in figure 2.33 .

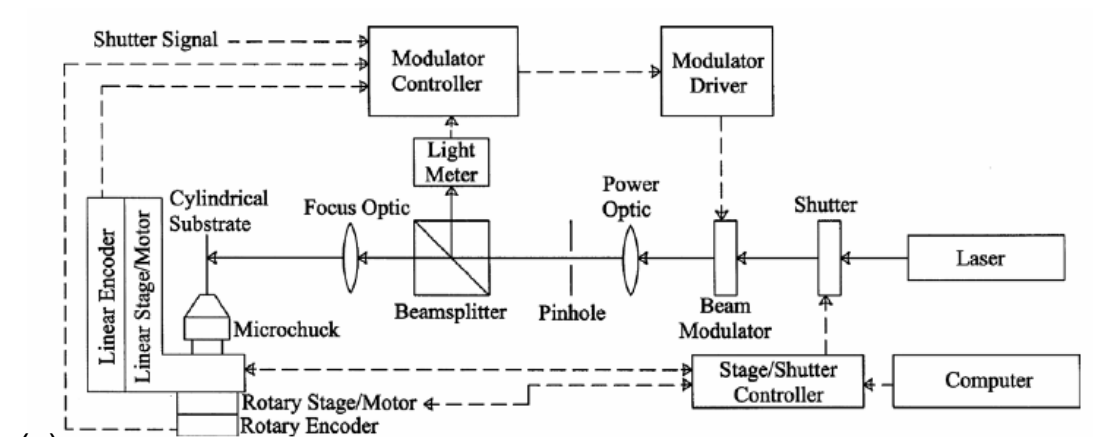

(a)

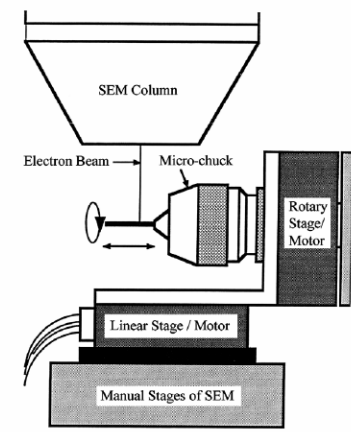

(b)

Figure 2.33: Cylindrical lithography presented by S.Sow et al. [67], [68]

(a) with laser exposure (b) with electron beam.

Throughout this dissertation, a new cylidrical lithography method based on UV lithography and synchronization of the planar Cr mask with the rotation of the cylindrical substrate has been developed and published [69. Details will be presented in section 4.3 .1 . 


\section{Chapter 3}

\section{Experimental Techniques}

During this dissertation several standards of experimental techniques were used. This chapter will approach and briefly describe the standard experimental characterization techniques, as well as three fabrication techniques: magnetron sputtering, UV lithography and rapid thermal annealing.

\subsection{Standard characterization techniques}

\subsubsection{Scanning Electron Microscope - SEM and Energy Dispersive X-ray Spectroscopy - EDX}

As mentioned in section 2.3, the $\mathrm{Ti}$ and $\mathrm{Ni}$ solubility in the TiNi phase is very narrow causing high property dependence of the compositon. Thus, composition of as-deposited amorphous films was determined by Energy Dispersive X-ray Spectroscopy (EDX: Oxford instruments INCA 3.04) within a scanning electron microscope (SEM FEI - Helius), which was also used to analyse surfaces, as well as breaking edges of a sample after tensile and fatigue tests. A precise standard $T i_{50} N i_{50}$ at $\%$ was used as reference material for all TiNi stoichiometry characterization. In this method, the sample is excited by an incident electron creating a hole in the $\mathrm{K}$ shell, this hole is filled by an electron from an outer shell, which lowers its energy. X-ray is then emmited. $K_{\alpha}$ is produced if the 
electron transition occurs between $\mathrm{L}$ and $\mathrm{K}$ shells and $K_{\beta}$ from $\mathrm{M}$ to $\mathrm{K}$. The difference between the involved levels is the X-ray energy or wavelength and each material in the periodic table has its own characteristic X-ray energies. The lateral and vertical resolution of the technique is approximately $1 \mu \mathrm{m}$.

\subsubsection{Differential Scanning Calorimetry - DSC}

This method can be defined as a thermoanalytical method in which the difference in heatflux between the sample and the reference are measured as a function of temperature and was used to measure the phase transformation temperature in TiNi shape memory alloys. The DSC is illustrated schematically in figure 3.1. The sample is prepared in such a way that it is in good contact with the furnace. The sample pan is sealed and placed in one side of the holders, in the other side an inert reference material is placed.

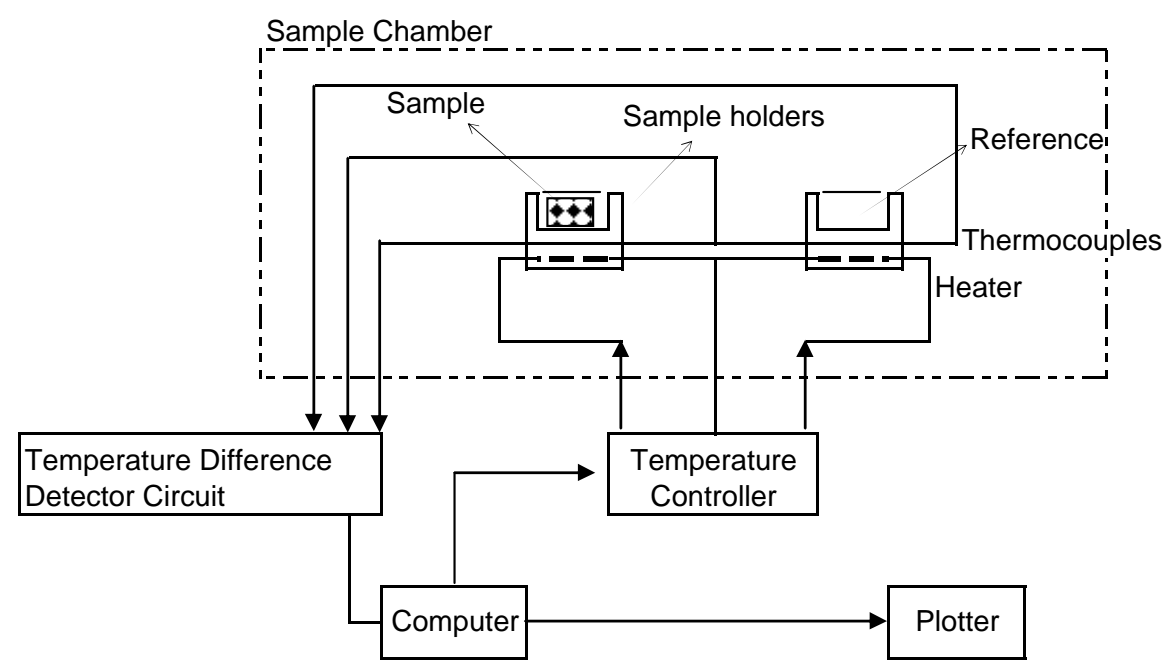

Figure 3.1: Schematic diagram of DSC device.

Thermocouples placed at the base of each side of the holder will measure the temperature difference between sample and reference material as a function of the furnace temperature. The temperature of the furnace and the heat sensitive plate is also measured by a second series of thermocouples [70]. Figure 3.2 shows a schematic DSC curve of a TiNi thin film shape memory alloy. When the martensitic transformation takes place, 
numerous physical properties are changed. During the transformation, latent heat associated with the transformation is absorbed or released depending on the transformation direction. The forward, austenite-to-martensite transformation is accompanied by the release of heat corresponding to a change in the transformation enthalpy (exothermic phase transformation). The reverse, martensite-to-austenite transformation is an endothermic phase transformation accompanied by absorption of thermal energy. For a given temperature, the amount of heat is proportional to the volume fraction of the transformed material.

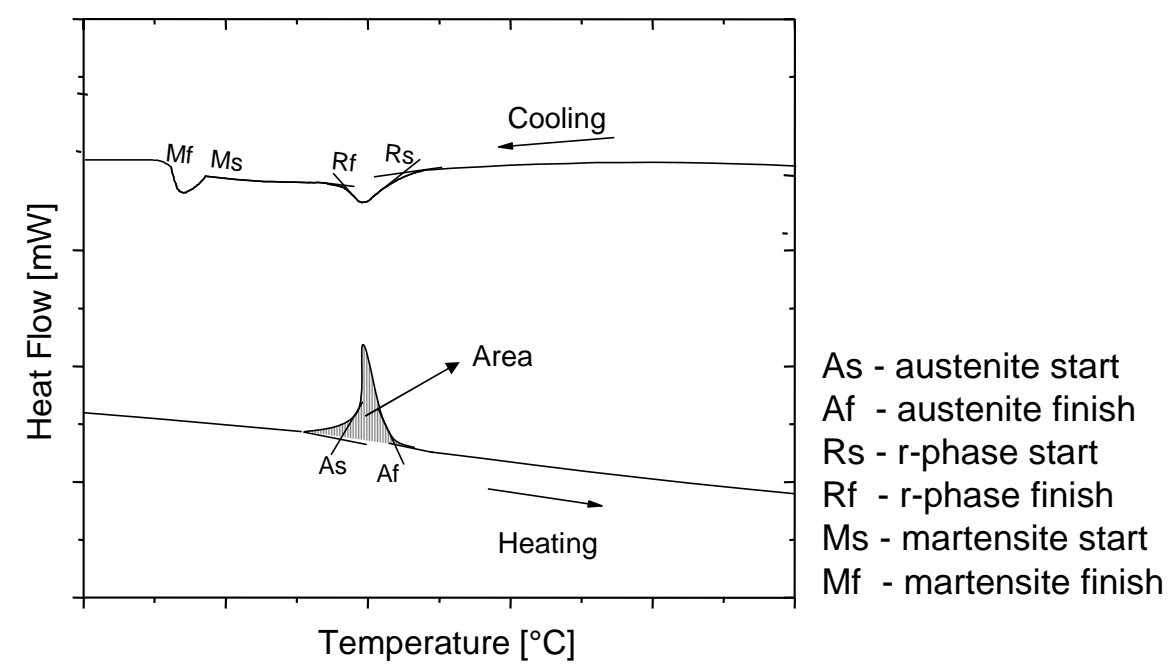

Figure 3.2: Schematic heat flow curve of a TiNi shape memory alloy.

A DSC device from the company Perkin Elmer was used. Differential scanning calorimetry data were recorded between -60 and $150{ }^{\circ} \mathrm{C}$ with a typical rate of $10 \mathrm{~K} / \mathrm{min}$. In order to start the DSC measurements at a well-defined state, samples were heated well above the formation temperature of austenite $\left(\mathrm{T}>A_{F}\right)$ and kept at a temperature of $150{ }^{\circ} \mathrm{C}$ for 3 min before starting the measurement.

\subsubsection{X-ray Diffraction - XRD}

X-ray diffraction was employed to determine the crystalline structure of the sputtered/annealead TiNi thin film. A Seifert XRD 3003 X-ray diffrectrometer was used. Figure 3.3 shows 
the data used on XRD analysis of TiNi thin films.

\begin{tabular}{|c|c|c|c|}
\hline \multicolumn{4}{|c|}{ TiNi - Austenite 18-899* } \\
\hline$d h k l$ & $I$ & $2 \theta($ CuK $\alpha)$ & $h k l$ \\
\hline 2.111 & 100 & 42.3 & 110 \\
\hline 1.222 & 60 & 78.2 & 211 \\
\hline 1.496 & 40 & 62.0 & 200 \\
\hline
\end{tabular}

\begin{tabular}{|c|c|c|c|}
\hline \multicolumn{4}{|c|}{ TiNi - R-phase (+) } \\
\hline$d h k l$ & $I$ & $2 \theta($ CuK $\alpha)$ & $h k l$ \\
\hline 2.11 & & 42.82 & 110 \\
\hline 2.13 & & 42.35 & 211 \\
\hline
\end{tabular}

\begin{tabular}{|c|c|c|c|}
\hline \multicolumn{4}{|c|}{ Ti2Ni 18-898* $^{*}$} \\
\hline$d h k l$ & $l$ & $2 \theta($ CuK $\alpha)$ & $h k l$ \\
\hline 2.171 & 100 & 41.563 & 511 \\
\hline 2.302 & 30 & 39.098 & 422 \\
\hline 1.994 & 30 & 45.449 & 440 \\
\hline 1.329 & 25 & 70.844 & 822 \\
\hline 1.133 & 25 & 85.665 & $933 / 711$ \\
\hline
\end{tabular}

\begin{tabular}{|c|c|c|c|}
\hline \multicolumn{4}{|c|}{ TiNi - Martensite 35-1281* } \\
\hline$d h k l$ & $I$ & $2 \theta($ CuK $\alpha)$ & $h k l$ \\
\hline 2.181 & 100 & 41.364 & $1-11$ \\
\hline 2.016 & 94 & 44.926 & 111 \\
\hline 2.295 & 54 & 39.222 & 020 \\
\hline 2.06 & 54 & 43.915 & 002 \\
\hline 2.005 & 26 & 45.186 & 021 \\
\hline 1.533 & 26 & 60.327 & 022 \\
\hline
\end{tabular}

\begin{tabular}{|c|c|c|c|}
\hline \multicolumn{4}{|c|}{ Ti3Ni4 - 39-1113* $^{*}$} \\
\hline$d h k l$ & $I$ & $2 \theta($ CuK $\alpha)$ & $h k l$ \\
\hline 2.092 & 100 & 43.21 & 122 \\
\hline 2.388 & 30 & 37.636 & 131 \\
\hline 1.845 & 20 & 49.353 & 312 \\
\hline 1.489 & 20 & 62.304 & 422 \\
\hline 1.22 & 20 & 78.304 & 532 \\
\hline 2.245 & 15 & 40.133 & 202 \\
\hline 1.675 & 15 & 54.757 & 232 \\
\hline
\end{tabular}

* from Powder diffraction file database (1994)

(+) Hwang et al., Stressed in situ X-ray diffraction Studies of a NiTi shape memory alloy, Mat. Res.

Soc. Symp. Proc. Vol.276 (1992) 183-188

Figure 3.3: Data used on XRD analysis of TiNi thin films.

This technique uses X-rays of known wavelength in order to determine the unknown spacing crystal plane. Monochromatic radiation is used and measurements can be performed either on single crystals or polycrystalline specimens.

The crystalline structure of the measured thin film on this work were measured based on the Bragg-method, where the sample is placed in such a way that the reflecting planes make an angle $\theta$ with the incident beam and the detector is set at the angle $2 \theta$, as shown in figure 3.4 . 


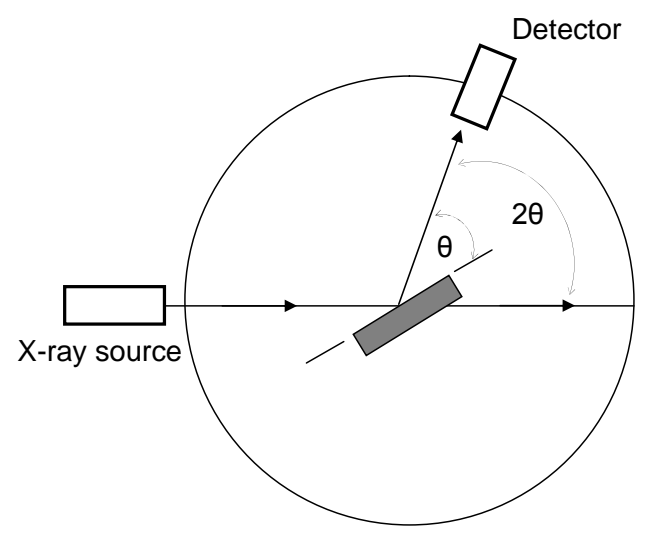

Figure 3.4: Schematic Bragg-method.

\subsubsection{Transmission Electron Microscopy - TEM}

TiNi thin film microstructures were analysed: as sputtered and after heat treatment by using a transmission electron microscope Philips CM 30. The TEM samples were prepared by means of Focuses Ion Beam technology (FIB) installed in a FEI scanning electron microscope - Helius.

Transmission electron microscopy uses high electron energy to irradiate very thin specimens of around $0.1 \mu \mathrm{m}$ or less in thickness. The electrons are transmitted and form a specimen image of the specimen magnified in several steps with the aid of electronoptical lenses. The entire image can be observed directly on the fluorescent screen. The electrons emitted from the electron gun are acelerated to an energy up to $100 \mathrm{keV}$. The condenser lens are reponsible to control the beam emitted shape. The objective lens produces a first image, which is then further magnified by the intermediate and projector lens and finally projected onto the fluorescent screen. The image contrast is generated in the following way: the incident electrons are elastically scatered or diffracted in the specimen by small angles. Only those electrons which penetrate the specimen without scattering are used for image formation. Figure 3.5 shows the TEM imaging modes. In bright-field (BF) imaging, 3.5 (a), a small apperture is employed and centered onto the optical axis so that in effect only the directly transmitted beam pass through. In the dark-field (DF) images, 3.5 (b), the image is generated not by the direct beam but by 
the intensity of scattered electrons [71].

(a)

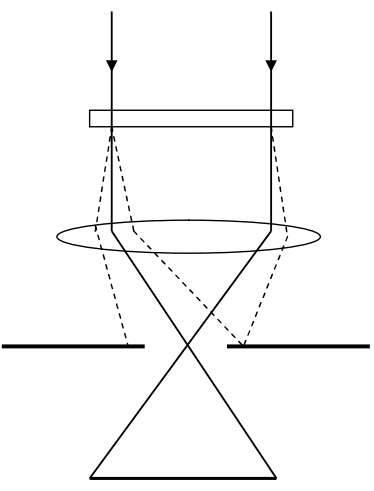

specimen

objective lens

objective aperture

first image (b)

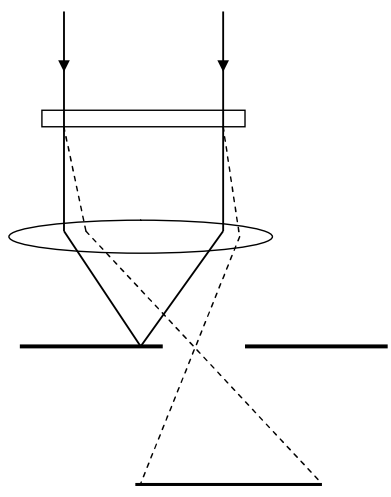

Figure 3.5: TEM imaging modes: (a) bright-field (BF) imaging, (b) darkfield (DF) images.

\subsubsection{Profilometer}

Thin film depostion rate, as well as the etching rate and photoresist thickness were controlled by an Ambios profilometer XP-2. The principle of this method is called Stylus, and consists of mechanical contact between the sample and a needle. The difference in height, between sample and needle, is measured and a step height profile is generated. This equipment is equipped with a motorized stage, a vacuum chuck, and its step height resolution is $10 \AA$.

\subsubsection{Tensile test}

Tensile test is the most commonly used mechanical test. A specimen is extended under a uniaxial tensile force until the separation occurs. The force required to deform the specimen is recorded as a function of extension and from these data a number of intrisic properties maybe derived, e.g. modulus of elasticity, yield and ultimate tensile strengths, ductility and critical stress on martensite transformation.

Tensile testing of freestanding films was performed in an universal tensile test device (Messphysik UTM Beta EDC 100N) with the sample being located within a temperature 
chamber (Shimadzu TCL-N382). To measure superelaticity the temperature must be set above $A_{s}$ temperature, as described in section 2.1.3. A pre-load of $0.2 \mathrm{~N}$ is applied in order to assure that the film is pre-stretched and well fixed. A constant strain speed of $0.4 \% / \mathrm{min}$ is applied.

\subsection{Fabrication techniques}

\subsubsection{Magnetron sputtering}

An Alcatel 450 magnetron sputtering device was used to produce TiNi thin films. This device is equipped with a DC $(600 \mathrm{~W})$ and $\mathrm{RF}(600 \mathrm{~W})$ magnetron power supply. The system consists of a main chamber with three $100 \mathrm{~mm}$ diameter cathodes and a load lock. The substrate holder is equipped with a heater, which reaches a maximum temperature of $500{ }^{\circ} \mathrm{C}$. The base pressure of the vacuum chamber is approximately $1 \times 10^{-7}$ mbar. The distance between sample holder and cathode can be regulated between $4.5 \mathrm{~cm}$ and $10 \mathrm{~cm}$.

\subsubsection{UV lithography}

UV exposure was performed with a Karl Suss mask aligner (MJB4). The device is equipped with a near UV Hg lamp of $200 \mathrm{~W}$ which generates an uniform intensity of $28 \mathrm{~mW} / \mathrm{cm}^{2}$ over an area of $100 \mathrm{~mm}$ (diameter). The intensity was measured using an intensity meter Model 1000 (Suss. MicroTec, Germany).

\subsubsection{Rapid Thermal Annealing - RTA}

The heat treatment was carried out ex situ by means of a rapid thermal annealing (RTA) system from the Createc company. The halogen lamp driven heating chamber enables typical heating ramps of $50 \mathrm{~K} / \mathrm{s}$ in a vacuum environment of about $10^{-6}$ to $10^{-7} \mathrm{mbar}$. The annealing temperature time and temperature steps were varied to understand their influence on the film crystallization, as well as on its mechanical properties. 


\section{Chapter 4}

\section{Results and Discussion}

\subsection{Fabrication and characterization of planar thin films}

\subsubsection{Sputtering device characterization}

As mentioned in section 3.2.1 an Alcatel 450 magnetron sputtering device was used to produce thin films. The deposition homogeneity and deposition rate along a 4 inch silicon wafer was analyzed as a function of the sputtering mode (RF or DC), power and distance between target (cathode) and substrate. The Si wafer 4 inch substrate was prepared before the deposition with ten Si pieces $(5 \times 5 \mathrm{~mm})$ distributed every $12 \mathrm{~mm}$ along the $\mathrm{x}$ and $\mathrm{y}$ axis. These pieces were used as a mask and after the deposition the steps created between deposited thin film and Si substrates in both directions were measured using a profilometer (see section 3.1.5).

At first, the deposition homogeneity from RF and DC sputtering process was analyzed and compared, as shown in figure 4.1 (a). A "quadratic function" on the film thickness distribution can be observed, where the maximum is in the middle of the 4 inch Si wafer substrate for both processes and corresponds to the point zero on the graphic $\mathrm{x}$-axis. The distribution of the film thickness using the RF sputtering process is smoother than the distribution achieved by the DC sputtering process. 

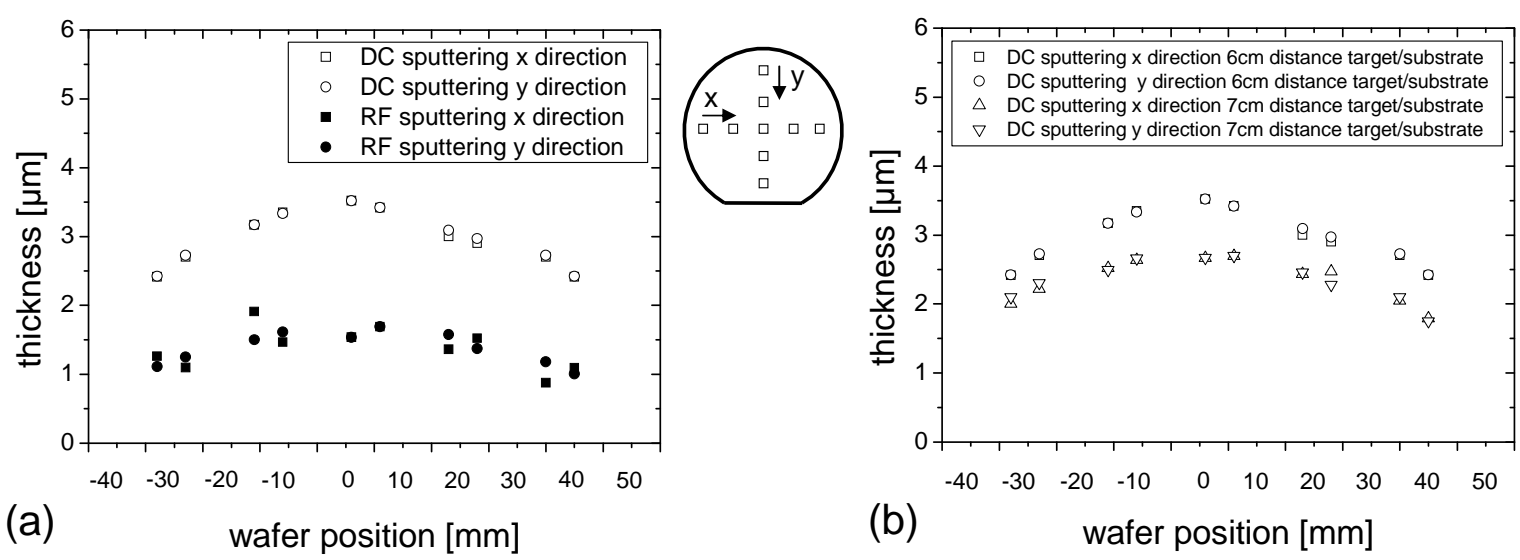

Figure 4.1: (a) Comparison of deposition homogeneity between RF and $D C$ sputtering processes on a 4 inch Si wafer, (b) Influence of the distance between target and substrate on the deposition homogeneity. Sputtering parameters: target used $\mathrm{Ti}_{53.2} N i_{46.8}$ at \%, Power $=500 \mathrm{~W}$, vacuum pressure $=2.3 \times 10^{-3}$ mbar, Ar flow $=20 \mathrm{sccm}$, time $=15$ minutes and distance between target and substrate $=6 \mathrm{~cm}$.

To investigate the influence of the distance between target and substrate on the deposition homogeneity, two sets of Si wafers were deposited with various distance between target and substrate. Figure 4.1 (b) shows that the wafer deposited at a large distance has a smooth distribution on the film thickness.

The observed film thickness distribution matches the function described by Soloviev et al. [32], which depends on the chamber geometry and the magnetic field, see section 2.4.1. The magnet ring placed above the target, as explained in section 2.4.1, is responsible for the increase in the ionization rate by further increasing the emitted secondary electron rate. Due to this magnet ring an irregular/circular material loss on the target is observed, forming a sputtering trench, as shown in figure 4.2. The irregular material loss is responsible for waste of more than $80 \%$ of a target and also for the irregular deposition distribution, shown in figure 4.1 (b). To reduce the irregular deposition thickness, the substrate must be placed as far as possible from the target. The Alcatel device is equipped with a movable substrate table which allows the adjustment of the distance between target and substrate, from 10 to $4.5 \mathrm{~cm}$. 


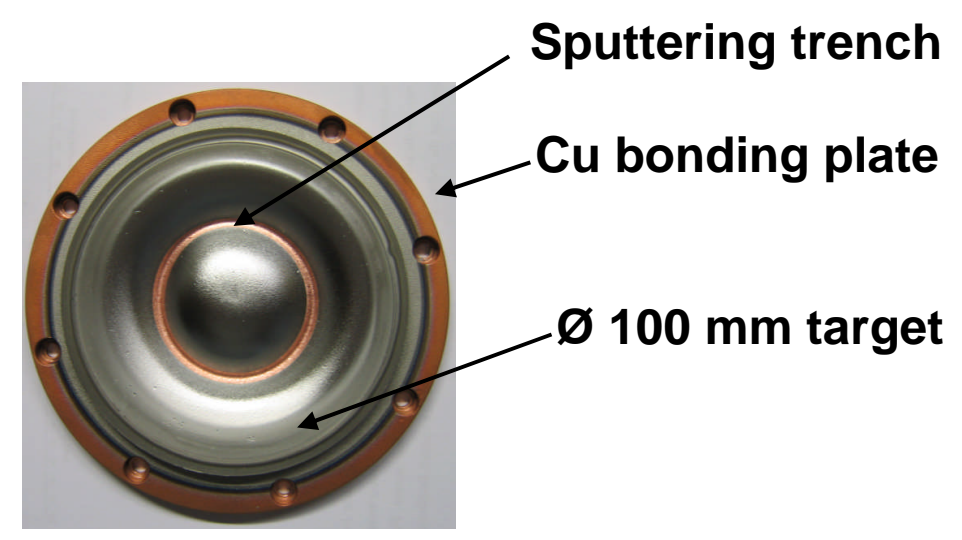

Figure 4.2: Irregular/circular material loss on the magnetron sputtering target.

Deposition rates of a $T i_{53.2} N i_{46.8}$ at $\%$ target as a function of two different magnetron power supplies (RF and DC), power and distance between target and substrate were analyzed.
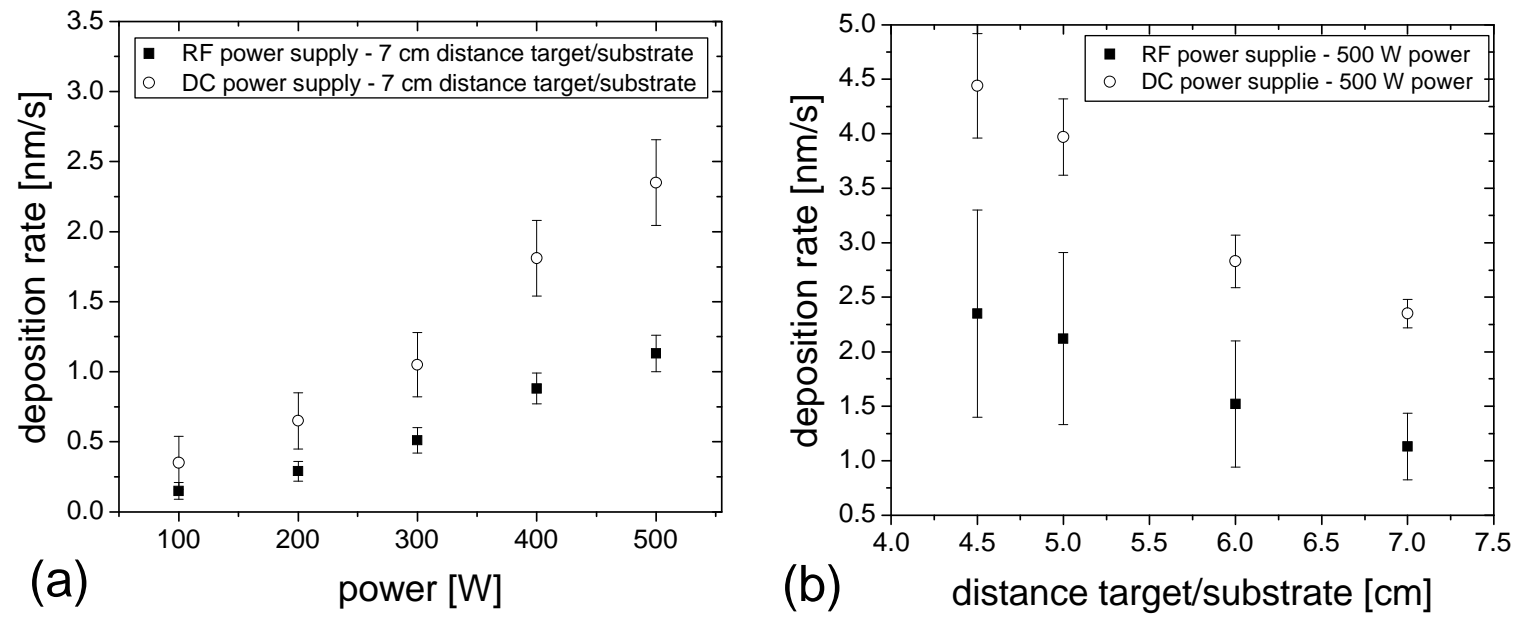

Figure 4.3: (a) Comparison of deposition rate of a $T i_{53.2} N i_{46.8}$ at $\%$ target as a function of applied power supply (RF and DC) (b)Influence of the distance target/substrate on the deposition rate. Vacuum pressure and Ar flow were identical in all experiments: $20 \mathrm{sccm}$ Ar and $2.3 \times 10^{-3}$ mbar.

Figure 4.3 (a) shows an increase of the deposition rate average with enhancement of the applied power, while the distance between substrate and target was kept constant. 
The DC power supply shows an average deposition rate two times higher than the RF power supply. The error bars correspond to the standard deviation from the homogeneity deposition results. Figure 4.3 (b) shows the decrease of the deposition rate with the reduction of the distance between target and substrate, while the power was kept constant. With a higher power, the ionization of argon is enhanced, leading to more ions bombarding the target. Thus, the kinetic energy of the ions increases resulting in a higher deposition rate. TiNi targets used during this research were manufactured by cast melting at the Mechanical Engineering Department of the University of Bochum [72. Table 4.1 lists the deposition rate of the materials used in this research and their deposition parameters.

Table 4.1: Planar deposition rate list of the materials used in this work and their deposition parameters

Deposition Parameters

\begin{tabular}{ccccccc}
\hline & & & \multicolumn{5}{c}{ Deposition Parameters } \\
\cline { 5 - 7 } Target & Deposition & Deposition & & & & \\
& rate DC & rate RF & Power & Pressure & Ar Flow & Distance T/S \\
& {$[\mathrm{nm} / \mathrm{s}]$} & {$[\mathrm{nm} / \mathrm{s}]$} & {$[\mathrm{W}]$} & {$[\mathrm{mbar}]$} & {$[\mathrm{sccm}]$} & {$[\mathrm{cm}]$} \\
\hline $\mathrm{TiNi}$ & 2.32 & 1.13 & 500 & $2.3 \times 10^{-3}$ & 20 & 7 \\
$\mathrm{TiNi}$ & 3.05 & 1.52 & 500 & $2.3 \times 10^{-3}$ & 20 & 6 \\
$\mathrm{TiNi}$ & 3.97 & 2.12 & 500 & $2.3 \times 10^{-3}$ & 20 & 5 \\
$\mathrm{TiNi}$ & 4.44 & 2.35 & 500 & $2.3 \times 10^{-3}$ & 20 & 4.5 \\
$\mathrm{Cu}$ & 3.18 & 2.3 & 400 & $4.0 \times 10^{-3}$ & 20 & 7 \\
$\mathrm{Cr}$ & 2.62 & 1.3 & 400 & $4.0 \times 10^{-3}$ & 20 & 7 \\
$\mathrm{Au}$ & 0.93 & - & 100 & $4.0 \times 10^{-3}$ & 20 & 7 \\
$\mathrm{Pt}$ & 1.1 & - & 200 & $4.0 \times 10^{-3}$ & 20 & 7 \\
$\mathrm{Mo}$ & 1.4 & - & 500 & $4.0 \times 10^{-3}$ & 20 & 7 \\
\hline
\end{tabular}

One of the aims of this research is the fabrication of thin films with greater thickness. Thus, a compromise between the deposition parameters was assumed in order to 
achieve the highest possible deposition rate, also considering the thickness distribution. Distance between target and substrate 7 and $6 \mathrm{~cm}$ were adjusted for planar deposition DC magnetron sputtering. As described in section 2.4.1, the sputter yield is the number of sputtered atoms divided by the number of incident particles and is a criterion for efficiency of sputtering.

The sputtering of TiNi reveals a characteristic loss of $\mathrm{Ti}$, as described in section 2.4.1. To investigate the influence of the distance between target and substrate on the TiNi thin film stoichiometry, as well as the Ti loss, two Si 4 inch wafers were deposited using DC magnetron, target $T i_{53.6} N i_{46.4}, 500 \mathrm{~W}$ power, $2.3 \times 10^{-3}$ mbar pressure and $20 \mathrm{sccm}$ Ar flow. The distance between target and sample was varied $(6$ and $7 \mathrm{~cm})$. The homogeneity of the stoichiometry was measured by means of EDX within a scanning electron microscope (see 3.1.1) in the $\mathrm{x}$ and $\mathrm{y}$ axes, as shown in figure 4.4 . Error bars were determined by standard deviation of repeated measurements and minimized by the use of a certified TiNi standard.

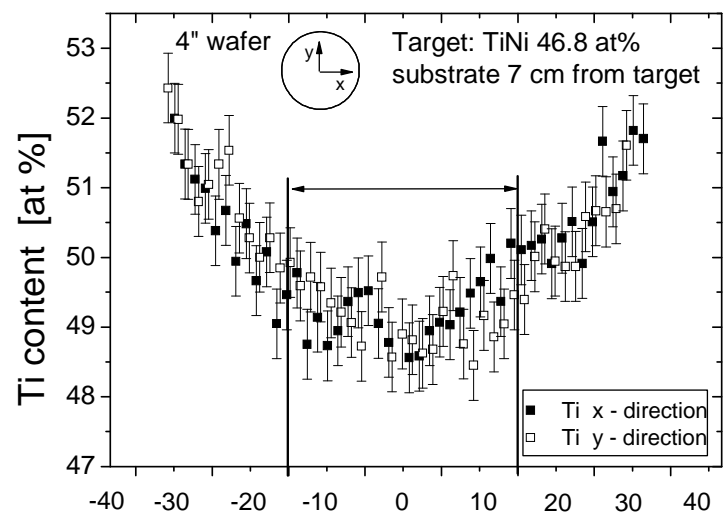

(a) wafer position [mm]

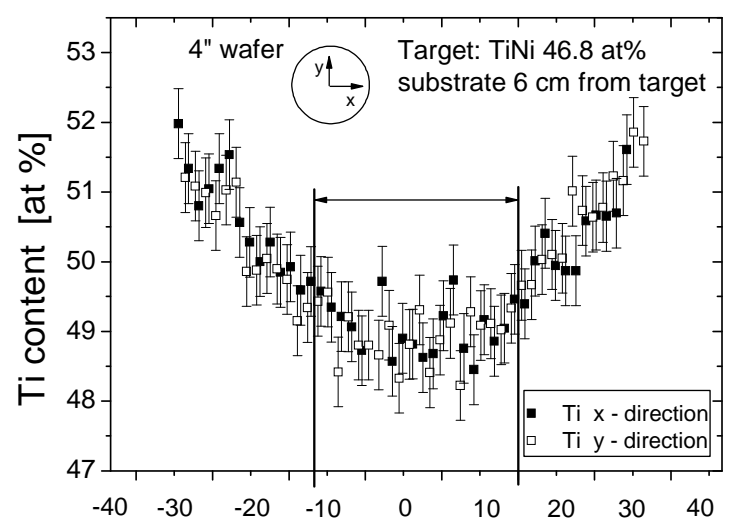

(b) wafer position [mm]

Figure 4.4: (a) EDX measurements along $x$ and $y$ axis of a TiNi thin film deposited on a Si 4 inch wafer at $\mathbf{7} \mathbf{~} \mathbf{m}$ distance to $a T i_{53.6} N i_{46.4}$ at \% target. (b) EDX measurements along $x$ and $y$ axis of a TiNi thin film deposited on a Si 4 inch wafer at $6 \mathbf{c m}$ distance to a $T i_{53.6} N i_{46.4}$ at \% target. Power, vacuum pressure and gas flow were identical for both depositions.

Figure 4.4 (a) shows EDX measurements along $\mathrm{x}$ and $\mathrm{y}$ axes of a TiNi thin film 
deposited on a Si 4 inch wafer at $7 \mathbf{~ c m}$ distance to a $T i_{53.6} N i_{46.4}$ at $\%$ target and figure 4.4 (b) shows EDX measurements along $\mathrm{x}$ and y axes of a TiNi thin film deposited on a Si 4 inch wafer at $6 \mathbf{c m}$ distance to a $T i_{53.6} N i_{46.4}$ at \% target. The deposition parameters were kept the same for both analyzed samples. Within a radius of approximately $45 \mathrm{~mm}$, the sputtered films at $7 \mathrm{~cm}$, revealed an almost constant composition of $T i_{49.1} N i_{50.9}$. This corresponds to an average loss rate of 4.5 at $\% \mathrm{Ti}$ between target and film. The sputtered film at $6 \mathrm{~cm}$ revealed an almost constant composition of $T i_{49.0} N i_{51.0}$, within a radius of approximately $40 \mathrm{~mm}$. This corresponds to an average loss rate of 4.6 at \% Ti between target and film. The radial distribution effect observed is derived from the differential angular distribution of $\mathrm{Ti}$ an $\mathrm{Ni}$, leading to an increase of $\mathrm{Ti}$ content of the film up to $T i_{52} N i_{48}$ at $\%$ at the board of the 4 inch wafer. Thus, the substrate size must be reduced in order to assure the homogeneity stoichiometry along the whole substrate or the distance between target and substrate must be increased.

\subsubsection{Thin film ex situ crystallization and characterization}

As described in section 2.3. Nishida et al. [22] found that the phase $T i_{3} N i_{4}$ appears depending upon aging temperature and time. The formation of such precipitates helps to adjust the transformation temperatures of the martensite transformation [19]. Thus, it is very important to understand the influence of the annealing on the phase transformation and mechanical properties of TiNi thin film shape memory alloy. TiNi thin film ex situ annealing was carried out with RTA (see section 3.2.3) and mechanical properties were characterized with tensile tests, see section 3.1.6. Two series of TiNi thin films were deposited at room temperature on Si stress-strain substrates $(50 \times 3 \mathrm{~mm})$. The deposition parameters used were: $T i_{53.6} N i_{46.4}$ at $\%$ target, $500 \mathrm{~W}$ power (DC), $2.3 \times 10^{-3}$ mbar pressure, $20 \mathrm{sccm}$ Ar flow and $7 \mathrm{~cm}$ distance between target and substrate. The film thickness was approximately $7 \mu \mathrm{m}$. EDX analysis for both samples shows the same stoichiometry $T i_{49.3} N i_{50.7}$ at \%. The first film was annealed for one hour at $500{ }^{\circ} \mathrm{C}$. The second film was annealed in two steps: $1 / 2$ hour at $650{ }^{\circ} \mathrm{C}$ and a further $1 / 2$ hour at $450{ }^{\circ} \mathrm{C}$. Differential scanning analysis was carried out to investigate and determine the 
transformation temperatures. Tensile tests were performed to investigate the influence of the crystallization on the mechanical properties of the films. Figure 4.5 (a) and (b) show DSC analysis and tensile test of the film annealed for one hour at $500{ }^{\circ} \mathrm{C}$, while Figure 4.5 (c) and (d) show DSC analysis and tensile test of the film annealed in two steps.
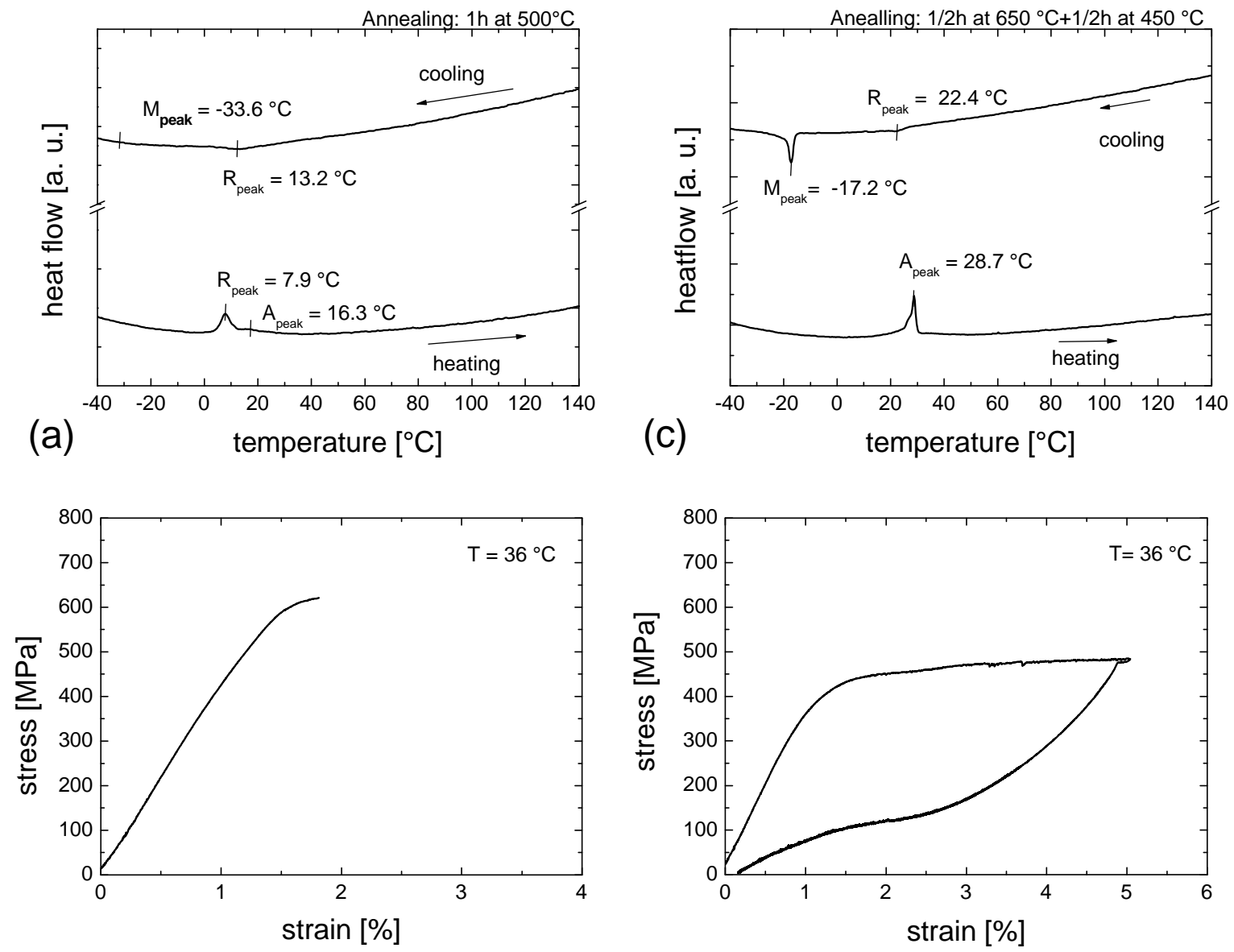

(b)

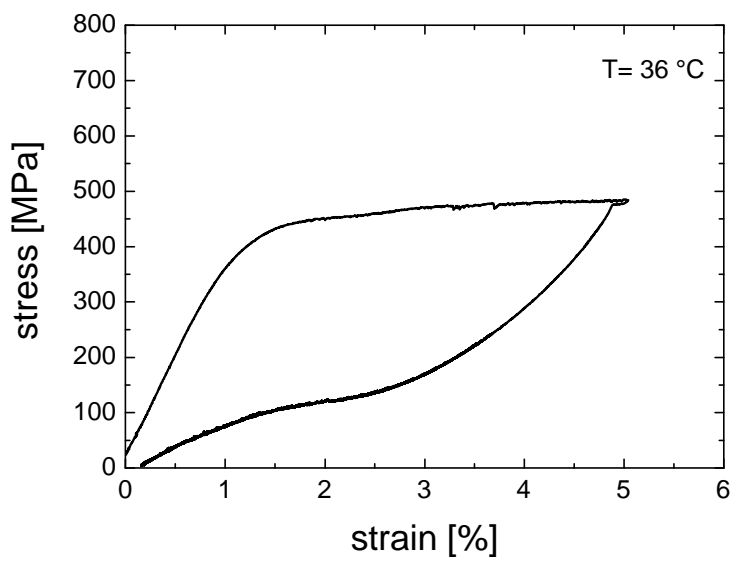

(d)

Figure 4.5: (a) DSC analysis and tensile test (b) of a film annealed for one hour at $500^{\circ} \mathrm{C}$ (c) DSC analysis and tensile test (d) of a film annealed in two steps: $1 / 2$ hour at $650^{\circ} \mathrm{C}$ and further $1 / 2$ hour at $450^{\circ} \mathrm{C}$.

Figure 4.5 (a) shows the DSC result of TiNi films annealed for 1 hour at $500{ }^{\circ} \mathrm{C}$. During heating, a two-stage transformation is observed, from martensite phase to R-phase and from R-phase to austenite, where the R-phase peak is $7.9^{\circ} \mathrm{C}$ and the Austenite peak is 
$16.3^{\circ} \mathrm{C}$. During cooling, a two-stage transformation is also observed, from austenite to $\mathrm{R}$-phase and from R-phase to martensite, where the $\mathrm{R}$-phase peak is $13.2{ }^{\circ} \mathrm{C}$ and the Martensite peak is $-33.6^{\circ} \mathrm{C}$. The tensile test (figure $4.5(\mathrm{~b})$ ) realized on this film shows a linear elastic increase with an E modulus of $32 \mathrm{GPa}$ at a strain of $1.5 \%$. A plastic deformation is observed at $670 \mathrm{MPa}$ and the film is broken.

Figure 4.5 (c) shows the DSC result of TiNi films annealed in two steps. During heating, an one-stage transformation is observed, from martensite to austenite phase, where the Apeak is $28.7^{\circ} \mathrm{C}$. During cooling, a two-stage transformation is observed, from austenite to $\mathrm{R}$-phase and from $\mathrm{R}$-phase to martensite, where the $\mathrm{R}$-phase peak is $22.4{ }^{\circ} \mathrm{C}$ and the Martensite peak is $-17.2^{\circ} \mathrm{C}$. The tensile test, figure 4.5 (d), realized on this film shows a start superelastic plateau of approximately $450 \mathrm{MPa}$ and a closed loop hysteresis with a maximum of $5 \%$ strain. The shift of the austenite peaks from 16.3 to $28.7{ }^{\circ} \mathrm{C}$ is caused by the formation of $T i_{3} N i_{4}$ precipitates, as described in section 2.3, and the removal of intrinsic stresses within the material. Zhang et al. [73] clarified this phenomenon. They found that alternating the heat treatment temperature causes a change in the $\mathrm{B} 2$ matrix composition. Thus, the precipitation reaction phenomenon is used to fine adjust the TiNi matrix composition giving a precise control of temperature transformation.

As, the aim of this dissertation is production, development and characterization of superelastic TiNi thin film for medical application, the film must be superelastic at body temperature $\left(36-37^{\circ} \mathrm{C}\right)$. Thus, the films were mechanically tested at $36{ }^{\circ} \mathrm{C}$. As described in section 2.1.3, superelasticity is the ability of the shape memory alloys to completely recover from an apparently martensitic plastic deformation just by unloading the applied stress at a temperature above $A_{f}$. In the case of the film annealed for one hour at $500{ }^{\circ} \mathrm{C}, 36{ }^{\circ} \mathrm{C}$ is far away from the austenite finish. Thus, the film has a very stable martensite phase, which explains the ultimate tensile strength of $670 \mathrm{MPa}$ and a film failure at $2 \%$ strain. $36{ }^{\circ} \mathrm{C}$ is just above the austenite finish for the film annealed in two step, thus the martensite phase is not stable enough and the stress of $450 \mathrm{MPa}$ is sufficient to induce the martensite transformation achieving a $5 \%$ strain and a close 
loop hysteresis.

To demonstrate the superelastic effect within freestanding films, tensile testing was carried out at different temperatures [74, 75] on TiNi thin films with $10 \mu m$ thickness . Freestanding NiTi films showed a typical martensitic behaviour at $-10{ }^{\circ} \mathrm{C}$ (figure 4.6 (a)) A strain of $5 \%$ was achieved after application of $220 \mathrm{MPa}$ stress which led to a permanent plastic deformation of $3.5 \%$ strain. Upon heating, the plastic deformation recovers and superelastic behaviour appears. Figure 4.6 (b) and (c) show typical superelastic curves repeatedly exceeding an elastic strain of $6.5 \%$ at body temperature, as well as at $57^{\circ} \mathrm{C}$ whereas no stress induced martensitic transformation is achieved when the temperature exceeds $A_{f}$. The stress-strain diagram of the pure austenitic state at $120{ }^{\circ} \mathrm{C}$ is presented in figure 4.6 (d), which shows as a typical result an ultimate tensile strength of 960 $\mathrm{MPa}$. The same experiment carried out below $A_{f}$ is shown in figure 4.6 (e) indicating superelastic strain and an ultimate tensile strength of $1180 \mathrm{MPa}$ at a maximum strain of $11.5 \%$. Both values are among the highest numbers ever published for binary TiNi thin films obtained by magnetron sputtering. Moreover, first fatigue tests were carried out. Tensile testing experiments up to a maximum number of six cycles were performed at $37^{\circ} \mathrm{C}$ (figure 4.6 (f)). After the first cycle a slight decrease of the stress at the beginning of the superelastic plateau was obtained, which then remained constant within the next five cycles of testing.
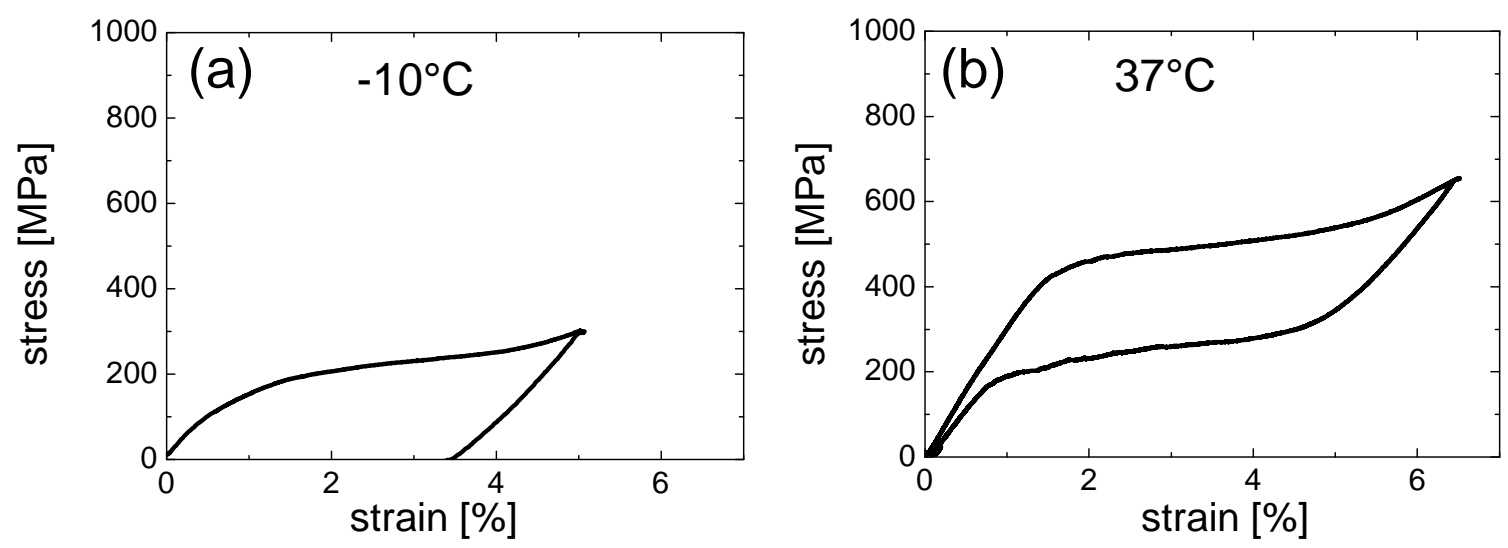

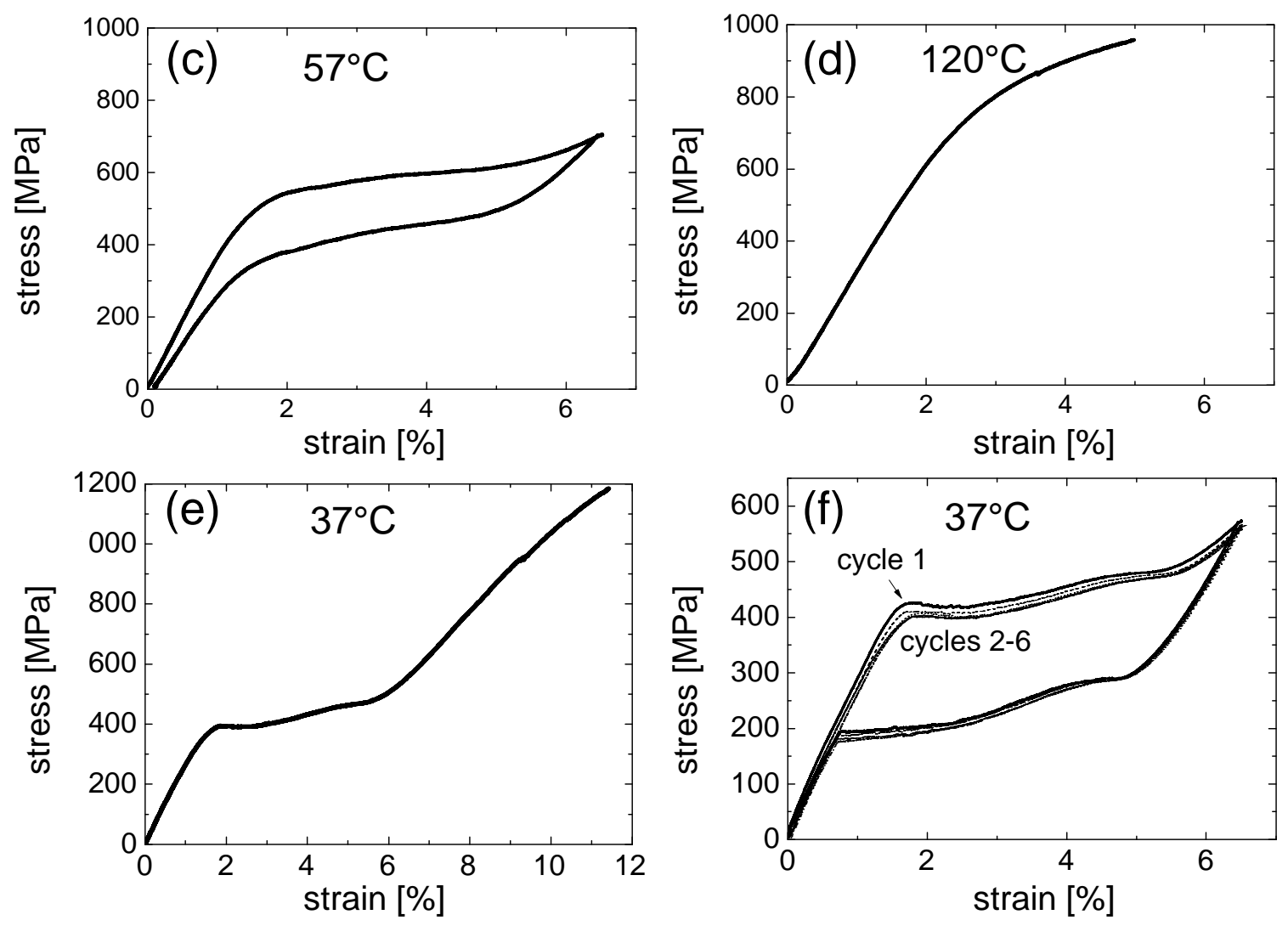

Figure 4.6: Temperature influence on TiNi film superelastic effect [74, 75].

Part of the aim of this disseration is the patterning of cylindrical TiNi thin films by photo lithography and wet etching. The patterned film must contain the same mechanical properties as the one showed by the films before structuring. Thus, the effect of edge quality and the influence of the etchant, e.g. hydrogen embrittlement, on the film properties was analysed. Figure 4.7 shows the tensile tests realized in four TiNi stress-strain films which were deposited and patterned with different geometries. The thickness of the stress-strain TiNi film samples was $11 \mu \mathrm{m}$ and its dimension was $30 \mathrm{~mm}$ length and $4 \mathrm{~mm}$ width for all four samples.

Figure 4.7 shows tensile tests realized on $11 \mu \mathrm{m}$ thickness TiNi thin films structured by lithography and wet etching, with different feature sizes: (a) from 300 to $500 \mu \mathrm{m}$, (b) from 100 to $250 \mu \mathrm{m}$, (c) from 100 to $200 \mu \mathrm{m}$, and (d) $100 \mu \mathrm{m}$. In this analysis, the geometry in the stress calculation was not considered, because the aim of the analysis 

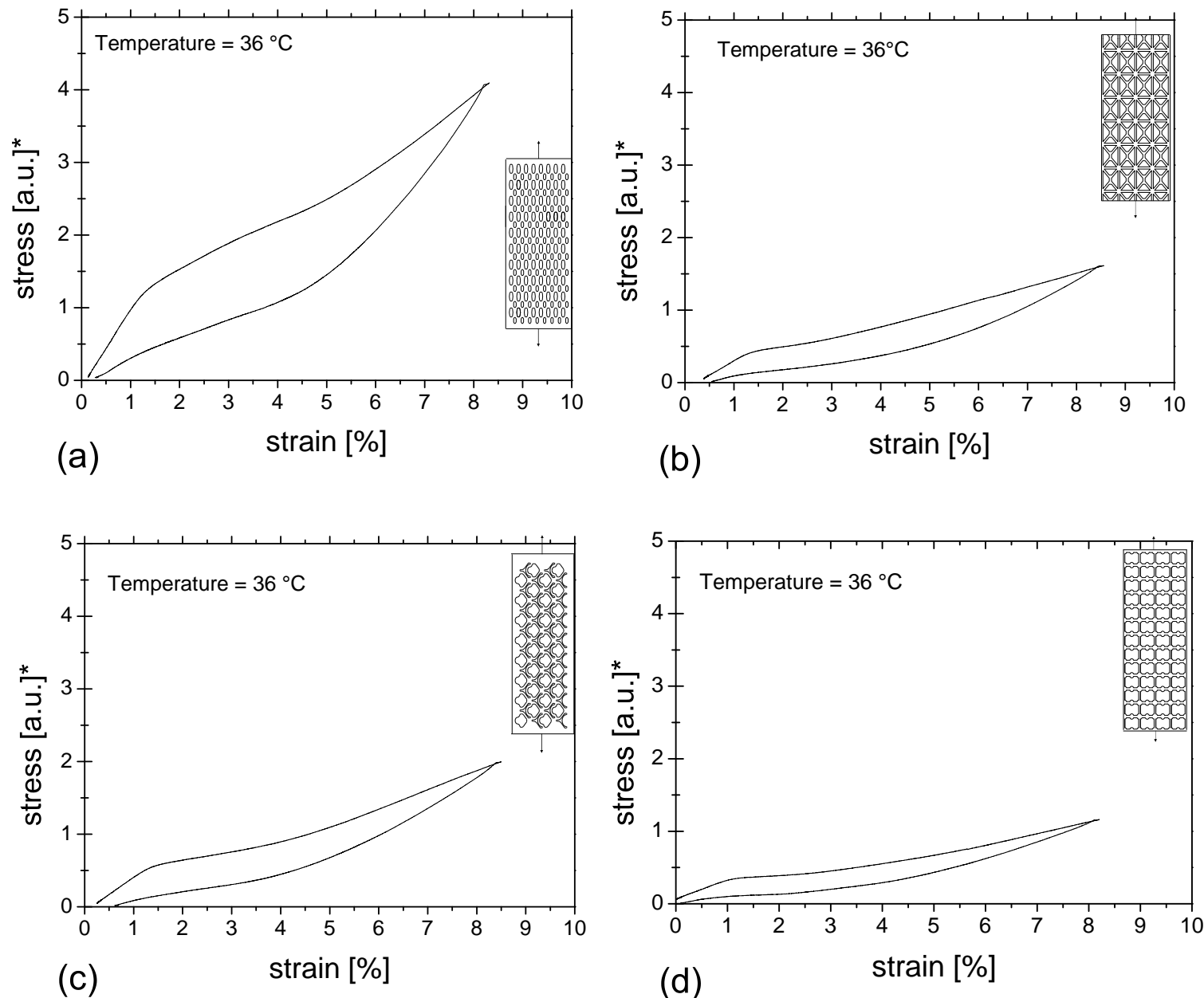

Figure 4.7: Tensile tests realized on $11 \mu \mathrm{m}$ thickness TiNi thin films structured by lithography and wet etching, with different feature sizes: (a) from 300 to $500 \mu \mathrm{m}$, (b) from 100 to $250 \mu \mathrm{m}$, (c) from 100 to $200 \mu \mathrm{m}$, and (d) $100 \mu \mathrm{m}$.

was the characterization of the film properties after structuring and not the influence of the geometry. A closed loop hysteresis is shown in all four structured samples, with a remarkable $9 \%$ strain and a superelastic plateau. No sample contamination or influence of the wet etching process was observed. Thus, mechanical properties achieved after TiNi films structuring are comparable with properties of the film without structuring, as shown in figure 4.5 . 


\subsection{Fabrication and characterization of cylindrical thin films}

The success of stents and other medical implants, which are conventionally fabricated of thin-walled, laser structured tubes based on superelastic TiNi and the limitations regarding the miniaturization of these medical devices, has motivated the application of thin film technology as an attractive alternative. It was developped during this dissertation a way of producing TiNi thin films by magnetron sputtering. This method and characterization of the films will be described in the following section.

\subsubsection{Sputtering device characterization}

Compared with planar deposition, described in the last section, the cylindrical deposition presents many challenges, e.g. film growth on non-planar surfaces, film composition, thickness distribution and mechanical properties. All these issues were addressed, in the course of this dissertation.

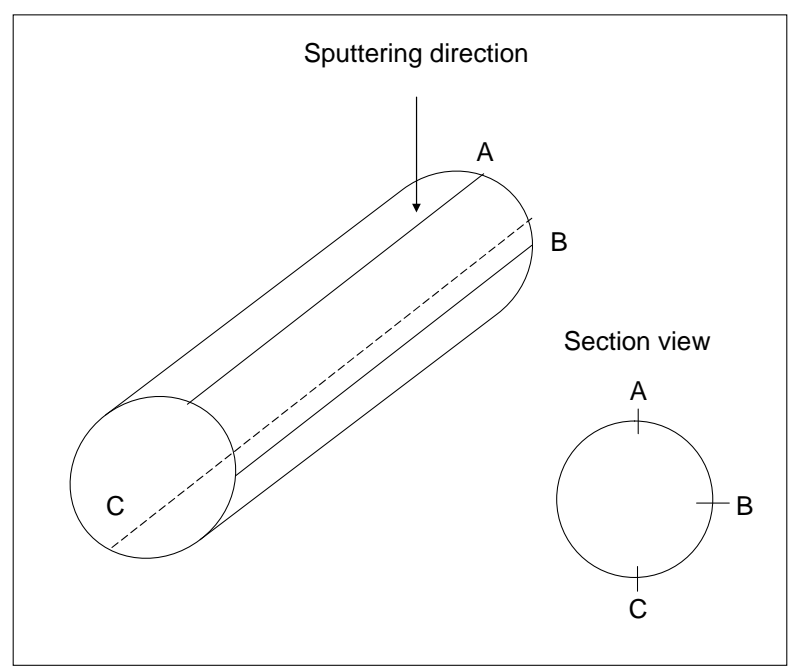

Figure 4.8: Analyzed sample regions. (A) top (B) lateral (C) bottom region.

To understand the film growth on a cylindrical substrate, TiNi thin film was sputtered on a non-rotating cylindrical glass substrate that was firstly covered with sacrificial 
layers. Deposition parameters used were: $T i_{53.2} N i_{46.8}$ at $\%$ target, $500 \mathrm{~W}$ power (DC), $2.3 \times 10^{-3}$ mbar pressure, $20 \mathrm{sccm}$ Ar flow, and distance between target and substrate $=6 \mathrm{~cm}$. The thin film surface microstructure and stoichiometry were analyzed and measured for each side of the sample, as shown in figure 4.8, by means of SEM and EDX. Figure 4.9 shows the stoichiometry and the surface microstructure of a TiNi film sputtered on a non-rotating glass cylinder - (A) top region, (B) lateral region, and (C) bottom region.
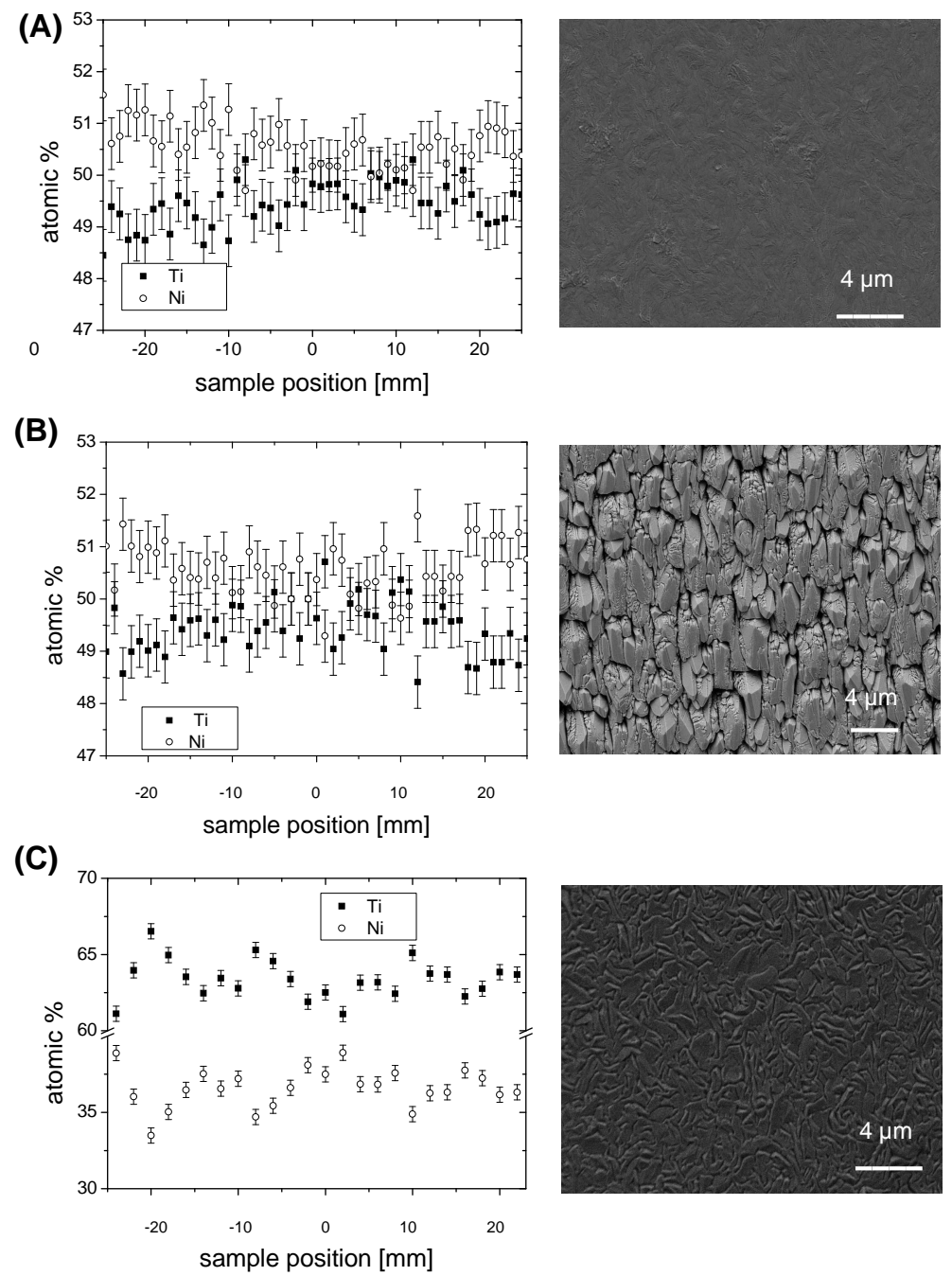

Figure 4.9: Stoichiometry and microstructure of a TiNi film sputtered on a non-rotating glass cylinder - (A) top region, (B) lateral region, and (C) bottom region. 
The top region (figure 4.9) (A) showed a dense microstructure and a stoichiometry average of $50.6 \pm 0.5$ at $\% \mathrm{Ni}$, while the lateral region (B) (fig. 4.9) of the sample showed a columnar microstructure and a stoichiometry average of $50.8 \pm 0.5$ at $\% \mathrm{Ni}$ along the side. The bottom region (C) (fig. 4.9) showed a dense microstructure and a stoichiometry average of $36.3 \pm 0.5$ at $\% \mathrm{Ni}$.

The microstructure of the thin film showed a non-homogeneous growth along the different sides of the sample, which can be explained by the different deposition angles, as described in section 2.4.1. To prove this assumption a dynamic process was developed based on a specific device which allows an in situ rotation of the substrate during the sputtering process.
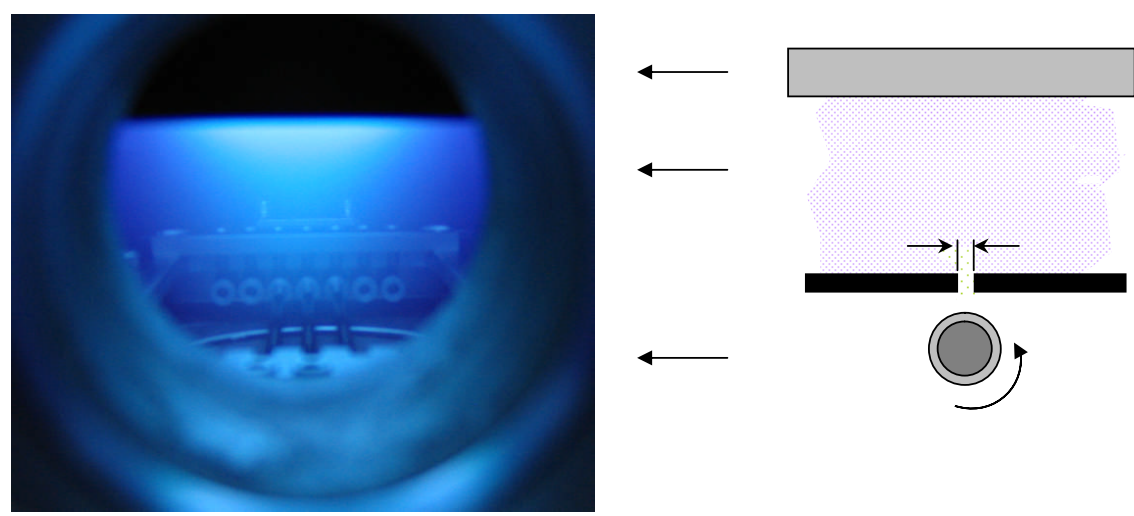

$\longrightarrow$ TiNi target
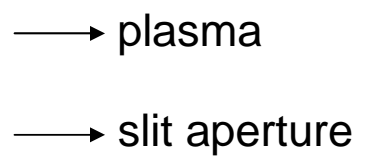

$\longrightarrow$ sample

Figure 4.10: Rotational device installed on the Alcatel 450 sputtering machine.

The rotational device was developped to rotate 7 substrates at the same time with different diameters, which can be between 1 to $5 \mathrm{~mm}$. The rotation can be set up, from 10 to $150 \mathrm{rpm}$, using a vacuum proof electric motor placed inside of the vacuum chamber. Figure 4.10 shows the rotational device installed on the Alcatel 450 sputtering machine during a deposition process and figure 4.11 shows its engineering sketch, where the numbers from 1 to 7 correspond to the sample position.

To confirm the necessity of a dynamic process a cylindrical glass substrate, covered with sacrificial layers beforehand, was mounted on the rotational device and deposited with TiNi. A Focus Ion Beam (FIB) cross section was realized to understand the TiNi 


\section{top view}

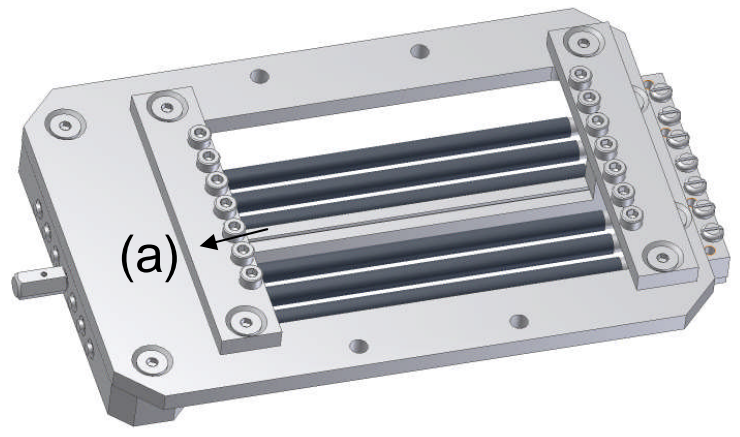

\section{bottom view}

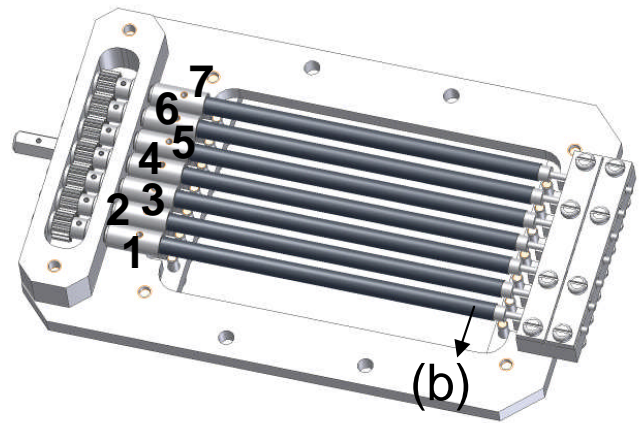

Figure 4.11: Rotational device engineering sketch: (a) slip aperture (b) seven possible substrate position.

thin film microstructure and the stoichiometry homogeneity distribution was measured by means of EDX along the sample, as shown in figure 4.12. The sample was placed in position 3 of the rotational device and deposited using a $T i_{53.2} N i_{46.8}$ at $\%$ target, $500 \mathrm{~W}$ (DC), vacuum pressure $=2.3 \times 10^{-3} \mathrm{mbar}$, Ar flow $=20 \mathrm{sccm}$, distance between target and substrate $=6 \mathrm{~cm}$ and substrate rotational speed $=4 \mathrm{rpm}$.
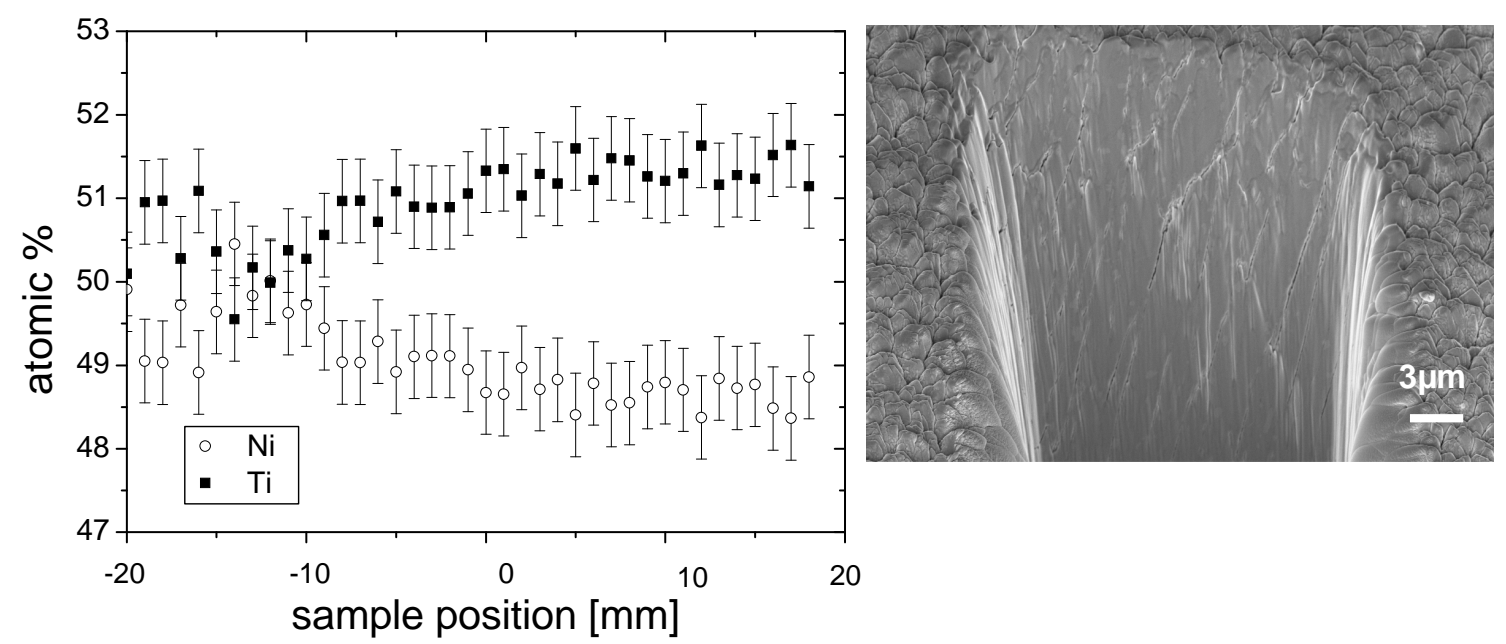

Figure 4.12: Stoichiometry homogeneity distribution and microstructure of an amorphous TiNi film deposited on a cylindrical glass substrate using a rotational device. Cross section realized by Focus Ion Beam (FIB) 
The microstructure of the thin film showed a non-homogeneous growth, which is a result of the sum of the four regions of the previous static deposition (figure 4.8), and a non-homogeneous composition distribution.

To minimize the influence of the deposition angle a shutter was introduced between the magnetron cathode and the cylindrical substrate which was placed as close as possible to the substrate, as shown in figure 4.11. The introduction of this shutter concept allows a homogeneous particle flow normal to the rotating surface. The sample was placed in position 3 of the rotational device and it was deposited using the same parameters as for the previous deposition.

Figure 4.13 shows the stoichiometry homogeneity distribution and microstructure of an amorphous TiNi film deposited on a cylindrical glass substrate using a rotational device and shutter introduced between the magnetron cathode and the cylindrical substrate. The film showed very homogeneous growth and composition distribution due to the introduction of the shutter, which controls the deposition angle allowing homogeneous deposition normal to the rotating surface.
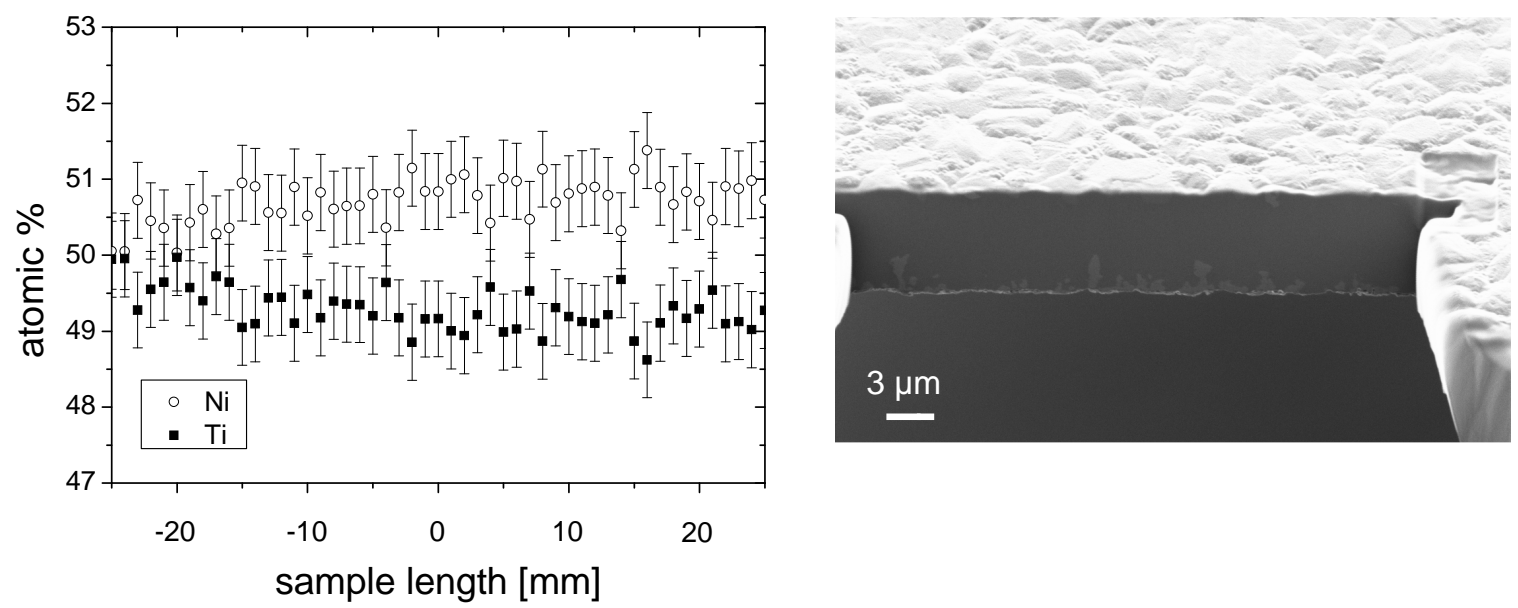

Figure 4.13: Stoichiometry homogeneity distribution and microstructure of an amorphous TiNi deposited on a cylindrical glass substrate using a rotational device and a $0.8 \mathrm{~mm}$ shutter introduced between the magnetron cathode and the cylindrical substrate. Cross section realized by Focus Ion Beam (FIB). 
In order to understand the influence of the shutter width on the deposition rate and stoichiometry three cylindrical glass substrates, $\oslash 5 \mathrm{~mm}$ and length of $100 \mathrm{~mm}$, were assembled into the rotational device in the positions 3,4 and 5 and deposited with TiNi for 1 hour. At each position a shutter with different width was assembled (1.5, 1.0 and $0.8 \mathrm{~mm}$ ). Subsequently, one cylindrical glass substrate, $\oslash 5 \mathrm{~mm}$ and length of 100 $\mathrm{mm}$, was assembled into the rotational device in position 3 and covered with TiNi for 1 hour without shutter. For both experiments the deposition parameters were identical as for the previous experiment. After deposition the films were scratched along the sample length each $10 \mathrm{~mm}$ and the steps between glass and TiNi film were measured by profilometer, see section 3.1 .5 .
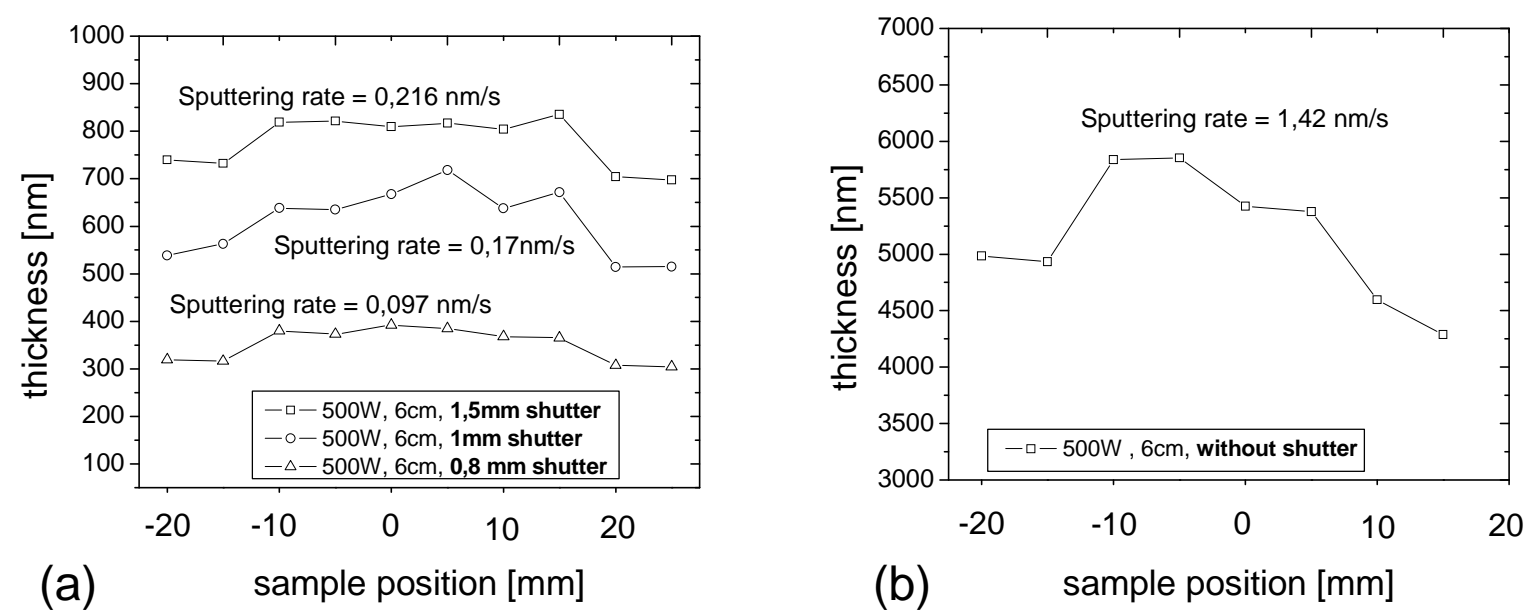

Figure 4.14: (a) Influence of the shutter width on the deposition rate and the deposition distribution along the sample. (b) Deposition rate and the deposition distribution along the sample sputtered without shutter.

Figure 4.14 (a) shows the influence of the shutter width on the deposition rate and the deposition distribution along the sample, while figure 4.14 (b) shows the deposition rate and the deposition distribution along the sample sputtered without a shutter. An average sputtering rate of $0.2 \mathrm{~nm} / \mathrm{s}$ was achieved by using a $1.5 \mathrm{~mm}$ shutter width. Decreasing the shutter width to $0.8 \mathrm{~mm}$ reduces the sputtering rate to $0.1 \mathrm{~nm} / \mathrm{s}$. The homogeneity thickness distribution along the sample sputtered with a shutter is much better than the film sputtered without a shutter. 
The introduction of a shutter decreases the deposition rate by a factor of 15 , in the case of $0.8 \mathrm{~mm}$ shutter width, due to the reduction of the deposition area. However, the film thickness homogeneity (figure 4.14) and the composition distribution (figure 4.13) improve along the sample.

The influence of the shutter width on the film microstructure was analyzed by scanning electron microscopy. Figure 4.15 (a) shows TiNi deposition on cylindrical glass substrates without shutter, (b) TiNi deposition on cylindrical glass substrates with $1.5 \mathrm{~mm}$ shutter, (c) TiNi deposition on cylindrical glass substrates with $0.8 \mathrm{~mm}$ shutter.
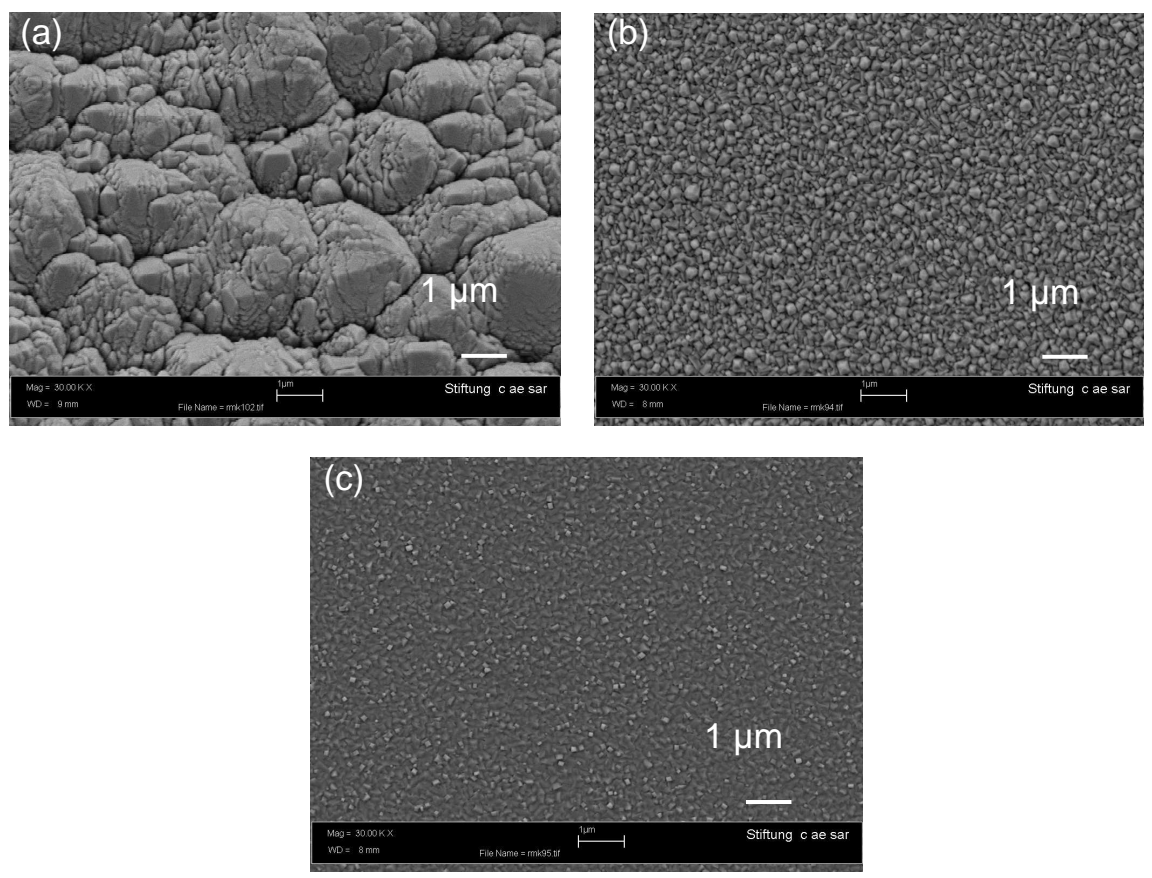

Figure 4.15: (a) TiNi deposition on cylindrical glass substrates without shutter, (b) TiNi deposition on cylindrical glass substrates with $1.5 \mathrm{~mm}$ shutter, (c) TiNi deposition on cylindrical glass substrates with 0.8 mm shutter. Parameters: $\mathrm{Ti}_{53.2} \mathrm{Ni}_{46.8}$ at \% target, $500 \mathrm{~W}(\mathrm{DC})$, vacuum pressure $=2.3 \times 10^{-3}$ mbar, Ar flow $=20 \mathrm{sccm}$, distance between target and substrate $=6 \mathrm{~cm}$, and substrate speed $=4 \mathrm{rpm}$.

The surface of the film sputtered without shutter is very rough compared with the films deposited with 1.5 and $0.8 \mathrm{~mm}$ shutter width. This phenomenon, as described before is 
related to the deposition angle. The influence of the substrate rotational speed on the film microstructure, deposition rate and stoichiometry were also analyzed. Comparing the results obtained, no significant difference was observed on sputtering rate, thickness distribution, film composition and morphology. However, the mechanical wear from the high speed substrate is much higher than the low speed.

The strong dependence of TiNi thin film mechanical properties on the substrate surface quality is already known from the literature [76]. The necessity of miniaturization forces the film surface quality to achieve a good smoothness level. Thus, the influence of substrate roughness and its sacrificial layers on the TiNi thin film growth were analyzed. A cylindrical borosilicate glass was chosen as substrate because of its high degree of smoothness and low concentration of defects.

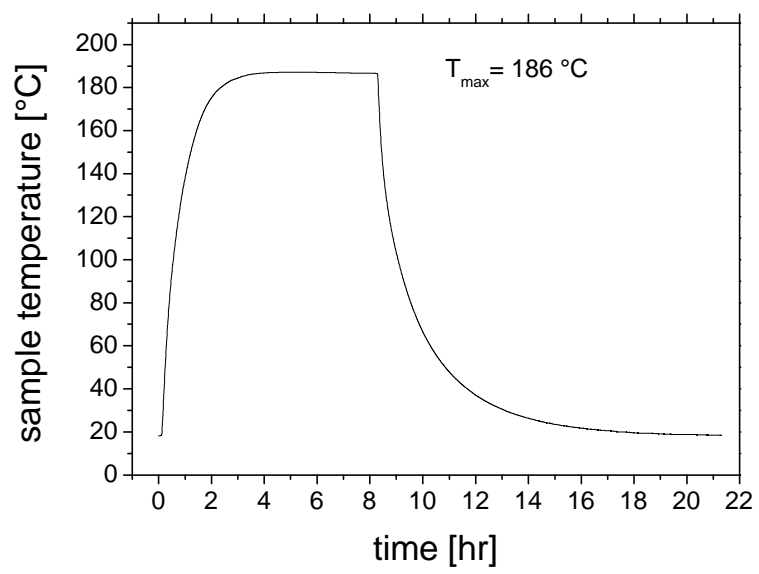

Figure 4.16: Surface temperature path of a cylindrical borosilicate glass substrate deposited with TiNi. Sputtering parameters: $\mathrm{Ti}_{53.2} \mathrm{Ni} i_{46.8}$ at \% target, $500 \mathrm{~W}(D C)$, vacuum pressure $=2.3 \times 10^{-3}$ mbar, Ar flow $=20 \mathrm{sccm}$, distance between target and substrate $=6 \mathrm{~cm}$, substrate speed $=4 \mathrm{rpm}$, and shutter width $0.8 \mathrm{~mm}$.

The fabrication of thin films, specially shape memory alloys, requires the remotion of the deposited thin film from the substrate, for several reasons i.e. characterization, patterning, annealing, application, etc. As a well known technology, sacrificial layer technique is applied and consists of the deposition of a layer or multilayer between the 
substrate and the functional layer. The sacrificial layers are then selectively wet etched in order to get freestanding films. In this dissertation, sacrificial layer technology was applied because glass (substrate) and TiNi are not selective wet etched. Although amorphous TiNi thin films are deposited at room temperature, substrates temperature can be as high as $190{ }^{\circ} \mathrm{C}$ depending on the sputtering conditions due to the deposition energy. Figure 4.16 shows a surface temperature path of a substrate during 10 hours sputtering. A K-type thermoelement was placed inside the deposited cylindrical borosilicate glass substrate. This temperature was taken into consideration for the optimization of the sacrificial layers, once sacrificial layers such as Copper, can recrystalize, increasing its grain size and changing the roughness of the surface.
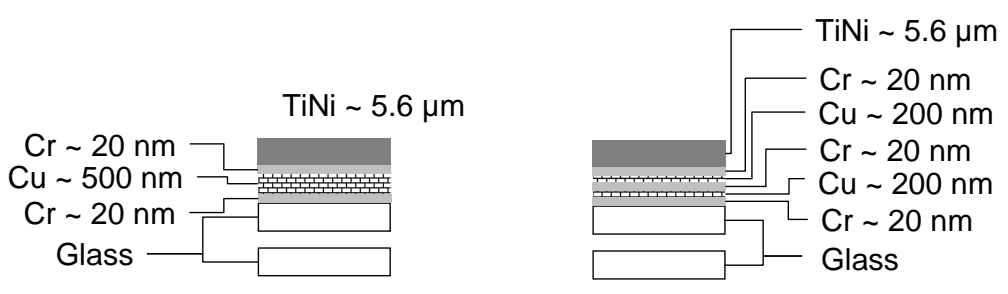

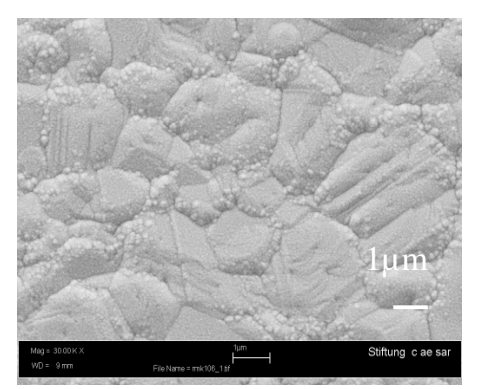

(a)

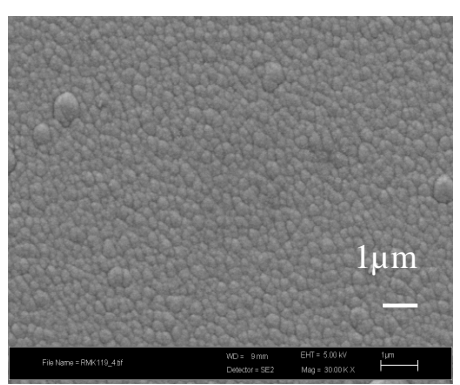

(b)

Figure 4.17: Sacrificial layer smoothness optimization (a) SEM image of a glass substrate sputtered with $\mathrm{Cr} 20 \mathrm{~nm}, \mathrm{Cu} 500 \mathrm{~nm}, \mathrm{Cu} 20 \mathrm{~nm}$ and $5.6 \mu \mathrm{m}$ TiNi and (b) SEM image of a glass substrate sputtered with a multilayer $\mathrm{Cr} / \mathrm{Cu} / \mathrm{Cr} / \mathrm{Cu} / \mathrm{Cr}, \mathrm{Cr} 20 \mathrm{~nm}, \mathrm{Cu} 200 \mathrm{~nm}$ and $5.6 \mu \mathrm{m}$ TiNi.

Two common sacrificial layers were chosen for this investigation: Copper (thermal expansion $=16.5 \times 10^{-6} /{ }^{\circ} \mathrm{C}$ ) and Chromium (thermal expansion $=4.9 \times 10^{-6} /{ }^{\circ} \mathrm{C}$ ). It was observed during previous practical studies, that the sacrificial layers should have at least $500 \mathrm{~nm}$ total thickness, in order to get free standing cylindrical films. Figure 
4.17 shows TiNi surface morphology deposited on: (a) multilayer $\mathrm{Cr} / \mathrm{Cu} / \mathrm{Cr}, \mathrm{Cr} 20 \mathrm{~nm}$, $\mathrm{Cu} 500 \mathrm{~nm}, \mathrm{Cu} 20 \mathrm{~nm}$ and (b) multilayer $\mathrm{Cr} / \mathrm{Cu} / \mathrm{Cr} / \mathrm{Cu} / \mathrm{Cr}, \mathrm{Cr} 20 \mathrm{~nm}$ and $\mathrm{Cu} 200 \mathrm{~nm}$.

$\mathrm{Cr}$ layers were deposited to increase the adhesion and to prevent the diffusion of $\mathrm{Cu}$ into NiTi thin film [77]. The $\mathrm{Cu}$ sacrificial layer roughness is directly related to its thickness [78] and substrate temperature, which in the case of TiNi is approximately $190{ }^{\circ} \mathrm{C}$. Thus, to decrease the $\mathrm{Cu}$ thickness, reducing the surface roughness, multilayer of $\mathrm{Cu}$ and $\mathrm{Cr}$ were used. The reduction on the TiNi surface roughness is clearly observed in figure 4.17 (a) and (b).

\subsubsection{Cylindrical thin film ex situ crystallization}

As shown in section 4.1.2, phase transformation and mechanical properties of TiNi thin film shape memory alloy depend on the crystallization time and temperature. It is known that long-term post-annealing and the aging process could cause dramatic changes to film microstructure, mechanical properties and shape memory effects. The crystallization mechanism in a planar TiNi thin film released from its substrate is well known and depends on the film thickness, thermal history and residual stress [79, 80, 81, 82].

Development of hybrid medicine devices combines conventional production methods (see section 2.5) with thin film technology [83]. As described in section 2.3, to crystallize TiNi thin films temperatures up to $650{ }^{\circ} \mathrm{C}$ and annealing times up to 30 minutes are necessary. These high temperatures/times will change the properties of the conventional TiNi device. Thus, it is necessary to reduce the annealing time in case of a hybrid device development. The crystallization mechanisms as a function of film thickness, annealing time and substrate were analyzed in cylindrical deposited TiNi thin film.

Four series of samples were prepared for this study. All four cylindrical amorphous TiNi thin films were deposited on a $100 \mathrm{~mm}$ long, $3.5 \mathrm{~mm}$ diameter and $0.6 \mathrm{~mm}$ wall thickness polished $\mathrm{Cu}$ rod using a $0.8 \mathrm{~mm}$ shutter channel width, as described in the previous section. Polished $\mathrm{Cu}$ substrate was used due to the need to get the cylindrical TiNi thin film, without patterning, free from the substrate. Two substrates were deposited with $3.5 \mu m$ and two with $8 \mu m$ TiNi film, and cut each into 5 pieces, as shown 
in figure 4.18. The deposition parameters were kept alike varying only the deposition time $\left(T i_{53.2} N i_{46.8}\right.$ at $\%$ target, $500 \mathrm{~W}(\mathrm{DC})$, vacuum pressure $=2.3 \times 10^{-3} \mathrm{mbar}$, Ar flow $=20 \mathrm{sccm}$, distance between target and substrate $=6 \mathrm{~cm}$, and substrate speed $=4 \mathrm{rpm})$.

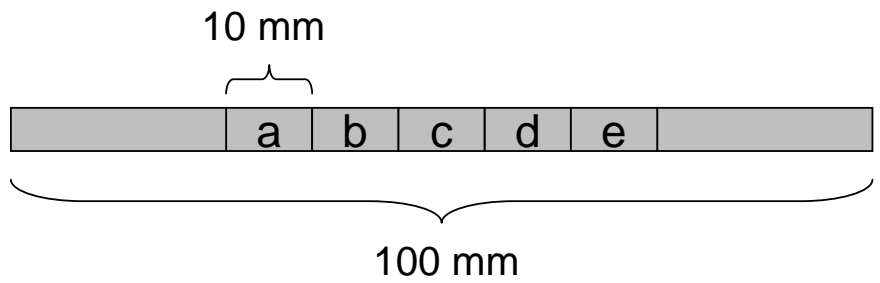

Figure 4.18: Sketch of sample preparation for annealing: $100 \mathrm{~mm}$ long polished Cu substrate deposited with TiNi film.

After cutting, one $\mathrm{Cu}$ rod with $3.5 \mu m$ TiNi film and one with $8 \mu m$ were etched using a 1:1 solution HNO3 and DI water in order to get free standing films. The films were annealed using a rapid thermal annealing (RTA) system. The temperature was the same for all samples, based on the results of the planar film crystallization, see section 4.1.2, only the annealing time was changed. Table 4.2 shows the annealing time for different samples.

Table 4.2: Annealing time for each cut sample. The samples label (a to e)

\begin{tabular}{ccccc} 
corresponds to the labels in figure & 4.18 & & & \\
\hline sample & temperature ${ }^{\circ} \mathbf{C}$ & time & temperature ${ }^{\circ} \mathbf{C}$ & time \\
\hline a & 650 & 30 & 450 & 30 \\
\hline b & 650 & 5 & 450 & 5 \\
\hline $\mathrm{c}$ & 650 & 2.5 & 450 & 2.5 \\
\hline $\mathrm{d}$ & 650 & 1 & 450 & 1 \\
\hline
\end{tabular}

e as deposited - amorphous

Room temperature XRD tests were conducted in four groups of samples $(\mathrm{a}-\mathrm{d})$ : $3.5 \mu \mathrm{m}$ thickness with substrate, $3.5 \mu \mathrm{m}$ without substrate, $8 \mu \mathrm{m}$ with substrate and 
$8 \mu \mathrm{m}$ without substrate. Figure 4.19 shows the influence of the annealing time, thickness and substrate on the crystallization process of cylindrical TiNi thin films, where (a) corresponds to XRD spectra of a cylindrical TiNi films annealed in two steps, $650{ }^{\circ} \mathrm{C}$ for 30 minutes and $450{ }^{\circ} \mathrm{C}$ for further 30 minutes, (b) $650{ }^{\circ} \mathrm{C}$ for 5 minutes and $450{ }^{\circ} \mathrm{C}$ for further 5 minutes, (c) $650{ }^{\circ} \mathrm{C}$ for 2.5 minutes and $450{ }^{\circ} \mathrm{C}$ for further 2.5 minutes and (d) $650{ }^{\circ} \mathrm{C}$ for 1 minute and $450{ }^{\circ} \mathrm{C}$ for a further 1 minute.

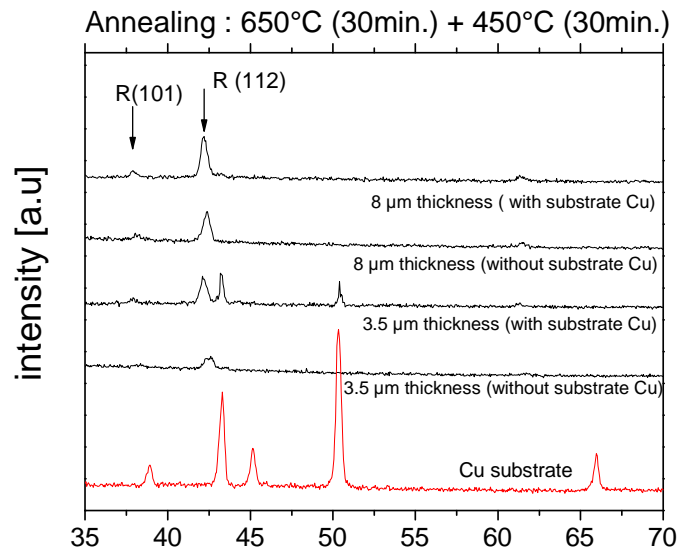

(a)

Annealing : $650^{\circ} \mathrm{C}(2.5 \mathrm{~min})+.450^{\circ} \mathrm{C}(2.5 \mathrm{~min}$.

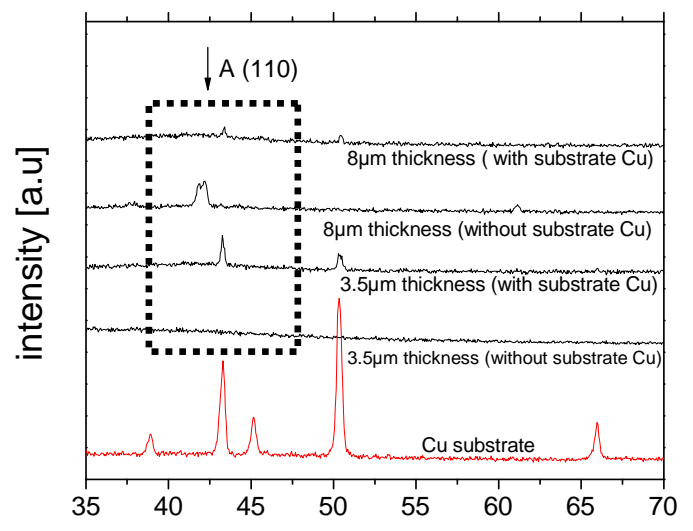

(c)

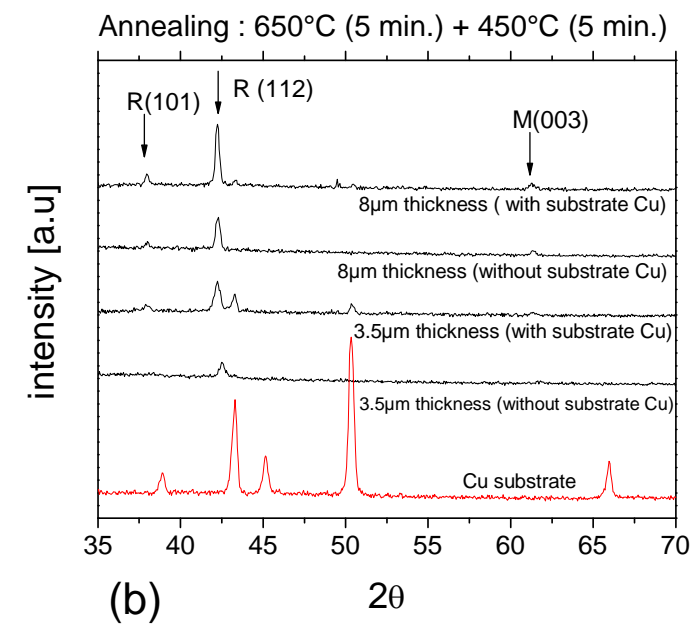

Annealing : $650^{\circ} \mathrm{C}(1 \mathrm{~min})+450^{\circ} \mathrm{C}(1 \mathrm{~min}$.

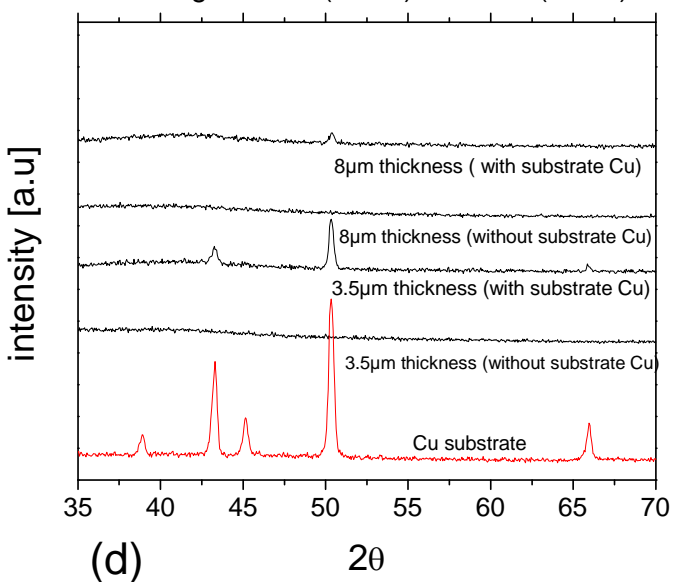

Figure 4.19: Influence of the annealing time, thickness and substrate on the crystallization process of cylindrical TiNi thin films. XRD patterns of four sample groups: $3.5 \mu \mathrm{m}$ with substrate, $3.5 \mu \mathrm{m}$ without substrate, $8 \mu \mathrm{m}$ with substrate and $8 \mu \mathrm{m}$ without substrate. (a) Annealing time 30 minutes, (b) 5 minutes, (c) 2.5 minutes and (d) 1 minute. 
The peaks of the crystalline films can be observed in all sample series after annealing time of 30 minutes and 5 minutes. At 2.5 minutes only the sample with the configuration - $8 \mu m$ film thickness, annealed without substrate - do not show a sharp crystalline peak. This feature indicates that TiNi thin film exhibited partial crystallization. At 1 minute annealing time the films are not crystallized. Figure 4.20 shows a close zoom on the XRD patterns presented previously (figure 4.19(c)), where the main influences observed on the cylindrical TiNi film crystallization process are presented.

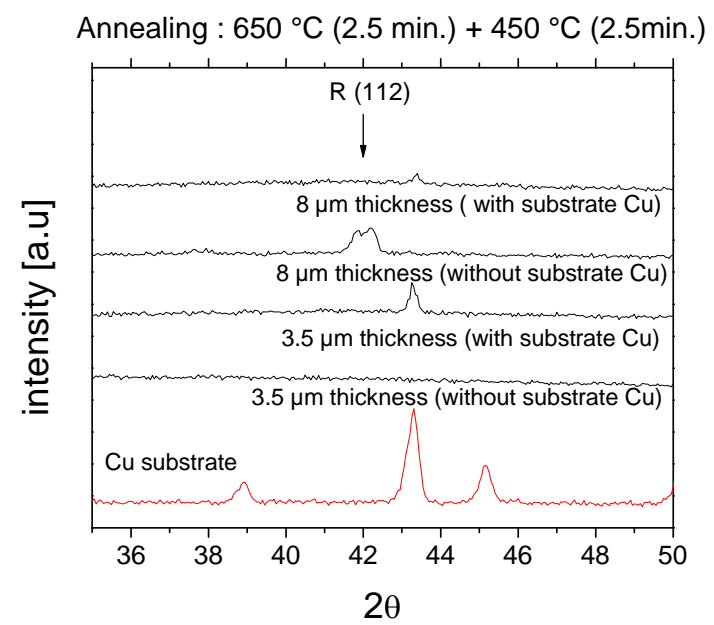

Figure 4.20: Zoom of 4.19 (c) XRD patterns.

TEM (Transmission Electron Microscope) technique was used to understand the microstructure and texture of the $8 \mu \mathrm{m}$ film annealed without substrate for 2.5 minutes. Thus, four samples were prepared and analyzed: (1) 30 minutes annealed without substrate TiNi cylindrical thin film, (2) 30 minutes annealed with substrate TiNi cylindrical thin film, (3) 2.5 minutes annealed without substrate TiNi cylindrical thin film, (4) 2.5 minutes annealed with substrate TiNi cylindrical thin film.

TEM images 4.21 (a) and (b) reveal inhomogeneous microstructures which can occur due to multiple nucleation and growth, some nucleation and growth from surface and some from center of the sample, each one with different kinetic parameters [84] [85] [86]. No difference between the 30 minutes annealed samples with substrate and without substrate was observed. 

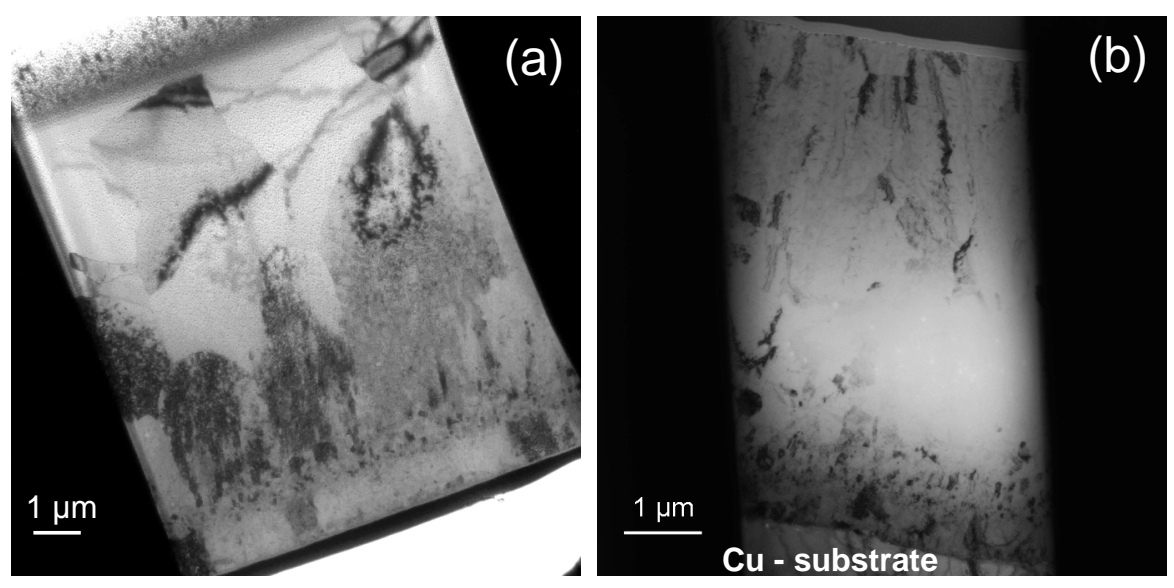

Figure 4.21: TEM cross section of a TiNi cylindrical thin film $8 \mu \mathrm{m}$ thickness(a) 30 minutes annealed without Cu substrate (b) 30 minutes annealed with $\mathrm{Cu}$ substrate.

Figure 4.22 compares the microstructure of two TiNi cylindrical thin film of $8 \mu \mathrm{m}$ thickness: (a) 2.5 minutes annealed without $\mathrm{Cu}$ substrate, (b) 2.5 minutes annealed with $\mathrm{Cu}$ substrate.
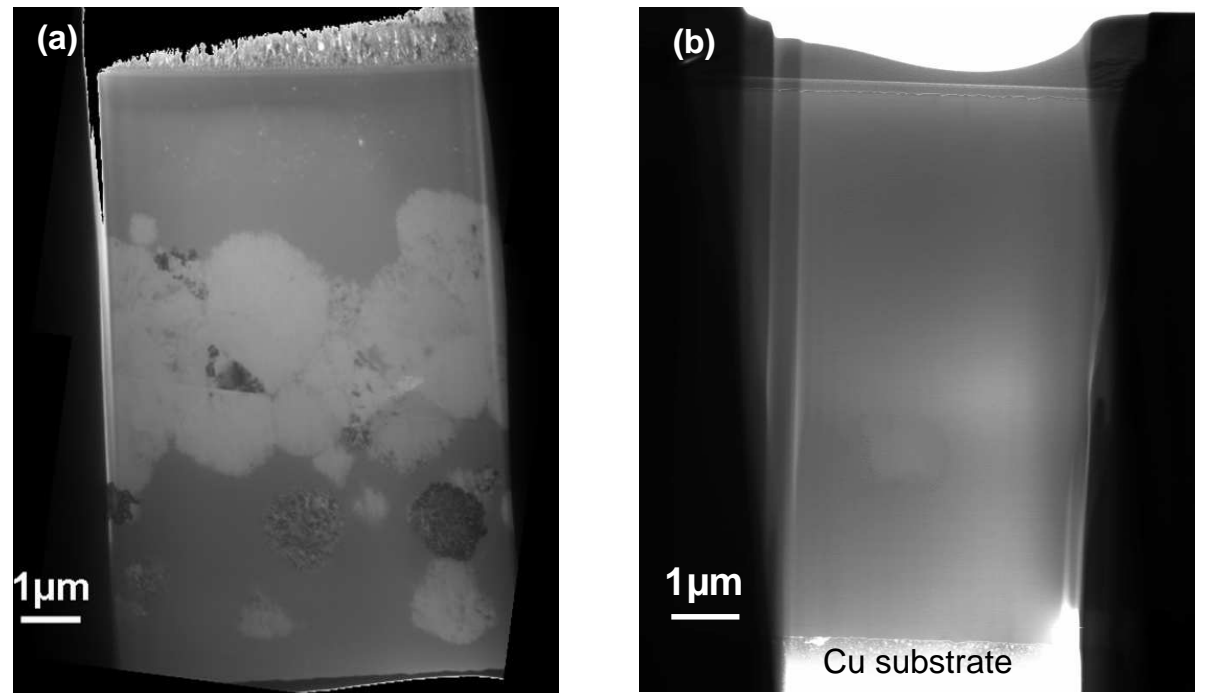

Figure 4.22: TEM cross section of a TiNi cylindrical thin film $8 \mu \mathrm{m}$ thickness (a) 2.5 minutes annealed without Cu substrate (b) 2.5 minutes annealed with Cu substrate.

The sample which was annealed for 2.5 minutes without substrate showed an in- 
complete nucleation, while the one annealed with $\mathrm{Cu}$ substrate showed no crystalline structure, confirming the XRD results. In the first case, crystallization has not reached the film surface and amorphous regions remain between crystalline and film structure. There are two possible reasons for this crystallization: (1) Due to radiation heat provided by the RTA process (see section 3.2.3) the film annealed without substrate tends to absorb more heat than the film annealed with $\mathrm{Cu}$ substrate as described in the specific heat capacity theory [87]. Thus, $2.5 \mathrm{~min}$ seems to be enough time to start the nucleation in the film without substrate. (2) Grummon et al. reported that compressive stresses enhance the crystallization, because the crystallized film reduces its volume and the compressive stress helps the reduction of the crystallized volume [36]. It seems a reasonable explanation for the partial crystallization of the $8 \mu \mathrm{m}$ thickness film annealed for $2.5 \mathrm{~min}$, which should have higher intrinsic stress than the non-crystallized $3.5 \mu \mathrm{m}$ thickness film annealed for $2.5 \mathrm{~min}$ at two steps, $650{ }^{\circ} \mathrm{C}$ and $450{ }^{\circ} \mathrm{C}$. Further TEM analysis in another region of the partial crystallized sample showed a different crystallization evolution. The crystallization in this sample region started from the free surfaces unlike the middle crystallization from the other region. Thus, we can conclude that the crystallization of the sample which was annealed for 2.5 minutes without substrate showed a non-complete heterogeneous nucleation.

One aim of this study was the reduction of the annealling time due to the development of hybrid medicine devices. We can conclude, based on XRD/TEM analysis that 10 minutes is the minimum time required to crystallize cylindrical TiNi thin films using two steps annealing proceedure: 5 minutes at $650{ }^{\circ} \mathrm{C}$ and 5 minutes at $450{ }^{\circ} \mathrm{C}$.

\subsubsection{Mechanical characterization of cylindrical thin films}

Two methods were utilized to investigate TiNi cylindrical thin films mechanical properties: Tensile test and a developed hoop force test used to qualitatively characterize TiNi stents devices.

Once the parameters were optimized, as shown in section 4.2.1, the cylindrical films must be characterized. The tensile test is a well known method of determining me- 
chanical properties of a material. Tensile tests of freestanding films were performed in a universal tensile test device (Messphysik UTM Beta EDC 100N) with the sample being located within a temperature chamber (Shimadzu TCL-N382), as described in section 3.1.6. Thin walled geometries need a very complex tensile test holder. Thus, an alternative method was developed based on lithography and wet etching technology (see sections 2.4.2 and 2.4.3). At first, the sample was deposited using the optimized parameters described in section 4.2.1. After sputtering the sample was structured using cylindrical lithography. This new lithographic approach will be described later. The patterning sketch is shown in figure 4.23. Samples were prepared in radial (r) and axial (a) directions in order to compare the mechanical properties of the film in both directions.

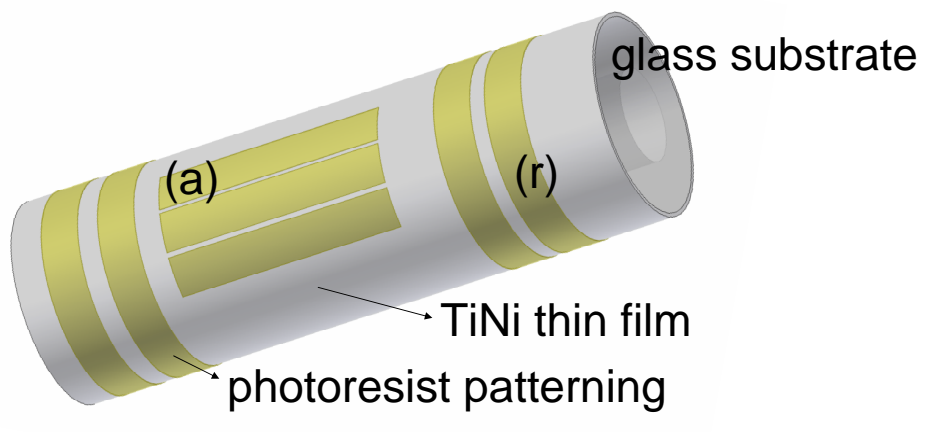

Figure 4.23: Structured photo resist using cylindrical lithography, before TiNi wet etching and crystallization. (r) radial direction sample, (a) axial direction sample.

After lithography, the TiNi thin film was etched with a HF etchant solution for approximately 10 minutes. The sacrificial layers were etched setting the TiNi film free from the substrate. The TiNi films were then crystallized following the same parameters as for the planar TiNi thin films described in section 4.1.2. A pre-load of $0.2 \mathrm{~N}$ was applied in order to assure that the film is pre-stretched and well fixed. A constant strain speed of $0.4 \% / \mathrm{min}$ was applied. The film dimensions were $11 \times 3 \mathrm{~mm}$ and 6.5 $\mu m$ thickness. Figure 4.24 shows the tensile test of a TiNi thin film structured in axial direction. Figure 4.25 shows the tensile test of a TiNi thin film structured in the radial direction. Tensile test parameters were identical for both experiments. 

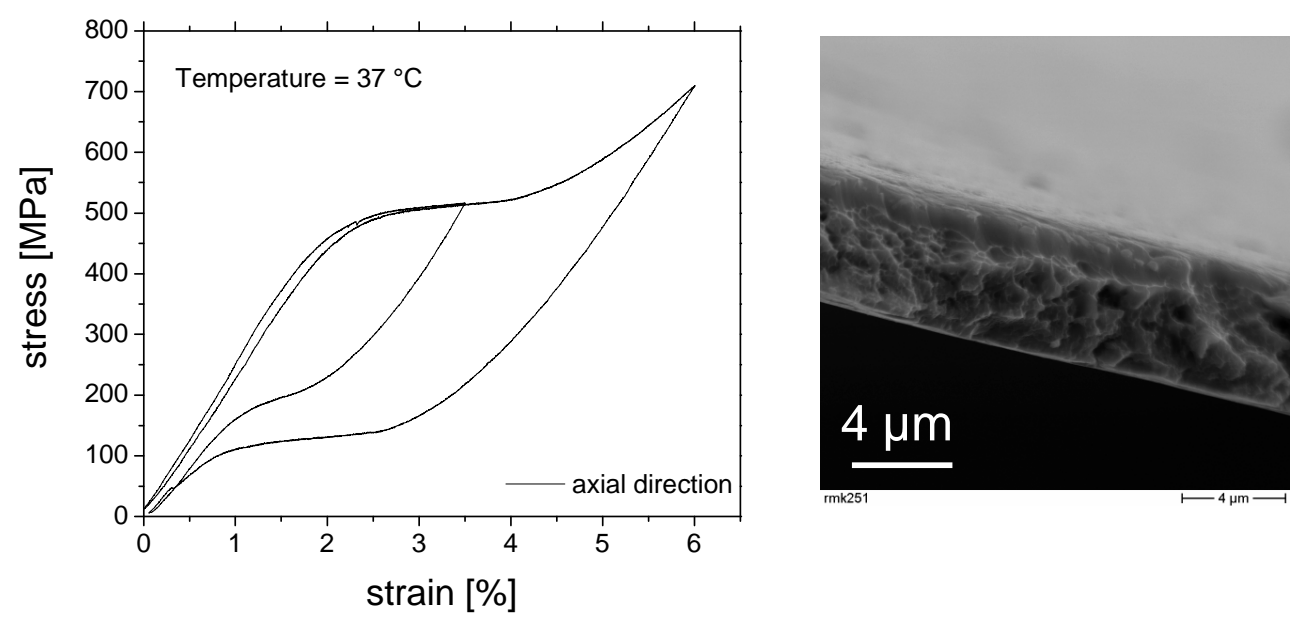

Figure 4.24: Tensile test and microstructure of a TiNi thin film cylindrical sputtered, patterned in axial direction.

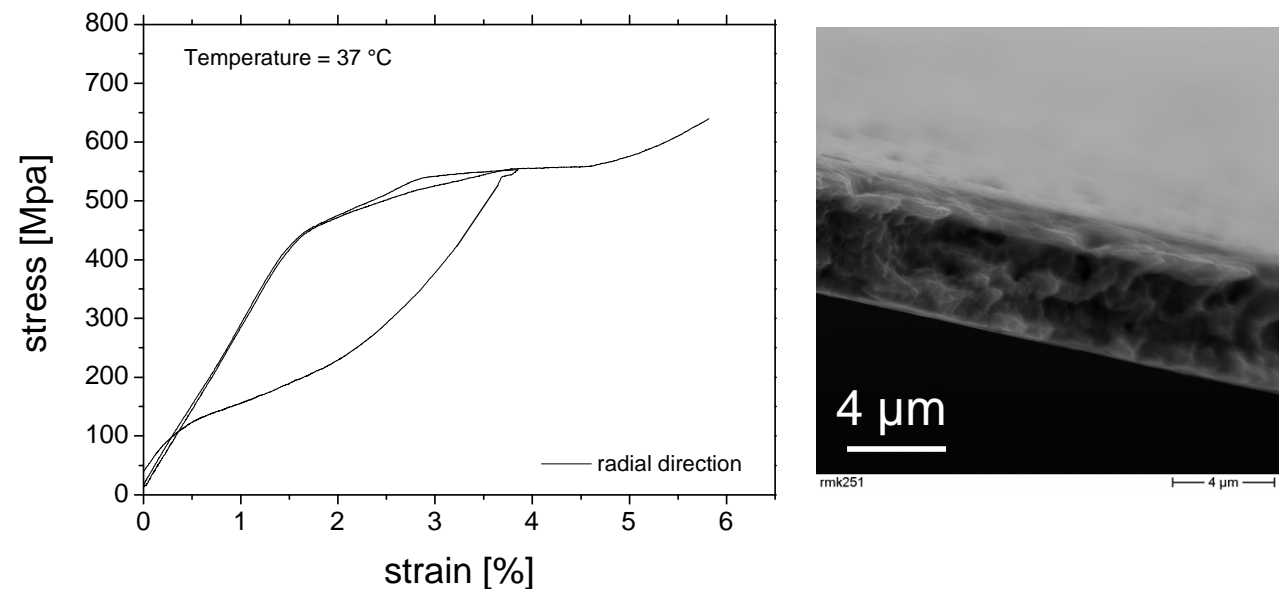

Figure 4.25: Tensile test and microstructure of a TiNi thin film cylindrical sputtered, patterned in radial direction.

The cylindrical TiNi films deposited show in axial and radial direction comparable mechanical properties with TiNi planar films, see section 4.1.2. Superelastic plateau, closed loop hysteresis and a strain up to $6 \%$ were observed. The microstructure of the breaking edges for both samples showed a ductile and dense microstructure.

Hoop force or radial force is a pressure applied in any cylindrical structure, as shown in figure 4.26 [88]. Both the applied pressure and the resulting hoop stress have units of force per unit area, but differ in direction. 


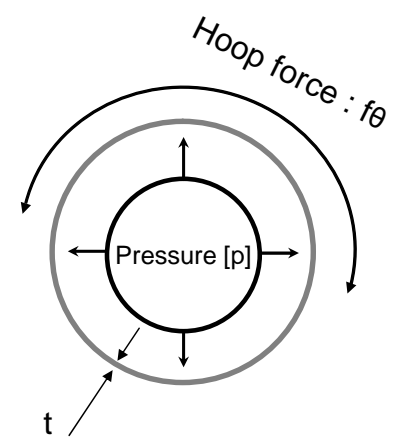

Figure 4.26: Hoop or radial force concept.

In thin-walled cylindrical objects such as stents, the pressure (p) and tensile hoop stress $(\sigma)$ are related by

$$
\sigma=\frac{p \cdot r}{t}
$$

where $r$ is the stent radius and $t$ is the stent thickness. The hoop force $F_{\theta}$ can be described in a vessel wall as:

$$
F_{\theta}=\sigma t L=p r L
$$

where $L$ is the stent length. It is more convenient to define hoop force per unit of length. Thus, we can describe hoop force $\left(f_{\theta}\right)$ as:

$$
f_{\theta}=\frac{F_{\theta}}{L}=\sigma t=p r
$$

Based on the hoop force principle, we developed a method (figure 4.27) to measure stent diameter changes as a function of the applied pressure and temperature. A heater chamber, figure 4.27 (a), was mounted between an optical micrometer (Keyence LS 7030R) with a $0.05 \mu \mathrm{m}$ resolution, figure 4.27 (b). A stent was mounted over a latex tube, 4.27 (e), which was fixed between two steel tubes. A valve was installed at the outlet tube, figure 4.27 (c), and an air pressure calibrator (USF Wallace and Tiernan 
DPR 20C) with a 0.001 bar resolution was installed at the inlet, figure 4.27 (d). The optical micrometer and the pressure calibrator were controlled by self developed software, where the desired pressure, wall thickness and diameter were entered.

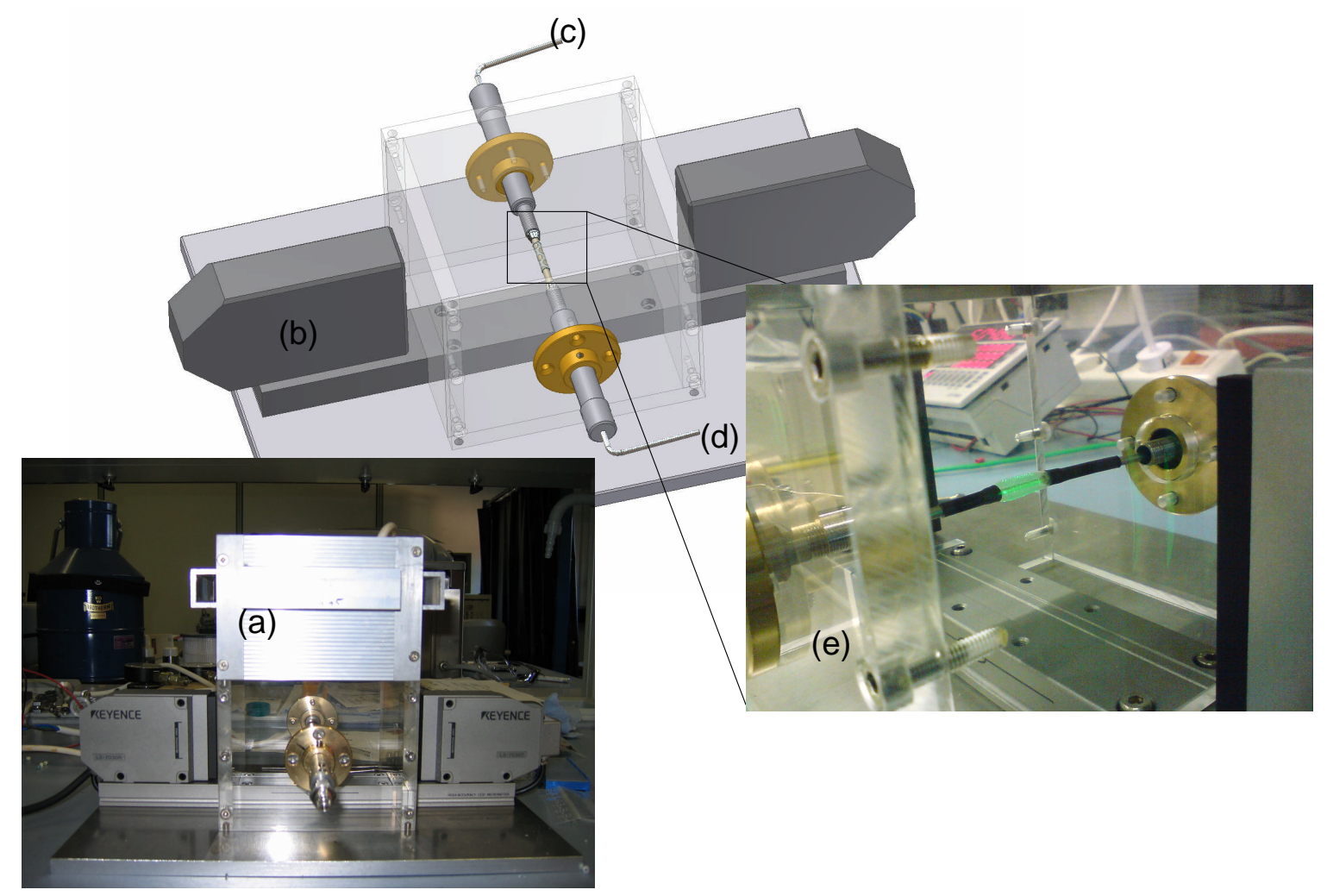

Figure 4.27: Radial force device components: (a) heating chamber, (b) optical micrometer from Keyence (LS series), (c) outlet tube - valve, (d) inlet tube - air pressure calibrator, (e) latex tube + stent.

The aim of hoop force characterization was to compare qualitatively a conventional TiNi stent and a TiNi thin film stent. Figure 4.28 shows a hoop force test on a conventional TiNi stent - $70 \mu m$ thickness.

At first, the hoop force of a thin walled silicon reference, $3.7 \mathrm{~mm}$ diameter and $0.3 \mathrm{~mm}$ thickness, as reference was measured at $36^{\circ} \mathrm{C}$. The reference curve shows a "superelastic" plateau, which was expected for a rubber material. After that a conventional TiNi stent, $3.5 \mathrm{~mm}$ diameter and $70 \mu \mathrm{m}$ thickness was placed over the same thin walled silicon and tested. The system (thin walled silicon + stent) hoop force increases in relation to the 


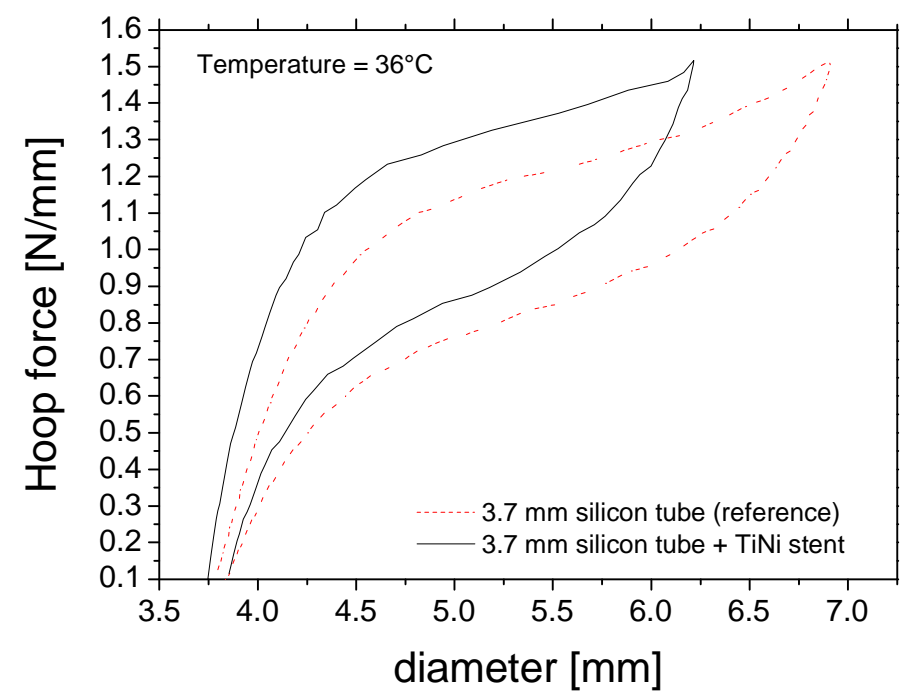

Figure 4.28: Hoop force test on a conventional TiNi stent - $70 \mu \mathrm{m}$ thickness. A thin walled silicon of $3.5 \mathrm{~mm}$ diameter and $0.3 \mathrm{~mm}$ thickness was used as reference.

reference and the superelastic plateau is still observed. The real stent hoop force is the subtraction between the reference and the measured stent hoop force curve.
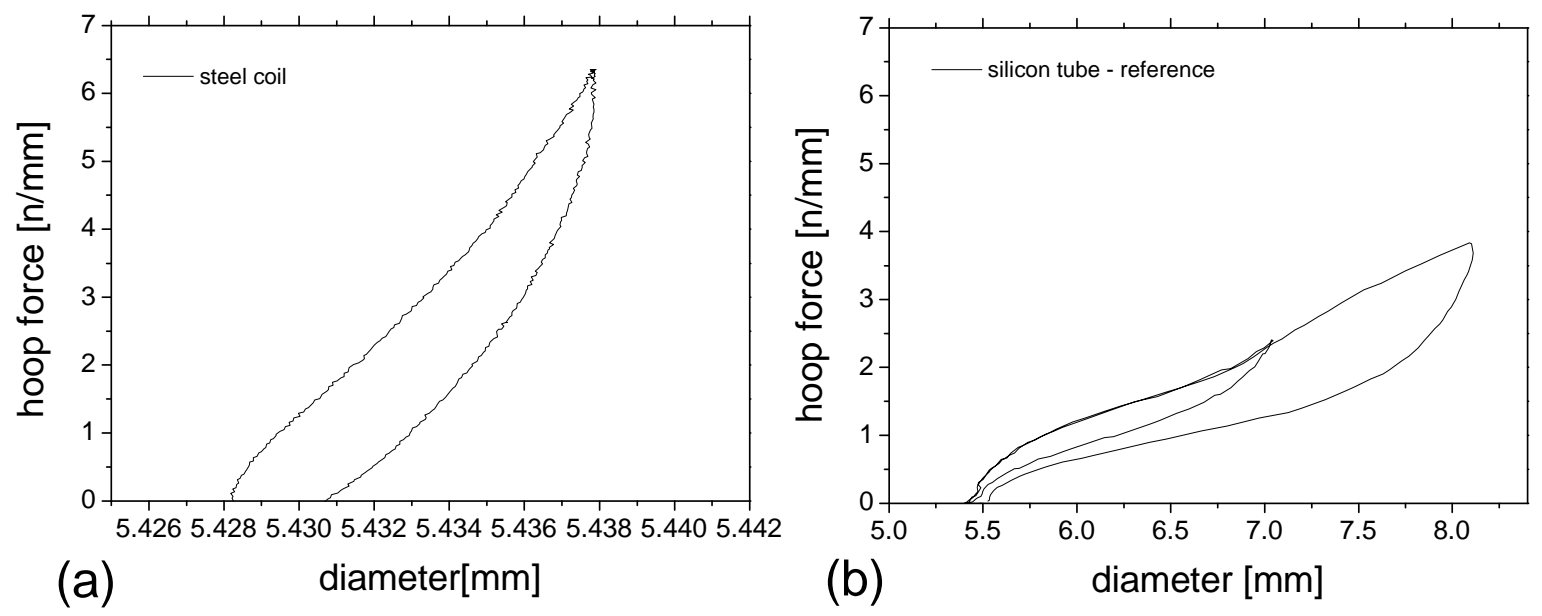

Figure 4.29: (a) Hoop force test on a steel coil and (b) Hoop force test on a silicon reference.

To be sure that the superelastic plateau is from the TiNi superelastic property, a hoop force measurement was realized on a steel coil, as shown in figure 4.29. The test carried 
out on a steel coil showed a higher hoop force, no plateau and a remmained plastic deformation, which validated the use of a thin walled silicon reference. A TiNi thin film stent of $5 \mu \mathrm{m}$ thickness was produced using the optimized parameters described in the section 4.2.1 and tested using the hoop force method. At first, the hoop force of a thin walled silicon (2.9 $\mathrm{mm}$ diameter and $0.1 \mathrm{~mm}$ thickness) was measured as reference at $36{ }^{\circ} \mathrm{C}$ and then a TiNi thin film stent, as shown in figure 4.30 .

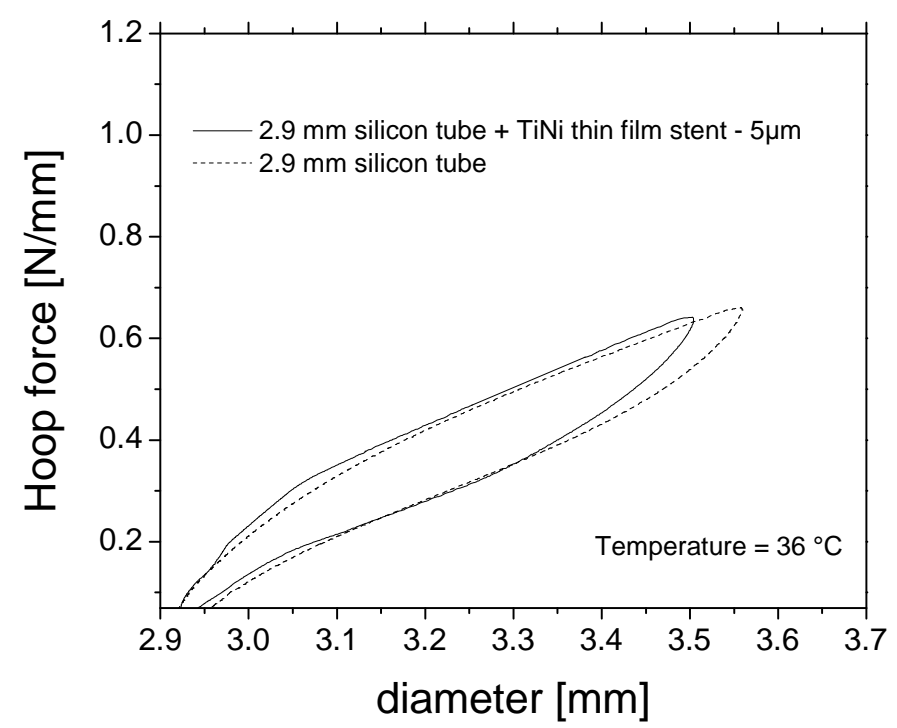

Figure 4.30: Hoop force test on a TiNi thin film stent. A thin walled silicon of $2.9 \mathrm{~mm}$ diameter and $0.1 \mathrm{~mm}$ thickness was used.

The real hoop force is smaller than the conventional stent, as expected, because of the difference on the stent thickness, but the superelastic effect still can be observed. The hoop force test method, developed in this research, demonstrated itself to be a qualitative tool to characterize TiNi thin film stents devices. 


\subsection{Structuring cylindrical TiNi thin films}

As shown in section 4.2 the use of magnetron sputtering together with the developed rotational device is capable of fabricating superelastic cylindrical TiNi thin films. This section will describe a technology, developed during this research, based on lithography and wet etching responsible for pattern cylindrical thin films.

\subsubsection{Cylindrical lithography}

Figure 4.31 shows the entire processes flow for fabrication of cylindrical structured TiNi thin films devices, from sputtering to wet etching: (1) cylindrical sputtering, (2) dip coating, (3) cylindrical lithography, (4) TiNi wet etching, (5) sacrificial layers wet etching. At first, a thin layer of an organic polymer (photoresist) is used to coat the surface of the cylindrical specimen by means of dip coating.

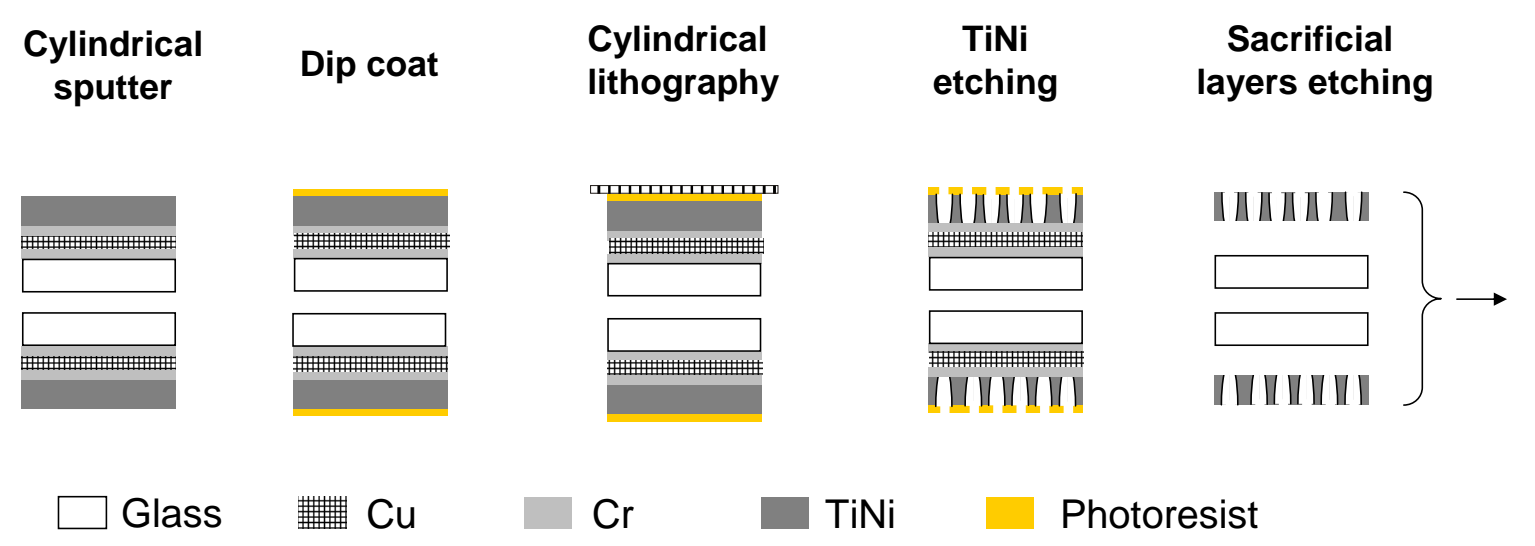

Figure 4.31: Process flow, from sputtering to wet etching: (1) cylindrical sputtering, (2) dip coating, (3) cylindrical lithography, (4) TiNi wet etching, (5) Sacrificial layers wet etching. 
The thickness $h$ of the dip coated photoresist, is a function of withdrawal velocity, photoresist viscosity and sample diameter, as described by Landau, Levich and Derjaguin [38],

$$
h \approx r \cdot\left(\frac{\eta \cdot V}{\gamma}\right)^{\frac{2}{3}}
$$

where $\eta$ is the liquid viscosity, $\gamma$ the surface tension, $V$ the withdrawal velocity and $r$ cylindrical specimen radius. Figure 4.32 shows the principle of the Landau, Levich and Derjaguin (LLD) theory. The region where the film is formed is called meniscus, with thickness $h$ and length $\lambda$.

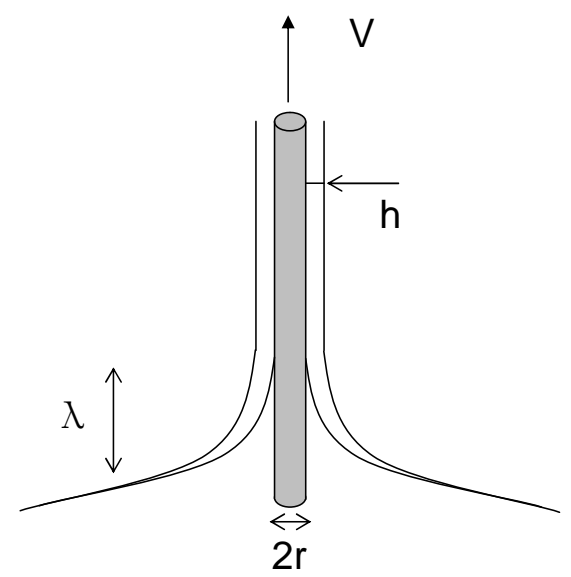

Figure 4.32: LLD fluid coating theory on a cylindrical specimen.

The photoresist thickness and its homogeneity are very important for the final feature resolution of the lithography process as described in section 2.4.2. Thus, photoresist thickness and its homogeneity were analyzed. For cylindrical lithography method a positive photoresist, AZ1518, from the company Microchemicals was used. The viscosity of this photoresist at $25^{\circ} \mathrm{C}$ is $34.2 \times 10^{-6} \mathrm{~m}^{2} / \mathrm{s}$ and its sensitivity is $55 \mathrm{~mJ} / \mathrm{cm}^{2}$ per $2 \mu \mathrm{m}$ photoresist thickness. Figure 4.33 shows the influence of the substrate withdrawal rate on the photoresist thickness in two substrates with diameter $3.0 \mathrm{~mm}$ (a) and $1.5 \mathrm{~mm}$ (b). The error bars correspond to the standard deviation from the thickness homogeneity along the substrate, figure 4.34 . 


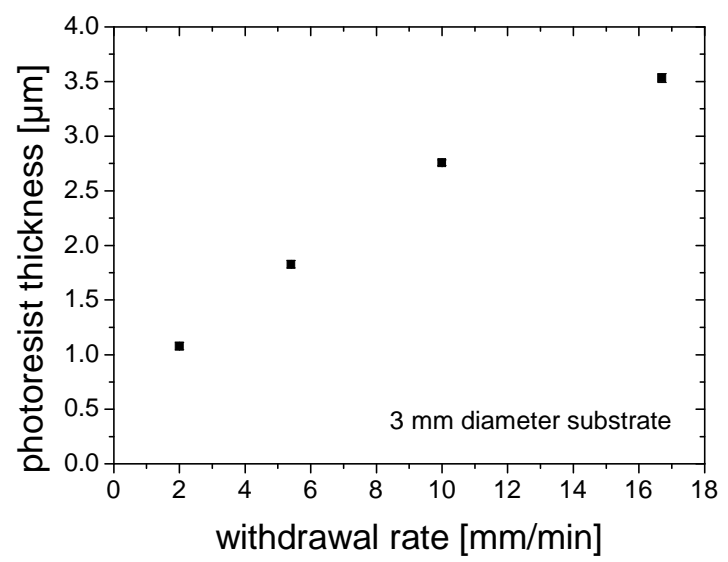

(a)

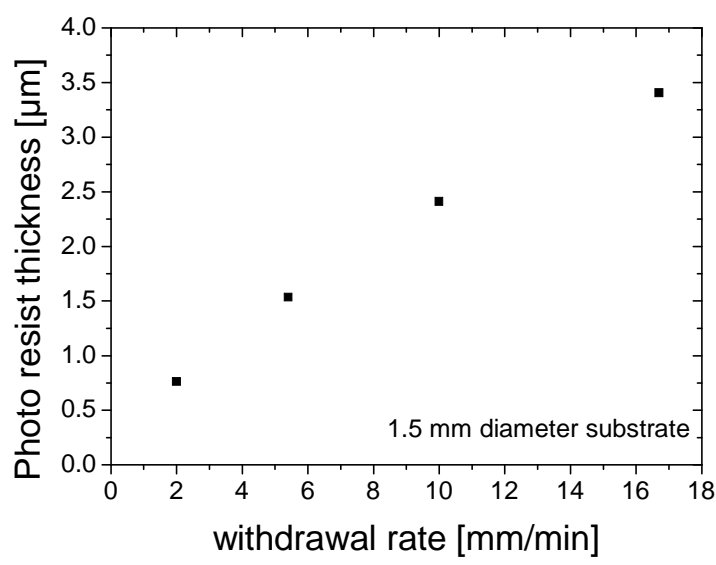

(b)

Figure 4.33: Influence of the substrate withdrawal rate on the photoresist thickness in two substrates with diameter 3.0 (a) and $1.5 \mathrm{~mm}$ (b).

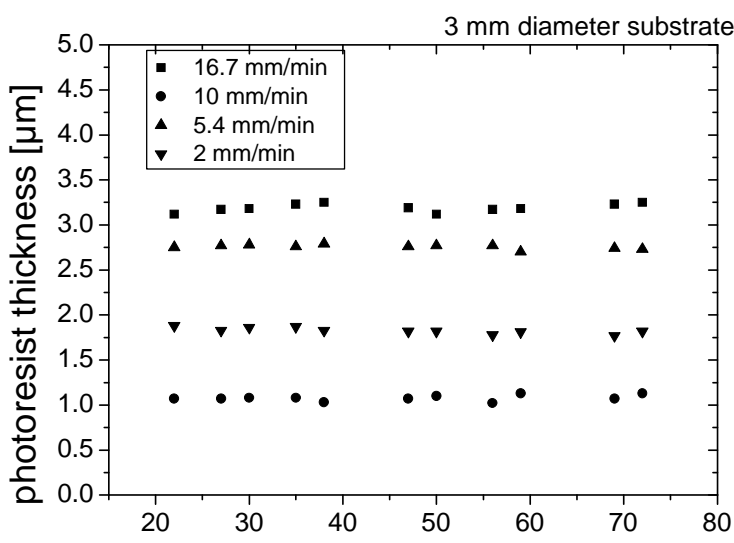

(a)

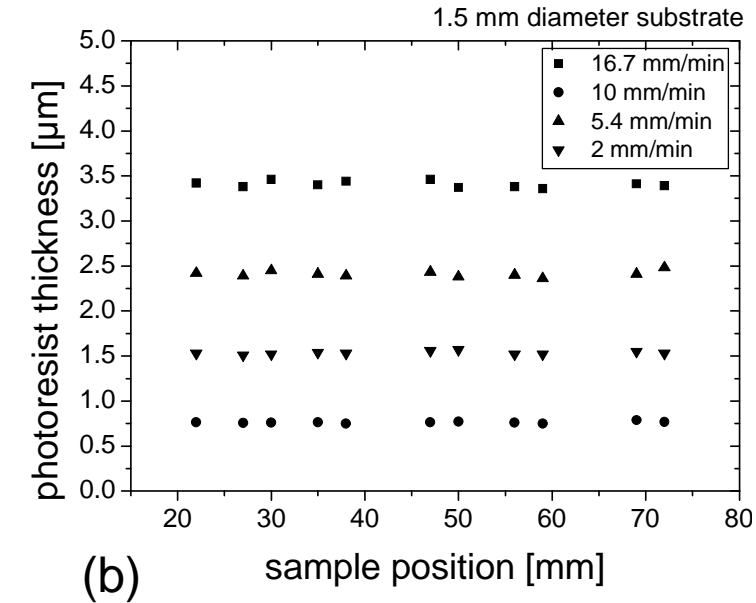

(b)

Figure 4.34: Thickness homogeneity as a function of withdrawal rate and substrate diameter. (a) $3 \mathrm{~mm}$ diameter substrate (b) $1.5 \mathrm{~mm}$ diameter

The photoresist showed thickness variation smaller than $0.5 \%$ along the sample in all withdrawal speed and for both substrates. For the further development of cylindrical lithography a withdrawal rate of $5.4 \mathrm{~mm} / \mathrm{min}$ and a soft bake of 8 minutes at $105{ }^{\circ} \mathrm{C}$ were set, based on the previous results 4.33 (a) and (b).

Cylindrical lithography is based on the synchronization of the planar mask with the rotation of the cylindrical substrate as sketched in figure 4.35 .

To achieve a high resolution a slit aperture (shutter) is used so that only the part 


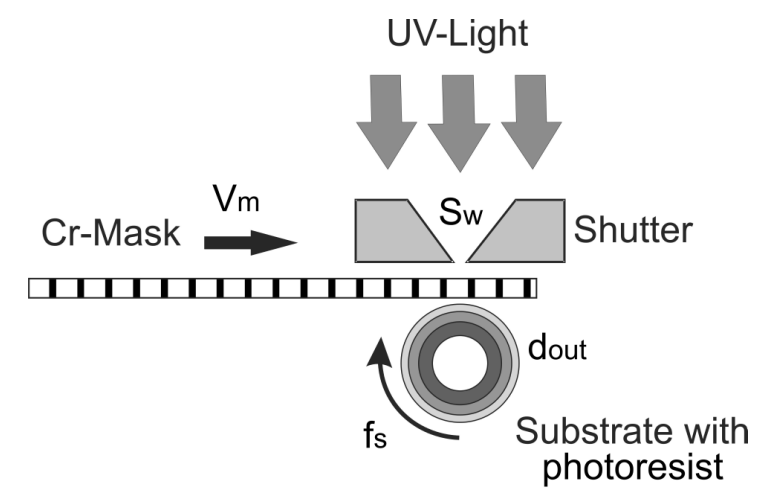

Figure 4.35: Basic principle of cylindrical UV lithography. Where $V_{m}$ is the $\mathrm{Cr}$ mask lateral speed, $S_{w}$ the width of the slit aperture, $f_{s}$ the rotational frequency of the cylindrical substrate and $d_{\text {out }}$ the outer diameter of the cylindrical sample.

of the cylinder facing toward the Cr mask is exposed to UV light. Critical parameters are the lateral speed of the Cr mask $\left(V_{m}\right)$, the rotational frequency of the cylindrical substrate $\left(f_{s}\right)$, and the width of the slit aperture $\left(S_{w}\right)$. A set of optimum parameters has to be adjusted for each different outer diameter of the cylindrical sample $\left(d_{\text {out }}\right)$, taking into account the required exposure time $\left(t_{e}\right)$ of the individual photoresist. These synchronized movements can be set up using the following equation:

$$
V_{m}=\frac{S_{w}}{t_{e}}
$$

To keep the movement of the cylindrical substrate and the Cr mask synchronized, the substrate has to rotate with the following frequency $f_{s}$ :

$$
f_{s}=\frac{V_{m}}{\pi d_{\text {out }}}=\frac{S_{w}}{\pi d_{\text {out }} t_{e}}
$$

To realize this set-up a standard Suess (MJB4) Microtec Lithography device was adapted in such a way that it can be operated in combination with a self-developed rotational device shown in figure 4.36. The rotational device was constructed in order 
to expose substrates from 0.5 to $5 \mathrm{~mm}$ diameter using two linear motors, a rotational one with a $0.01^{\circ}$ resolution and a linear one with a $6.3 \mu \mathrm{m}$ resolution mounted on a $\mathrm{x}-\mathrm{y}$ linear table.

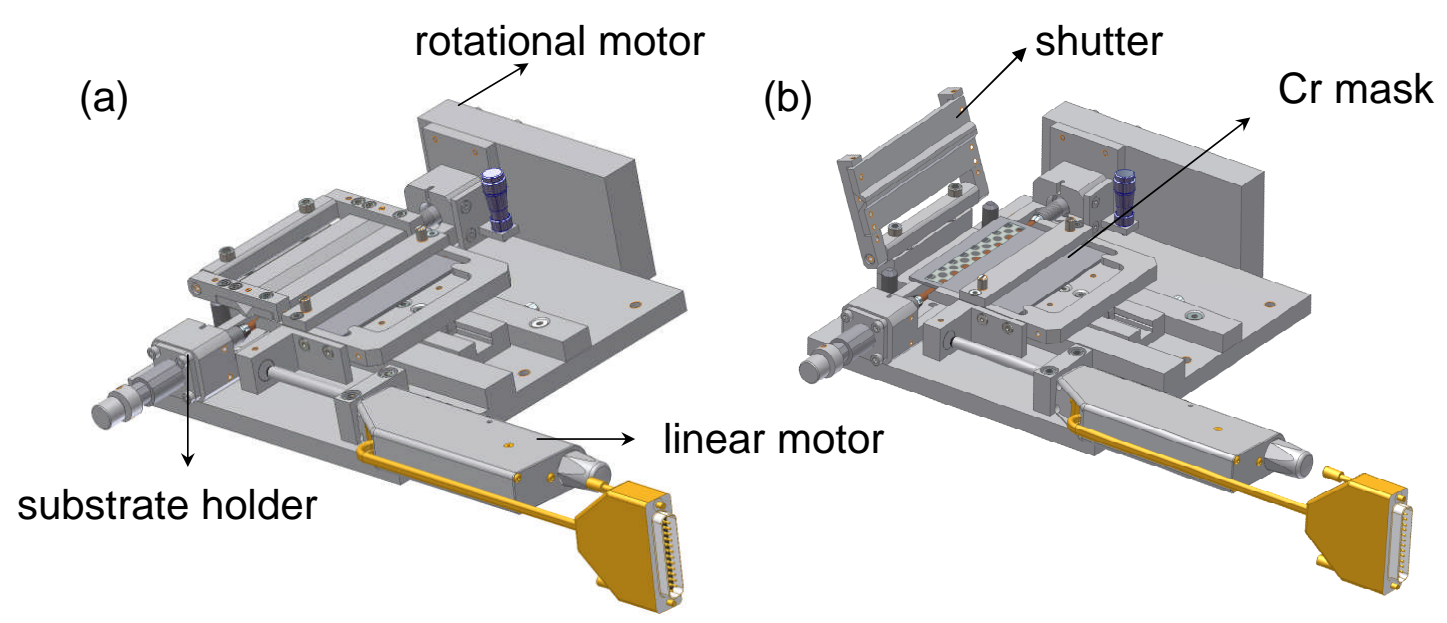

Figure 4.36: Engineering sketch of the rotational device for cylindrical UV lithography. (a) closed shutter b) open shutter .

A substrate holder allows a vertical adjustment of the cylindrical substrate in relation to the $\mathrm{Cr}$ mask with a precision of $1 \mu \mathrm{m}$. This vertical adjustment allows the exposure of substrates with different diameters and is an important parameter to define the feature size resolution. Further parameters are the UV wavelength and optical properties. Figure 4.37 shows the cylindrical UV lithography device mounted on the Suess (MJB4) Microtec Lithography device.

As described in section 2.4.2, the resist feature size resolution $(W)$, according to the near field or Fresnel diffraction, is defined by:

$$
W=(\lambda g)^{1 / 2}
$$

where $\lambda$ is the wavelength and $g$ the gap between resist surface and Cr mask. For cylindrical lithography a soft mode contact due to the dynamic movement between $\mathrm{Cr}$ mask and substrate is adjusted. 


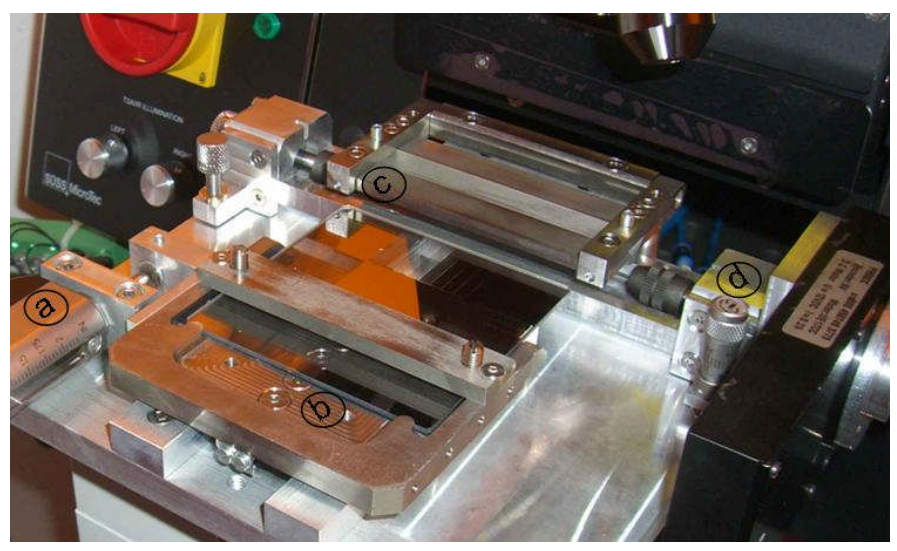

Figure 4.37: Cylindrical UV lithography device mounted on the Suess (MJB4) Microtec Lithography device. (a) corresponds to the linear horizontal motor, (b) to the Cr mask, (c) to the shutter, and (d) to the rotational motor.

The feature size resolution study was realized comparing the resolution of standard planar UV lithography with circular UV lithography. For this comparison a planar TiNi thin film with $2 \mu m$ thickness was deposited on a 4 inch Si wafer with the parameters described in section 4.1. After the sputtering process, the film was spin coated with photoresist AZ1518 of $1.5 \mu \mathrm{m}$ thickness. The soft bake was performed on a hotplate at $105{ }^{\circ} \mathrm{C}$ for 2 minutes followed by rehydration in a controlled atmosphere for another 2 minutes. A chromium covered quartz mask with a feature resolution of $\pm 500 \mathrm{~nm}$ was used for the soft contact photolithography. The exposure time was $2 \mathrm{~s}$ and the resist was developed using a AZ716 MIF solution for $30 \mathrm{~s}$. Cylindrical thin films were sputtered on a glass tube of $1.5 \mathrm{~mm}$ outer diameter by employing the rotational device. Deposition parameters are described in section 4.2.1. The cylindrical sample was dip coated and a withdrawal rate of $5.4 \mathrm{~mm} / \mathrm{min}$ was chosen in order to obtain a constant photoresist thickness of $1.5 \mu \mathrm{m}$ throughout the sample, as shown figure 4.34. The cylindrical sample was soft baked at $105^{\circ} \mathrm{C}$ for 8 minutes and afterwards it was rehydrated in a controlled atmosphere for another 2 minutes. The lateral speed of the $\mathrm{Cr}$ mask $\left(V_{m}\right)$ used was $0.025 \mathrm{~mm} / \mathrm{s}$, the rotational frequency of the cylindrical substrate $\left(f_{s}\right)$ used was $1.8181^{\circ} / \mathrm{s}$, and the width of the slit aperture $\left(S_{w}\right)$ was $50 \mu \mathrm{m}$. The exposure time was 
$2 \mathrm{~s}$ and the photoresist was developed using a AZ716 MIF solution for $30 \mathrm{~s}$. For the resolution comparison a $\mathrm{Cr}$ mask containing circular patterns with different diameters was used. An example of this pattern is shown in figure 4.38 (a) and (b). The feature size varies between 5 and $60 \mu \mathrm{m}$.

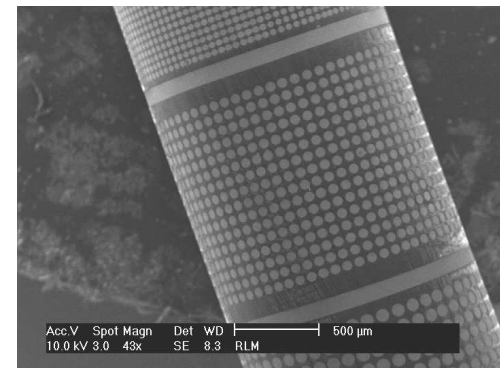

(a)

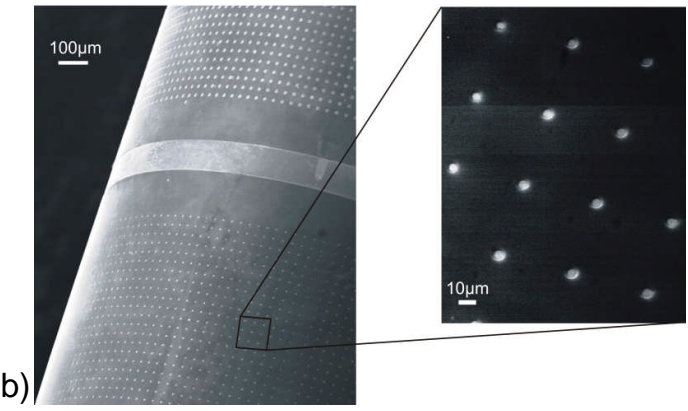

Figure 4.38: Example of a photoresist patterning. The scanning electron microscope images show circular patterns with feature sizes ranging from 30 $\mu \mathrm{m}$ to $60 \mu \mathrm{m}$ diameter (a) and from $5 \mu \mathrm{m}$ to $10 \mu \mathrm{m}$ (b).

Figure 4.39 shows two light-optical micrographs, which were overlapped for better comparison. Figure 4.39 (a) represents the pattern of a circular substrate $(1.5 \mathrm{~mm}$ diameter) and (b) the pattern of a planar substrate, both with a feature size of $5 \mu \mathrm{m}$. No significant difference between the patterned geometries can be observed.

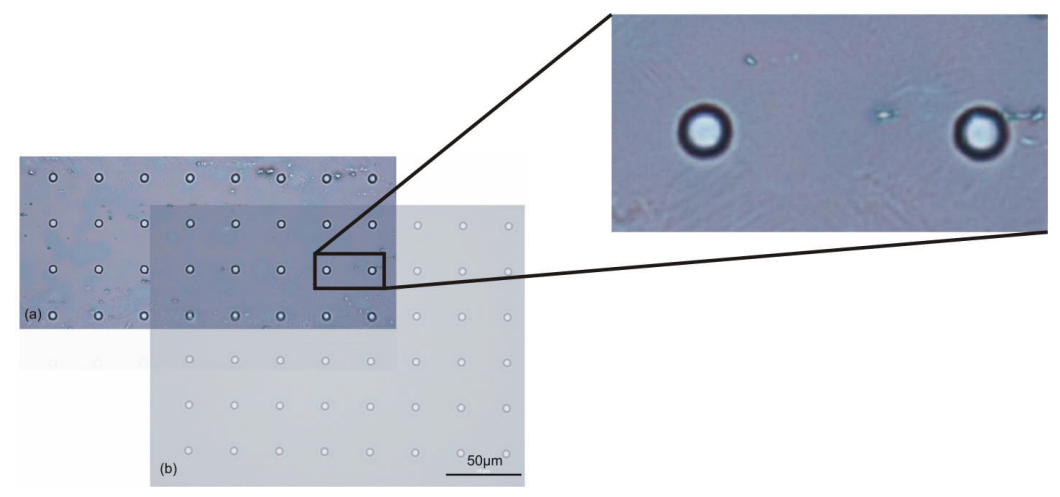

Figure 4.39: Photoresist patterns with feature sizes of $5 \mu m(a)$ in a cylindrical sample of $1.5 \mu \mathrm{m}$ diameter and (b) in a planar sample.

Eleven internal diameters of patterned samples (planar/cylindrical) were measured and compared, as presented in Table 4.3 . 
Table 4.3: Feature size comparison of cylindrical and planar substrates

\begin{tabular}{cccc}
\hline $\begin{array}{c}\text { Geometry } \\
\text { Nr. }\end{array}$ & $\begin{array}{c}\text { Cr Mask } \\
{[\mu m]}\end{array}$ & $\begin{array}{c}\text { Cylindrical } \\
{[\mu m]}\end{array}$ & $\begin{array}{c}\text { Planar } \\
{[\mu m]}\end{array}$ \\
\hline \hline 1 & 5 & 4.8 & 4.6 \\
2 & 10 & 9.8 & 9.2 \\
3 & 20 & 18.5 & 19.1 \\
4 & 40 & 37.9 & 38.9 \\
5 & 60 & 58.9 & 60.2 \\
6 & 80 & 79.4 & 79.8 \\
7 & 100 & 100.1 & 99.5 \\
8 & 140 & 141.3 & 140.7 \\
9 & 180 & 181.9 & 180.9 \\
10 & 200 & 201.2 & 201.7 \\
11 & 400 & 402.9 & 402.0 \\
\hline \hline
\end{tabular}

For all analyzed geometries - the patterning of the cylindrical substrates shows a lateral resolution comparable to the planar patterning.

Mismatch resolution in two directions, tangential $\left(\Delta X_{N}\right)$ and axial $(\Delta y)$, was also analyzed, as shown in Fig. 4.40 .

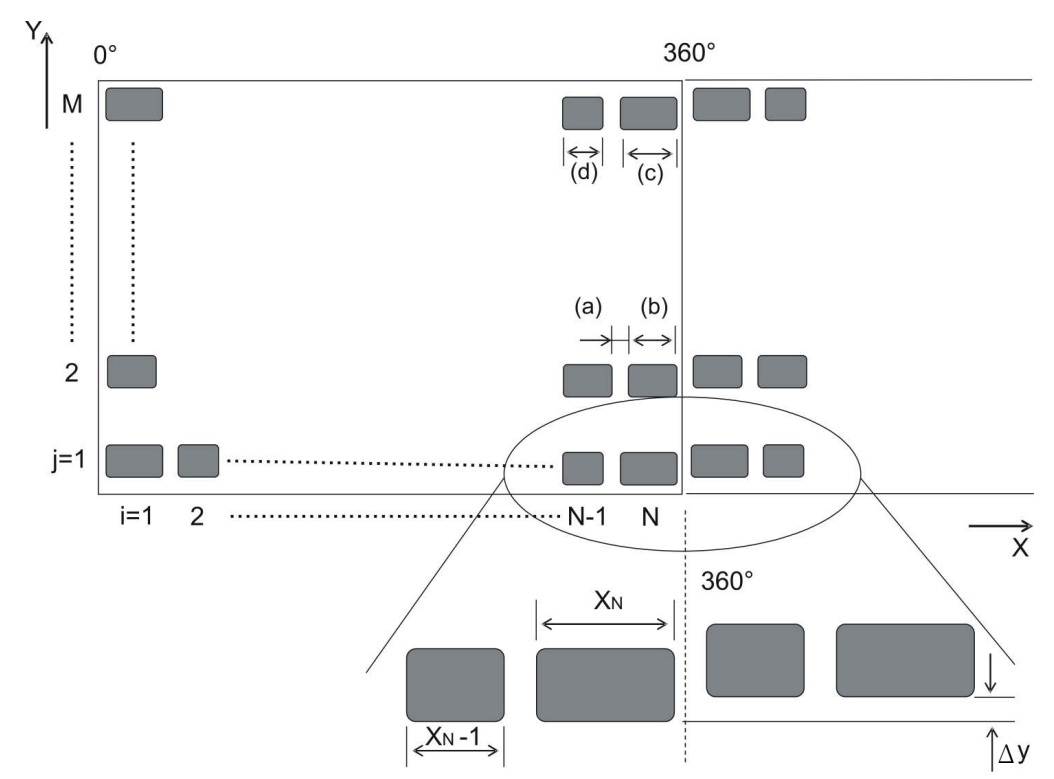

Figure 4.40: Tangential $\left(\Delta X_{N}\right)$ and axial $(\Delta y)$ mismatch resolution. 
Three errors were observed: angular misalignment,

$$
<\Delta y>_{M}
$$

rotational frequency of the cylindrical substrate $\left(f_{s}\right)$ misalignment,

$$
<\Delta X(i=1, \ldots N-1)>-X_{\text {Mask }}
$$

and the substrate radial tolerance,

$$
<\Delta X_{N}-\Delta X(i=1, \ldots N-1)>
$$

To understand these mismatchs a Cr mask was developed to be used on a $3.5 \mathrm{~mm}$ diameter substrate, with a circular measurement $(2 \pi r)$ equal to $10.99 \mathrm{~mm}$. The substrate had a diameter tolerance of $\pm 0.1 \mathrm{~mm}$. A starting point was patterned to control the overlap region. The distance between two geometries, figure 4.40 (a), is constant and all geometries are aligned vertically as presented in figure 4.40. As illustrated in table 4.4 measurements using an optical microscope were taken in order to compare the feature size between the Cr mask and the feature size achieved after the exposure. Two regions of the sample were chosen, one at 90 degrees and the second at 360 degrees.

Table 4.4: Feature size comparison of two sample regions: at 360 and 90 degree.

\begin{tabular}{|l|c|c|}
\hline Geometry from figure 4.38 & b & c \\
\hline \hline Cr mask $[\mu \mathrm{m}]$ & 510 & 590 \\
\hline \hline Measured structured photoresist at 90 degree $[\mu \mathrm{m}]$ & 510 & 595 \\
\hline Measured structured photoresist at 360 degree $[\mu \mathrm{m}]$ & 449 & 529 \\
\hline
\end{tabular}

Thus, the tangential resolution $\left(\Delta X_{N}\right)$ in this example is $61 \mu m$ which is mainly related to the radial tolerance and to some extent to the resolution of the linear motor 
$(6.3 \mu \mathrm{m})$. Fig. 4.41 shows a Scanning Electron Microscope image of an overlapped patterning on a $3.5 \mathrm{~mm}$ diameter substrate.

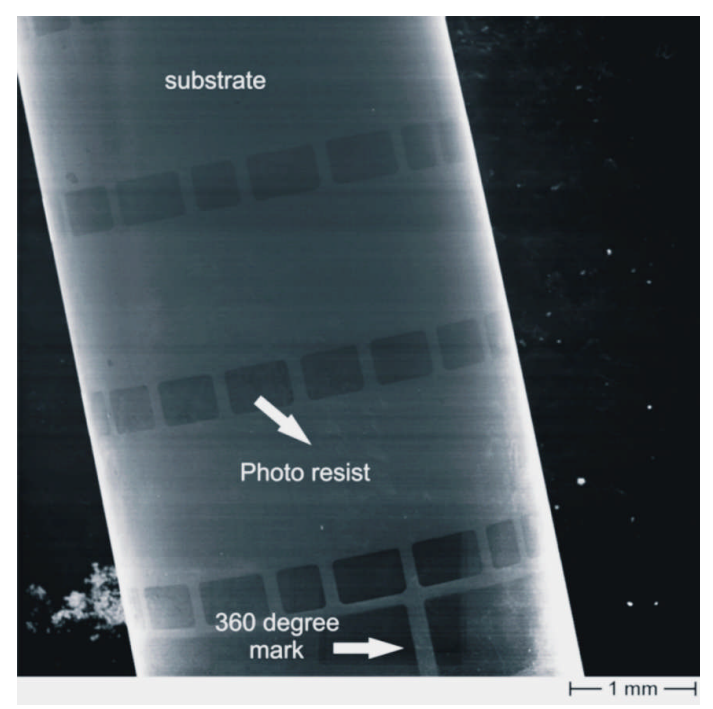

Figure 4.41: SEM image of an overlapping pattern on a $3.5 \mathrm{~mm}$ diameter substrate.

The distance between the geometry (figure 4.40 (a)) at 360 degrees and 90 degrees was measured using a surface profilometer. The results are presented in figure 4.42 .

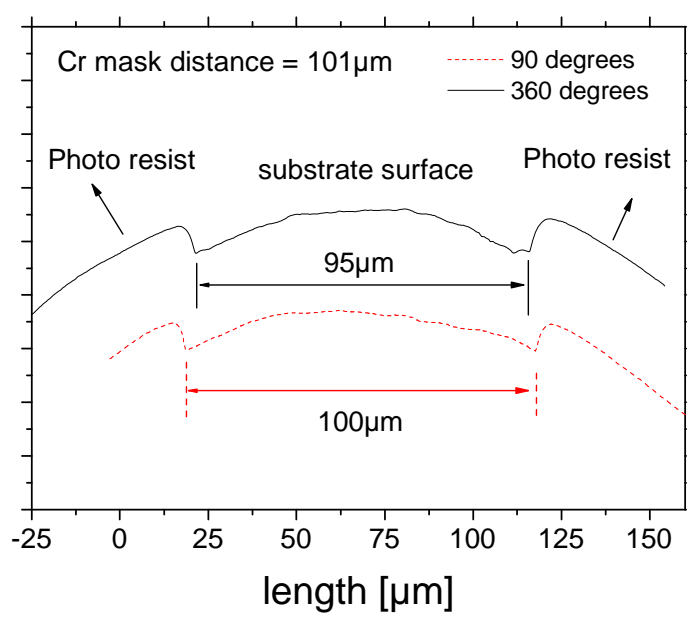

Figure 4.42: Profilometer measurements: Comparison of the distance between two geometries at 360 degree and 90 degree.

Figure 4.43 shows a $3 \mu \mathrm{m}$ axial mismatch of an overlapped exposed sample. 


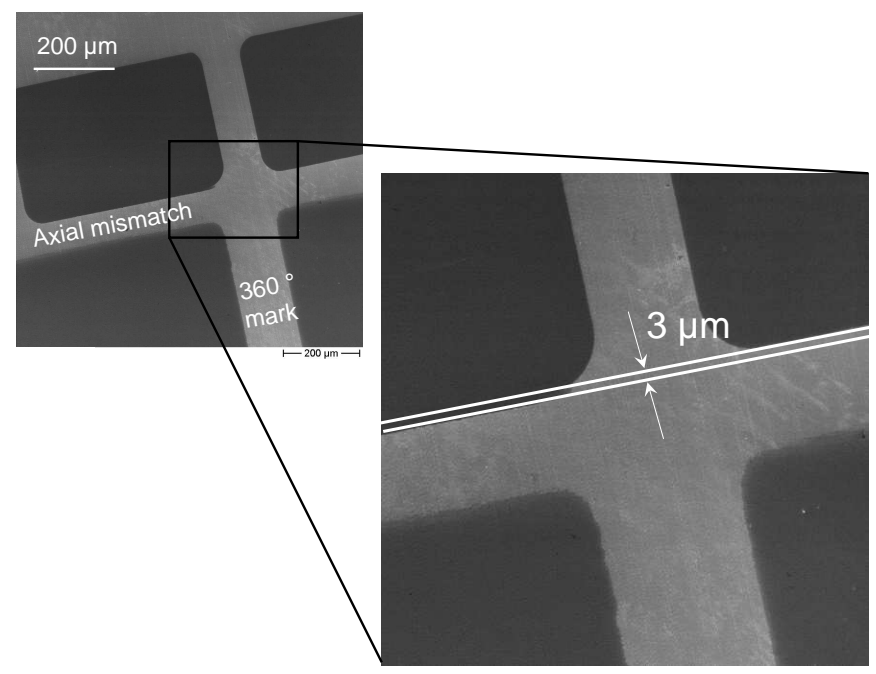

Figure 4.43: SEM image of an axial mismatch of an overlapped exposed sample.

We have developed a rotational UV lithography device based on the standard photo lithography process to pattern cylindrical samples of different diameters and lengths with a feature resolution down to $5 \mu \mathrm{m}$ and an axial resolution $(\Delta y)$ of $3 \mu \mathrm{m}$. The axial resolution mainly depends on the alignment between Cr mask and sample and can be enhanced by placing a reference mark on the $\mathrm{Cr}$ mask. The tangential resolution $\left(\Delta X_{N}=61 \mu \mathrm{m}\right)$ is affected by the substrate radial tolerance and the linear motor tolerance. The resulting divergence can be minimized using a substrate with a small radial tolerance and a motor with a precision better than $6.3 \mu \mathrm{m}$. The developed rotational UV lithography device allows the patterning of complex geometries on the surface of a cylindrical sample and was successfully employed to pattern TiNi thin film tubes as shown in figure 4.44 .

\subsubsection{Wet etching}

After the photoresist exposure the film is wet etched. The wet chemical etching process is an isotropic process, as described in section 2.4.3, which causes some design limitation in relation to the desired thickness and feature widths. The optimization of the etchant 


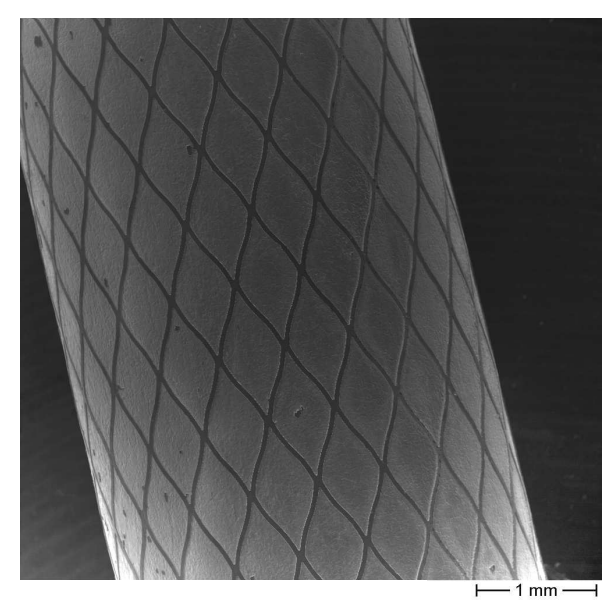

Figure 4.44: SEM image of stent like structure patterned with cylindrical UV lithography.

and the film morphology are basically very important in reducing the isotropic behavior thereby increasing the etching resolution.

This section will present results of the influence of etching parameters: movement of the etchant and etchant composition on the etching rate and lateral ratio. As described in section 2.4.3, the lateral ratio is the relation between horizontal etching rate $\left(\dot{E}_{h}\right)$ and the vertical etching rate $\left(\dot{E}_{v}\right)$. The ratio for a complete isotropic process is 1 . The wet etching optimization towards a partially anisotropic behavior was performed using sputtered planar amorphous TiNi thin films. The films were sputtered on $30 \mathrm{x}$ $30 \mathrm{~mm}$ silicon wafer. The deposition parameters were identical for all samples. A Cr mask with a stent like geometry, as shown figure 4.45 was used and a profilometer 3.1.5 was used to measure the feature size and the etching rate.

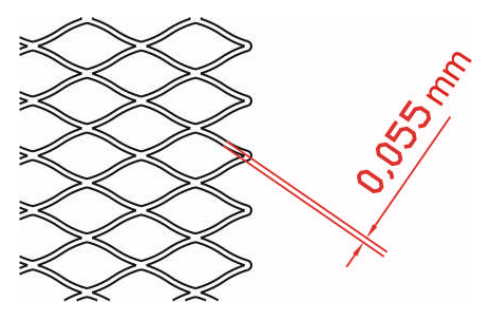

Figure 4.45: Generic stent design. 
The influence of the etchant movement on the etching rate was analyzed. A starting etchant composition was used based on previous results [89] [90]: $\mathrm{H}_{2} \mathrm{O}(490 \mathrm{ml}), \mathrm{HF}$ $(26.4 \mathrm{ml}), \mathrm{HNO}_{3}(82.8 \mathrm{ml})$ and $\mathrm{NiSO}_{4}(30 \mathrm{~g})$. Two wet etching experiments were performed: using a magnetic stirring to move the etchant and without magnetic stirring. Figure 4.46 (a) shows the depth as a function of etching time.
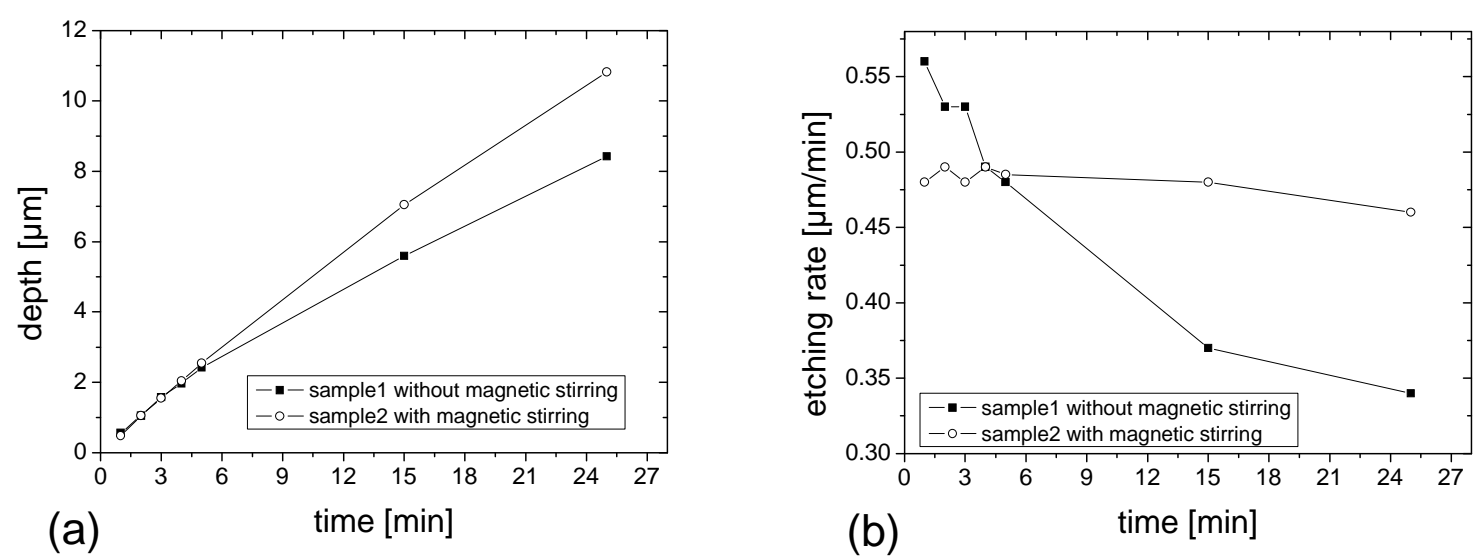

Figure 4.46: (a) Depth profile as a function of etching time, (b) etching rate as a function of time. Sample 1 wet etched without stirring, sample 2 wet etched with magnetic stirring.

The sample 1 etched without a magnetic stirring shows lower depth than the sample 2 etched with a magnetic stirring, consequently an inhomogeneous etching rate along the time, as shown in figure 4.46 (b). The reason for this effect, as described in 2.4 .3 is the movement of the etchant which allows the transport of the reaction products away from the surface of the specimen.

In a first experiment, the composition of the etchant was varied with respect to the HF concentration. The total amount of etchant was reduced to $40 \mathrm{ml}$ and the concentration of $\mathrm{H}_{2} \mathrm{O}, \mathrm{HNO}_{3}$ and $\mathrm{NiSO}_{4}$ was kept constant: $81.7 \mathrm{ml}, 13.8 \mathrm{ml}$ and $4 \mathrm{~g}$ respectively. The influence of the $\mathbf{H F}$ concentration on the etching rate was analyzed. The HF concentration was varied in five steps: $2 \mathrm{ml}, 3 \mathrm{ml}, 4.4 \mathrm{ml}$ (start solution), $5 \mathrm{ml}$ and 6 $\mathrm{ml}$ and the depth was measured, using profilometer, three times every 5 minutes. A magnetic stirring was used. Figure 4.47 (a) shows the depth as a function of etching time in all five concentrations. 

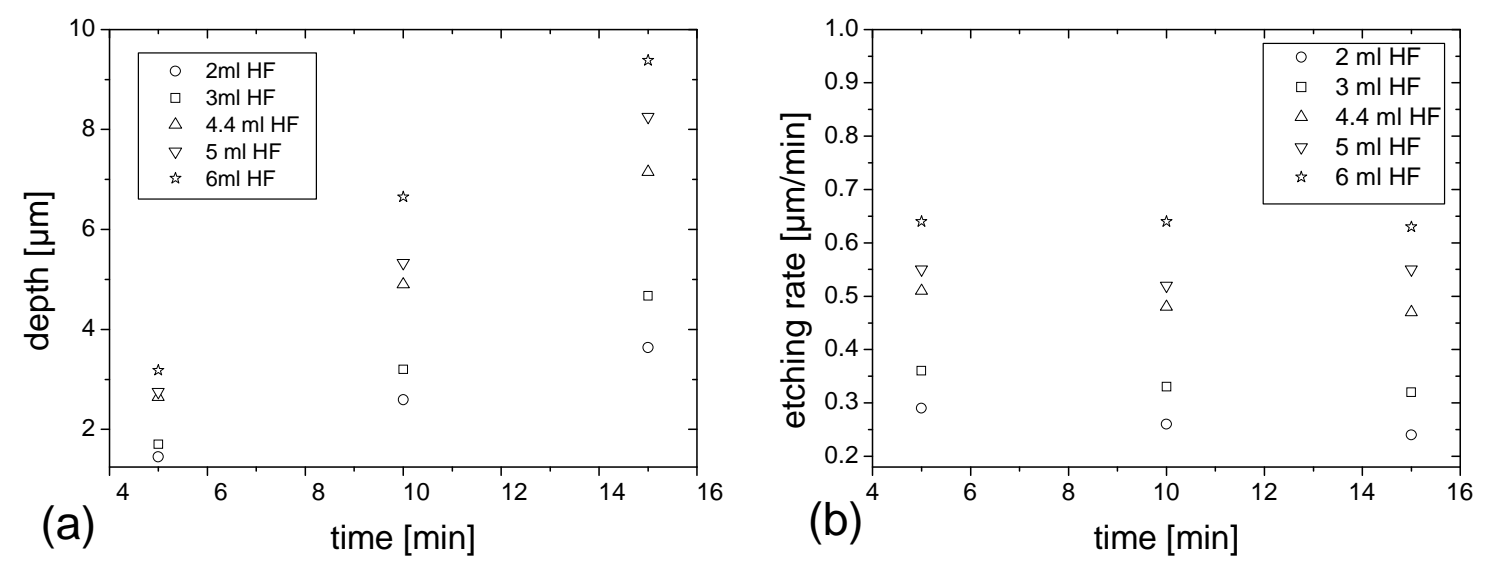

Figure 4.47: (a) Depth as a function of etching time, (b) etching rate as a function of time HF concentrations steps variation: $2 \mathrm{ml}, 3 \mathrm{ml}, 4.4 \mathrm{ml}$ (start solution), $5 \mathrm{ml}$ and $6 \mathrm{ml}$.

All five compositions showed a "linear function" of the depth profile in relation to the etching time. Increasing HF concentration enhances the depth profile and consequently the etching rate, as shown figure 4.47 (b). The influence of the HF concentration on the lateral ratio was analyzed. To calculate the lateral ratio, the feature size was measured, subtracted from the original size and then divided by the depth. This procedure was conducted three times for each depth. The error bars are the standard deviation of the profilometer measurements. Figure 4.48 shows a lateral ratio as a function of the HF concentration. The start solution $(4.4 \mathrm{ml} \mathrm{HF})$ showed a lateral ratio of 0.87 , while the etchant with $6 \mathrm{ml} \mathrm{HF}$ showed 0.78. The decrease of the lateral ratio with the increase of the HF concentration is associated with the bathing time. Reducing the bathing time will reduce the etching of material under the photoresist mask.

Using a fixed $6 \mathrm{ml} \mathrm{HF}$ as standard concentration, in a second optimization series the concentration of $\mathrm{NiSO}_{4}$ was varied. Figure 4.49 (a) shows the depth as a function of etching time in all five $\mathrm{NiSO}_{4}$ concentrations.

It was observed that increasing $\mathrm{NiSO}_{4}$ concentration reduces the depth and consequently the etching rate, as shown in figure 4.49 (b). The influence of the $\mathrm{NiSO}_{4}$ concentration on the lateral ratio was analyzed. Figure 4.50 shows a lateral ratio as a 


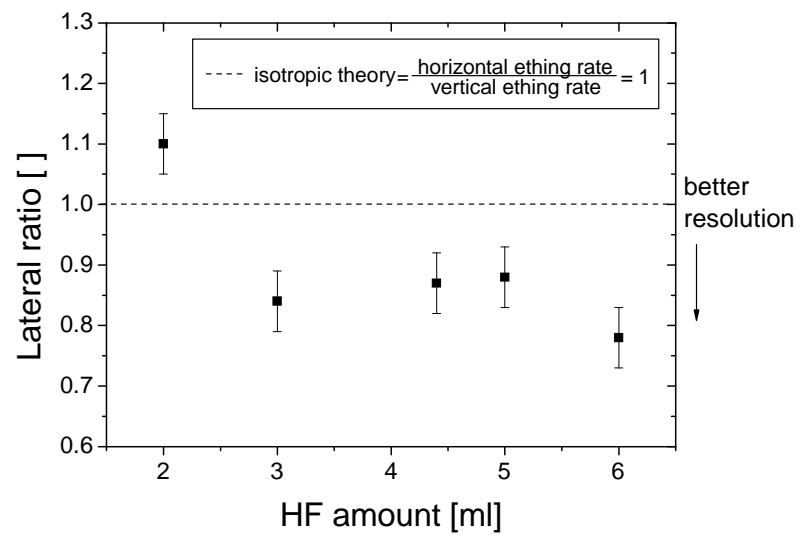

Figure 4.48: Influence of the HF concentration on the lateral ratio as a function of time. HF concentrations steps variation: $2 \mathrm{ml}, 3 \mathrm{ml}, 4.4 \mathrm{ml}$ (start solution), $5 \mathrm{ml}$ and $6 \mathrm{ml}$.
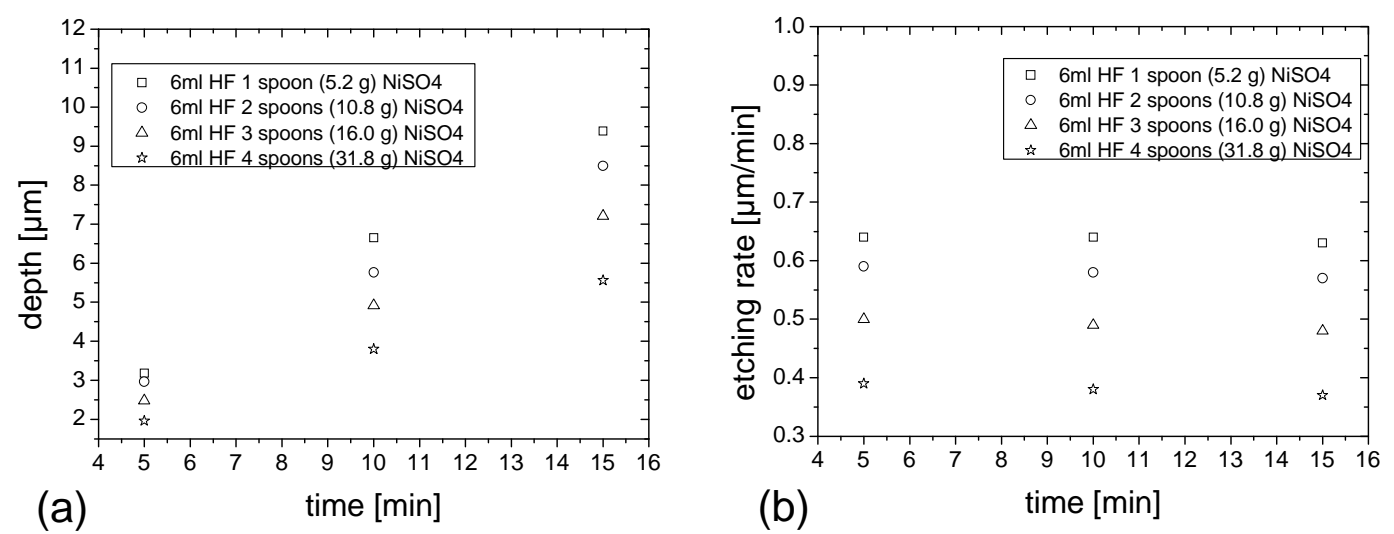

Figure 4.49: (a) Depth as a function of etching time, (b) etching rate as a function of time. $\mathrm{NiSO}_{4}$ concentrations steps variation: $5 \mathrm{~g}$ (start concentration), $10 \mathrm{~g}, 15 \mathrm{~g}$ and $20 \mathrm{~g}$.

function of the $\mathrm{NiSO}_{4}$ concentration.

The same effect as for the HF optimization was observed in the variation of the $\mathrm{NiSO}_{4}$ concentration in the etchant solution. With the enhancement of the concentration the etching rate reduces, figure 4.50. Thus, the sample has to be emerged in the bath for a long time allowing etching of the material under the photoresist mask. Table 4.5 shows the optimized TiNi etchant solution $(100 \mathrm{ml})$ and its properties.

As mentioned before, the lateral ratio reduction of an isotropic etching will enhance the 


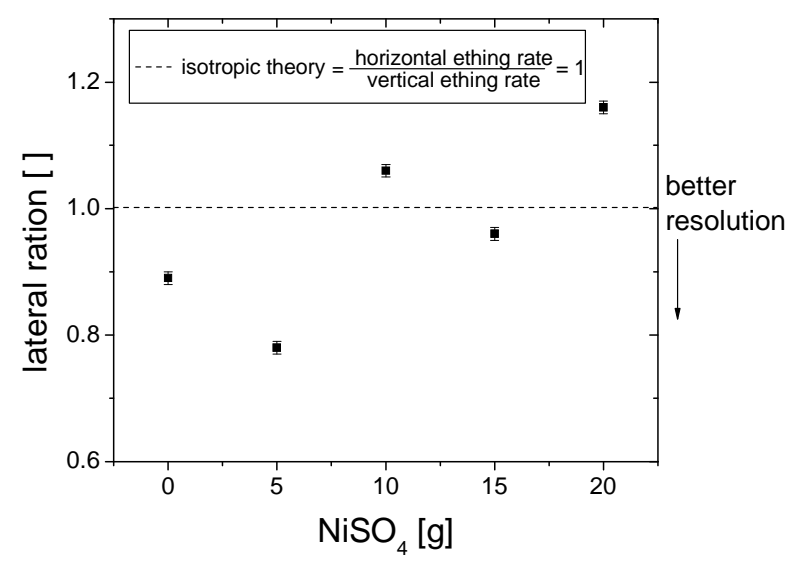

Figure 4.50: Influence of the $\mathrm{NiSO}_{4}$ concentration on the lateral ratio as a function of time. $\mathrm{NiSO}_{4}$ concentrations steps variation: $5 \mathrm{~g}$ (start concentration), $10 \mathrm{~g}, 15 \mathrm{~g}$ and $20 \mathrm{~g}$.

Table 4.5: Optimized TiNi etchant solution (100ml) and its properties.

\begin{tabular}{cccc}
\hline $\mathrm{H}_{2} \mathrm{O}$ & $\mathrm{HF}$ & $\mathrm{HNO}_{3}$ & $\mathrm{NiSO}_{4}$ \\
{$[\mathrm{ml}]$} & {$[\mathrm{ml}]$} & {$[\mathrm{ml}]$} & {$[\mathrm{g}]$} \\
\hline 80 & 6 & 14 & $\approx 5$ \\
\hline
\end{tabular}

etching rate: $0.64 \mu \mathrm{m}$

lateral ratio: $\quad 0.76$

resolution of the final device. Figure 4.51 shows a schematic comparison of the isotropic theory and our optimized TiNi etchant solution, where (a) is an optical micrograph from a photoresist structured with a stent like structure $55 \mu \mathrm{m}$ feature size, (b) is A-A system cross section, (c) the isotropic etching - final feature size $35.5 \mu \mathrm{m}$, and (d) the optimized solution - final feature size $40.3 \mu \mathrm{m}$.

The optimized etchant solution for planar films was used for cylindrical TiNi thin films. The same effect as for the planar film was observed when the etchant solution was not stirred. In addition to the reduction of etching rate and strong under etching, etching inhomogeneity along the sample was also observed. Thus, an etching rotational device 


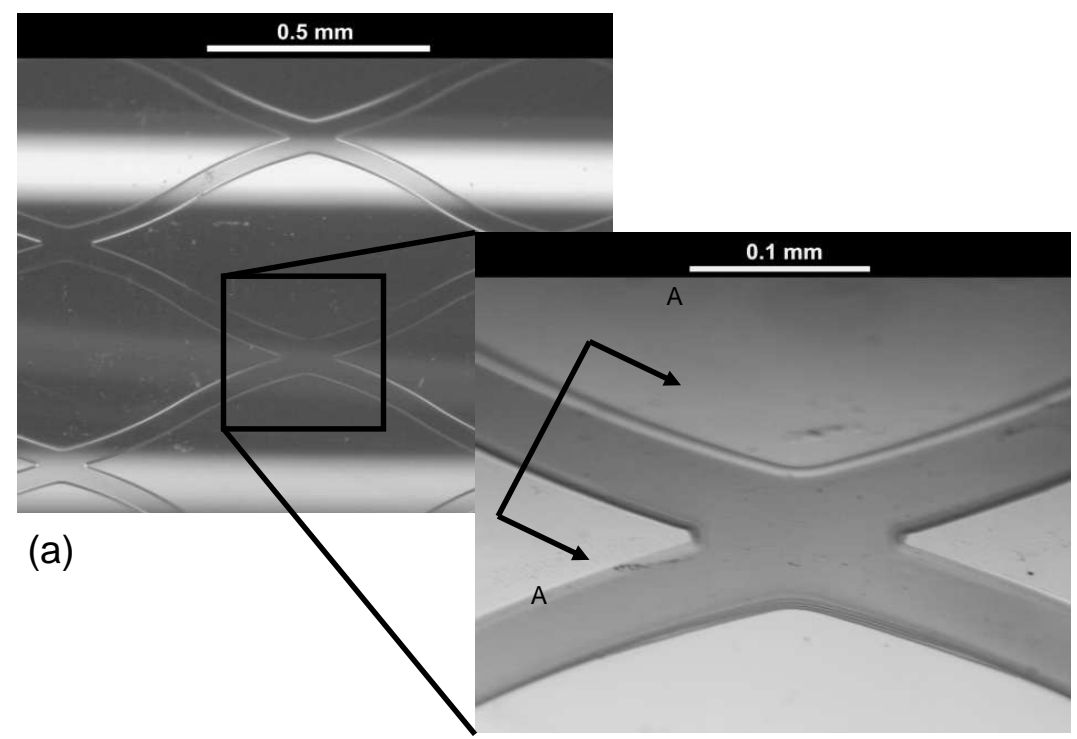

\section{Photoresist geometry Isotropic theory Optimized solution \\ Cross section A-A Cross section A-A Cross section A-A}

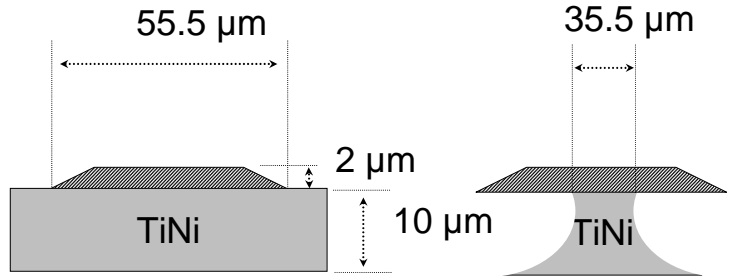

(b) (c)

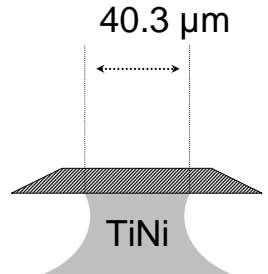

(d)

Figure 4.51: Schematic comparison of the isotropic theory and our optimized TiNi etchant solution. (a) Optical micrograph from a photoresist structured with a stent like structure $55 \mu \mathrm{m}$ feature size, (b) A-A system cross section, (c) Isotropic etching - final feature size $35.5 \mu \mathrm{m}$, (d) Optimized solution - final feature size $40.3 \mu \mathrm{m}$.

was developed to rotate the cylindrical substrate, moving the etchant to the specimen surface and moving away the reaction products, reducing the cited effects. Figure 4.52 shows a schematic drawing of the etching rotational device (a) electric motor, (b) Teflon recipient and (c) sample holder.

This etching rotational device solved the etching inhomogeneity effect, as shown in 


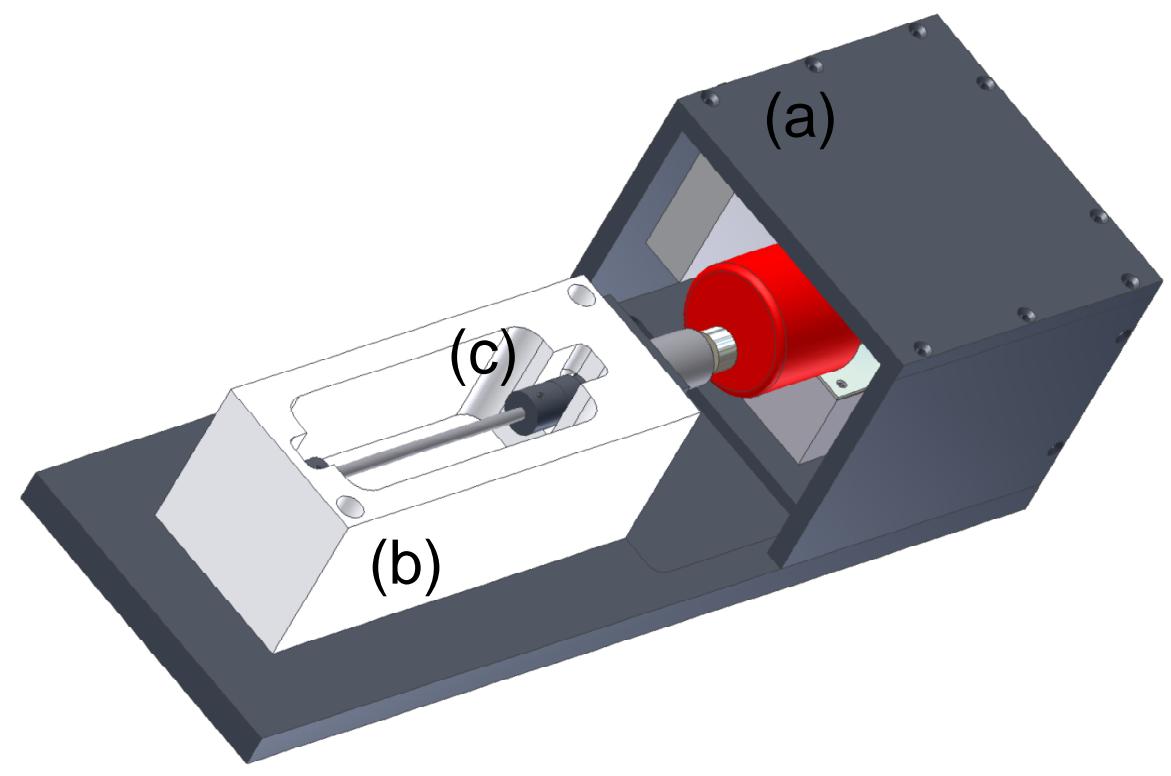

Figure 4.52: Etching rotational device (a) electric motor, (b) Teflon recipient and (c) sample holder.

figure 4.53. Figure 4.53 (a) shows $1.5 \mathrm{~mm}$ diameter TiNi cylindrical thin film which was static wet etched before sacrificial layer etching and figure 4.53 (b) shows $1.5 \mathrm{~mm}$ diameter TiNi cylindrical thin film which was dynamic wet etched before sacrificial layer etching.

The etching inhomogeneity was also observed when the thickness along the sample is not homogenous. It was shown in section 4.14, that the use of a shutter during sputtering increases the film thickness homogeneity, thereby decreasing the etching inhomogeneity effect.

Using the same sputtering, photo lithography and wet etching a crystalline TiNi cylindrical thin film and an amorphous one were wet etched in order to compare the dependency of lateral ratio within the film morphology. Figure 4.54 shows a SEM picture of a structured (55 $\mu \mathrm{m}$ feature size) TiNi cylindrical crystalline thin film wet etched. The lateral ratio is very high, approximately 2.84, and the final feature size decrease from 55 to $26 \mu \mathrm{m}$. 

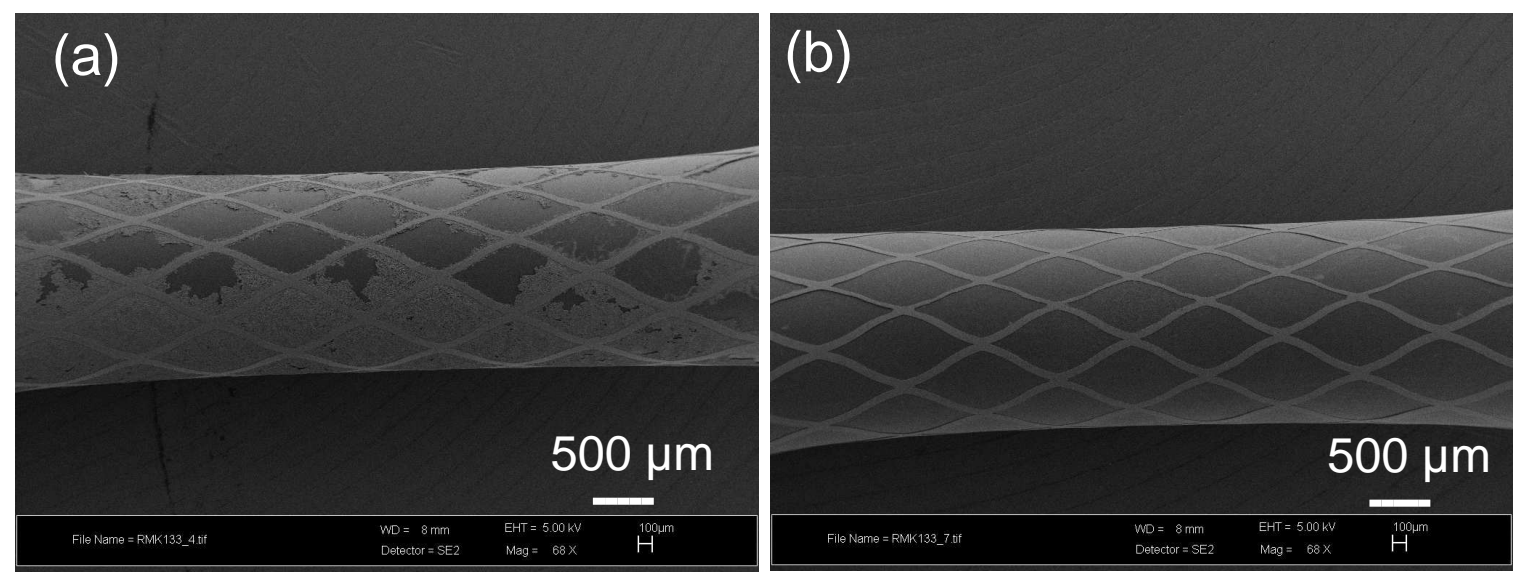

Figure 4.53: (a) shows $1.5 \mathrm{~mm}$ diameter TiNi cylindrical thin film which was static structured before sacrificial layer etching and figure (b) shows $1.5 \mathrm{~mm}$ diameter TiNi cylindrical thin film which was dynamic structured before sacrificial layer etching.

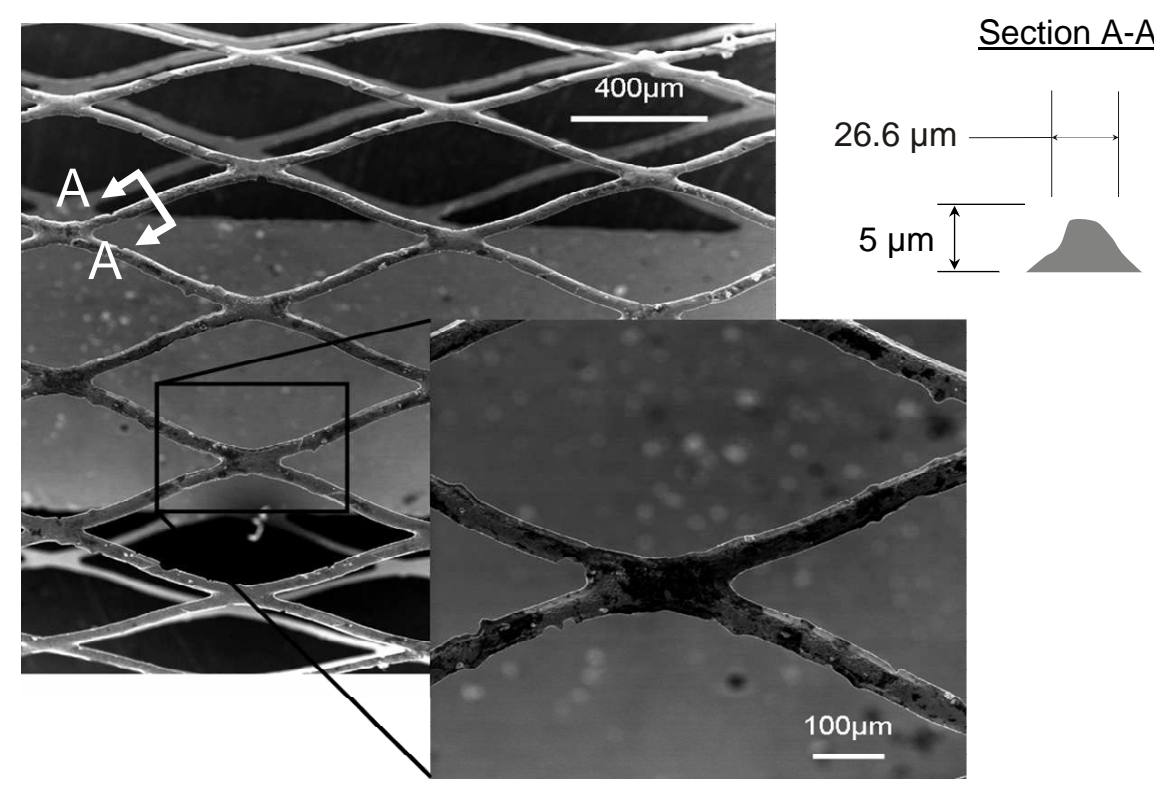

Figure 4.54: Wet etched crystalline TiNi cylindrical thin film, $5 \mu \mathrm{m}$ thickness.

Figure 4.55 shows a SEM picture of a structured ( $55 \mu \mathrm{m}$ feature size) TiNi cylindrical amorphous thin film wet etched. The film did not show a significant undercutting as it 
was found in crystalline samples.

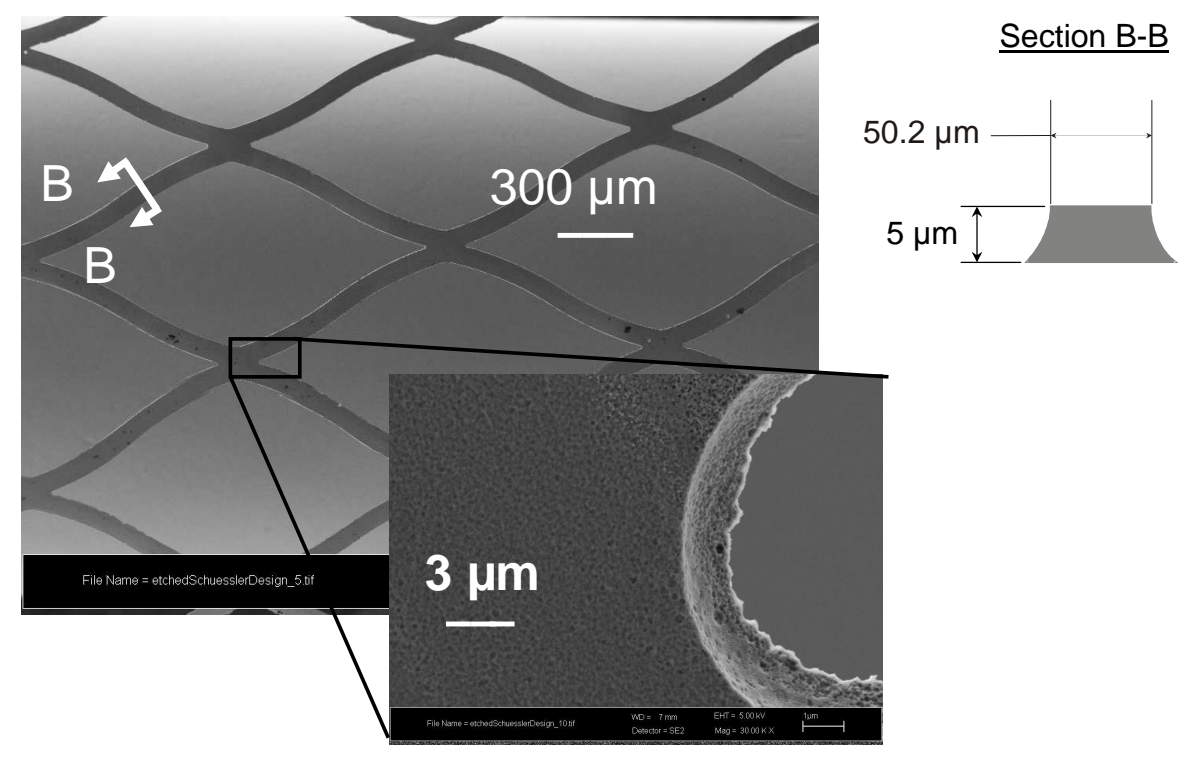

Figure 4.55: Wet etched amorphous TiNi cylindrical thin film, $5 \mu \mathrm{m}$ thickness.

There are two explanations for this behavior: first the binding energy of $\mathrm{Ti}$ and $\mathrm{Ni}$ in an amorphous state is lower than in a crystalline state, thus it is easier to etch TiNi film in amorphous state. Second, the grain boundary etching, which on crystalline TiNi will occur first allowing the uncontrolled etching. Many devices, with different diameters and thickness were produced in the course of this research project.

Figure 4.56 shows a $1.5 \mathrm{~mm}$ diameter structured TiNi thin film stent like device fabricated by means of magnetron, cylindrical lithography and wet etching. 
4.3 Structuring cylindrical TiNi thin films
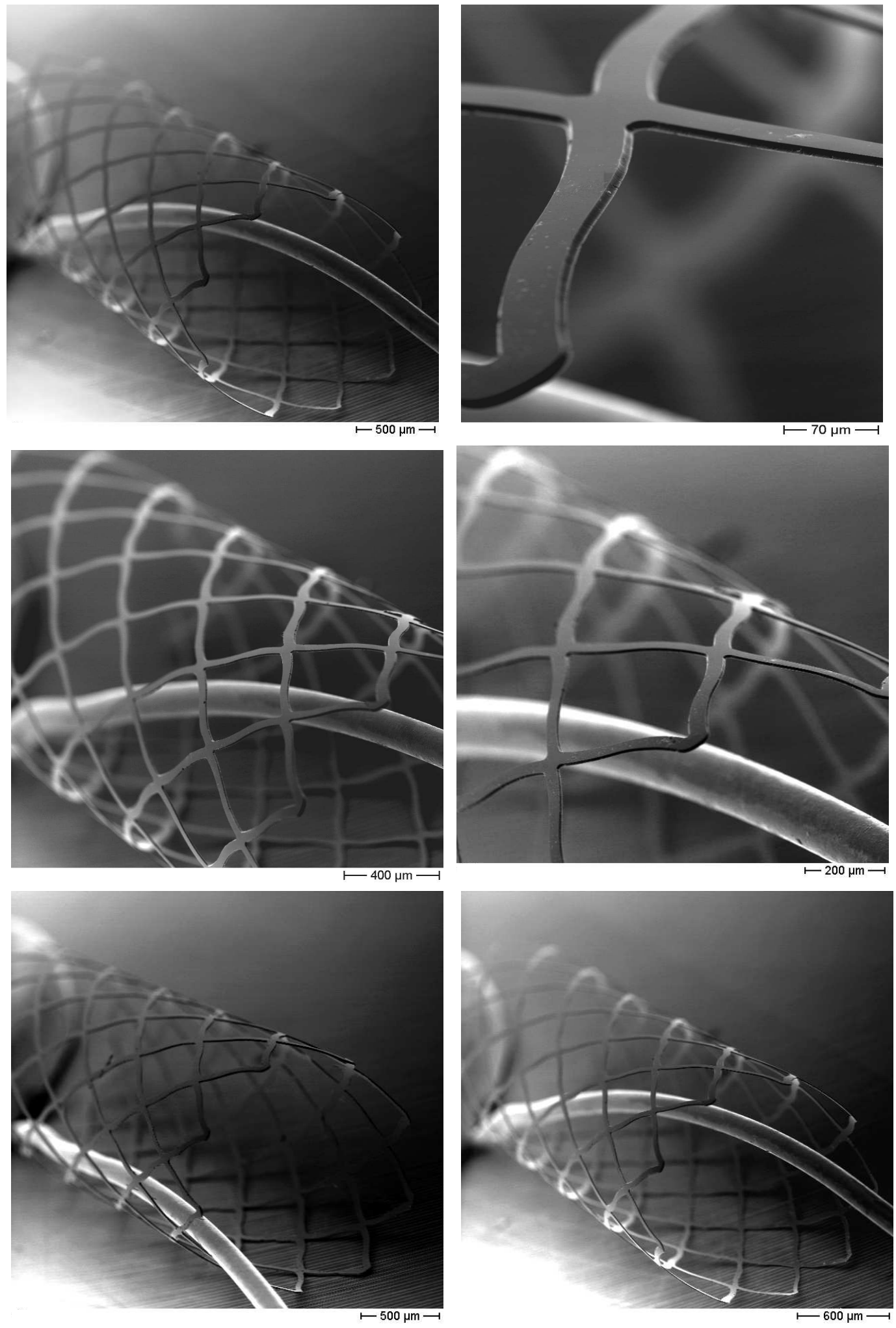

Figure 4.56: $1.5 \mathrm{~mm}$ diameter structured TiNi thin film stent like device by means of magnetron, cylindrical lithography and wet etching. A Cu capillary was used to hold the sample in the SEM sample holder. 
SEM figure 4.57 shows a $3.5 \mathrm{~mm}$ diameter structured TiNi thin film stent like device. The thickness of this device was $10 \mu \mathrm{m}$ and its length $30 \mathrm{~mm}$.

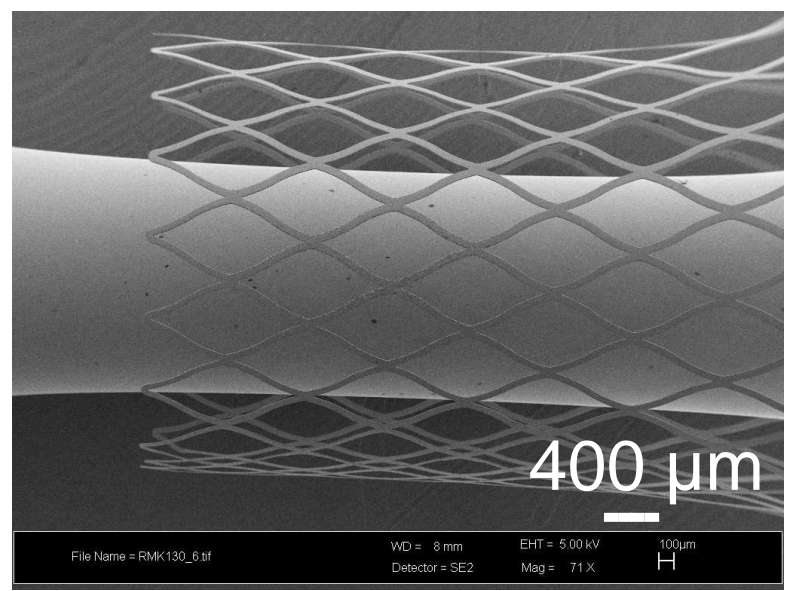

Figure 4.57: $3.5 \mathrm{~mm}$ diameter structured TiNi thin film stent like device by means of magnetron sputtering, cylindrical lithography and wet etching process. A Cu capillary was used to hold the sample in the SEM sample holder.

As described in section 2.5, crimping is the last production step of a TiNi stent production process flow and consists of outer diameter reduction through applied radial compression. Thus, a crimping simulation was performed in a $1.5 \mathrm{~mm}$ diameter TiNi cylindrical structured thin film device, using a glass cone with final diameter $0.6 \mathrm{~mm}$. Crimping simulation test principle is illustrated in figure 4.58 .

\section{$1.5 \mathrm{~mm}$ diameter structured TiNi thin film}

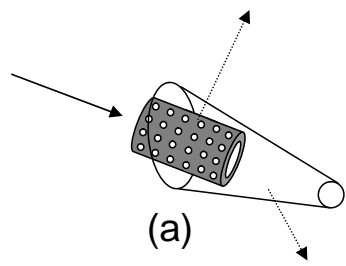

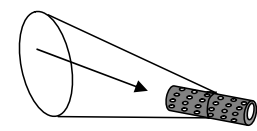

(c)

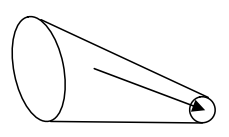

(d)

\section{Glass cone}

final diameter $=0.6 \mathrm{~mm}$

Figure 4.58: Crimping simulation test principle. The sequence (a), (c) and (d) corresponds to the optical micrograph test figure 4.59 . 
This simulation test was carried out to confirm the mechanical properties of the cylindrical structured TiNi thin films. The generic stent design (see figure 4.45) was used to structure the tested cylindrical TiNi thin film. It is well known that the crimping process depends on the material property as well as the device design [88]. Thus, this simulation was conduced to test the self expansion material property. Figure 4.59 shows optical micrographs from the crimping simulation, (a) TiNi thin film stent was introduced in a $0.6 \mathrm{~mm}$ final diameter glass filler, (b) and (c) device was moved in using a pincer, (d) device returns to its original shape after passing trough the filler aperture.
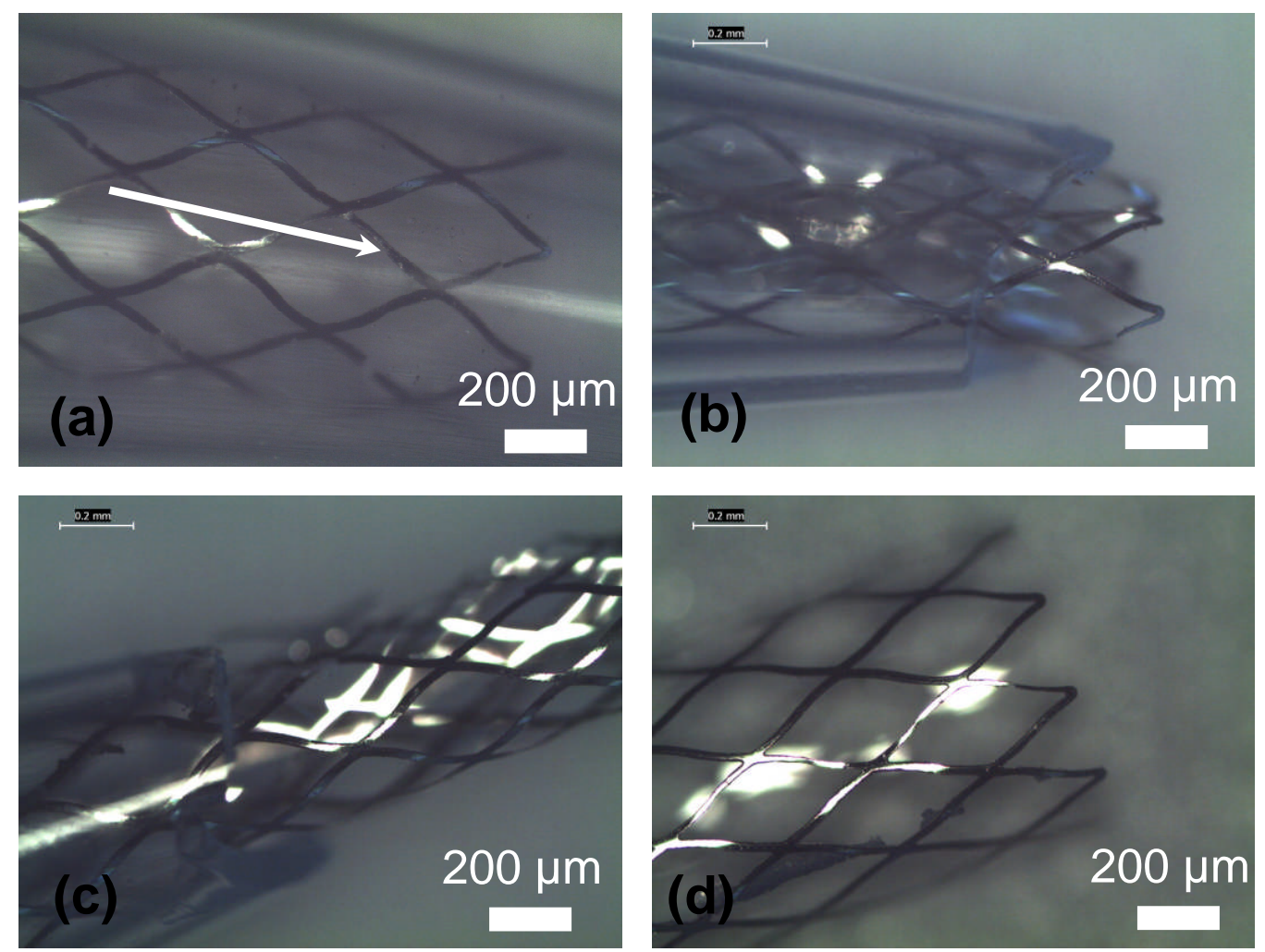

Figure 4.59: Optical micrographs from the crimping simulation realized on a $1.5 \mathrm{~mm}$ diameter TiNi thin film stent device, (a) TiNi thin film stent was introduced in a $0.6 \mathrm{~mm}$ final diameter glass filler, (b) and (c) device was moved in using a pincer, (d) device returns to its original shape after passing trough the filler aperture.

The crimping simulation test, together with stress-strain and hoop force tests (see 
section 4.2.3 confirm the exceptional mechanical properties of cylindrical TiNi thin films fabricated by magnetron sputtering, cylindrical lithography and wet etching.

\subsubsection{Method used to fabricate fine structured TiNi "thick" films by magnetron sputtering}

A design limitation due to wet etching isotropic behavior was also observed when the feature mesh is smaller than $20 \mu \mathrm{m}$ and the film thicker than $10 \mu \mathrm{m}$. In order to address this issue, a method to fabricate "thick" films with very small mesh was developed and registered in May 2009 as a patent, based on UV lithography and wet etching technology. This method was sucessfuly applied to planar films. Figure 4.60 shows a schematic flow of the developed method.

(a)

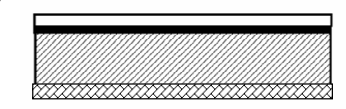

(b)

(c)
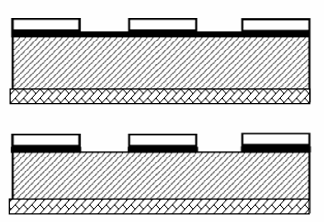

(d)

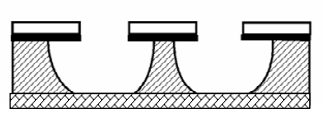

(e)

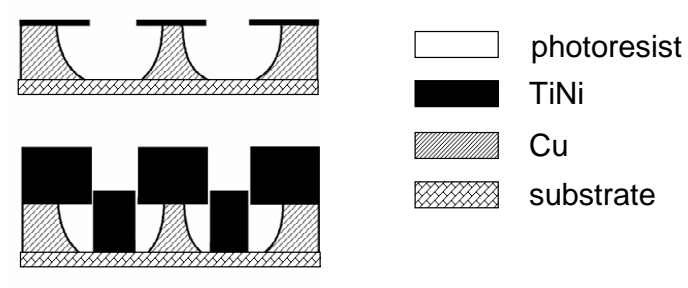

(g)

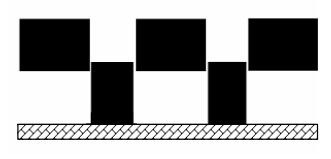

Figure 4.60: Method flow, (a) after sputtering of Cu sacrificial layer and NiTi thin film photoresist is spin coated (b) patterning of photoresist by means of UV lithography (c) TiNi thin film wet etch (d) sacrificial layer wet etching (e) removing photoresist (f) deposition thick TiNi film (g) sacrificial layer wet etch.

The first step of this method is the deposition of a $\mathrm{Cu}$ sacrificial thin film layer, figure 4.60 (a). After the sacrificial layer deposition, TiNi thin film with $1.5 \mu \mathrm{m}$ is deposited, figure 4.60 (b). Lithography is used to structure the film. The $1.5 \mu \mathrm{m}$ TiNi thin film was then wet etched with an HF solution, figure 4.60 (c). The sacrificial layer is then wet etched using a standard BASF Selectipur Chromium Etch, an ammonium cerium 
(IV) nitrate based etchant, which does not etch TiNi thin film, figure 4.60 (d). A "mushroom"-like structure is formed, due to the isotropic behavior of the wet etching process. This structure allows the growth of the film on top of the pre-structured mesh, avoiding connection between the films. The photoresist is removed, figure 4.60 (e), and the TiNi "thick" film is deposited using the same parameters as before, figure 4.60 (f). To get the film free from the substrate the sacrificial layer is then completely wet etched, figure 4.60 (g). Figure 4.61 shows a TiNi structured thin film of $1.5 \mu \mathrm{m}$ thickness, after sacrificial layer wet etching. This step corresponds to the schematic step 4.61 (e). The "mushroom"-like structure can be observed.

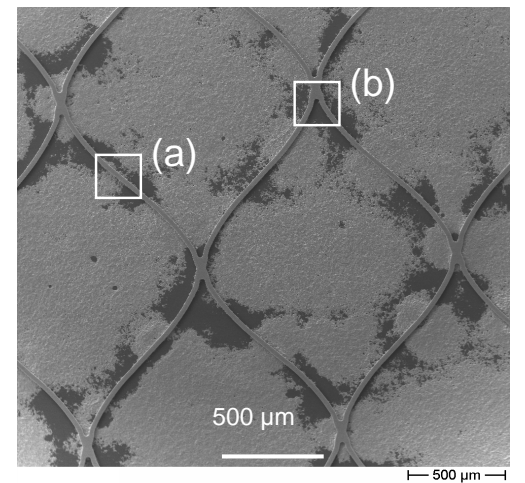

Cross Section

$$
\text { A-A }
$$

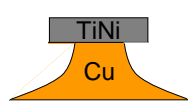

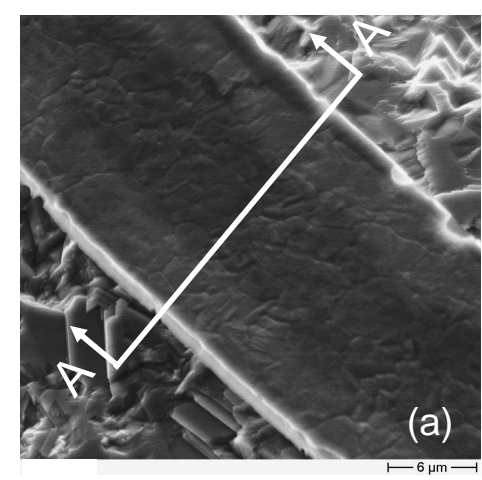

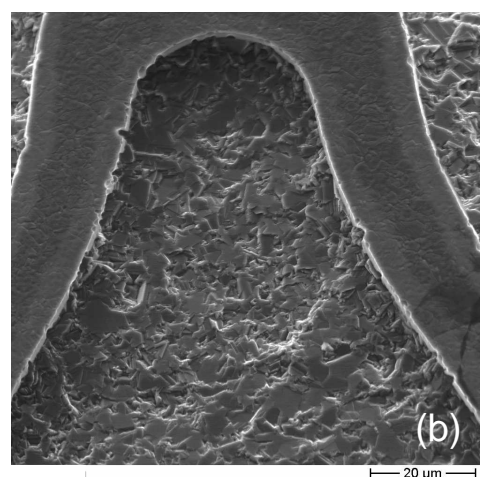

Figure 4.61: TiNi structured thin film of $1.5 \mu \mathrm{m}$ thickness, after sacrificial layer wet etching and removal of the photoresist.

Figure 4.62 shows a Scanning Electron Microscope (SEM) image after the deposition of $25 \mu \mathrm{m}$ TiNi film. A channel is formed between the film on top of the patterned structure.

The final step is to get the film free from the substrate which means the wet etching 


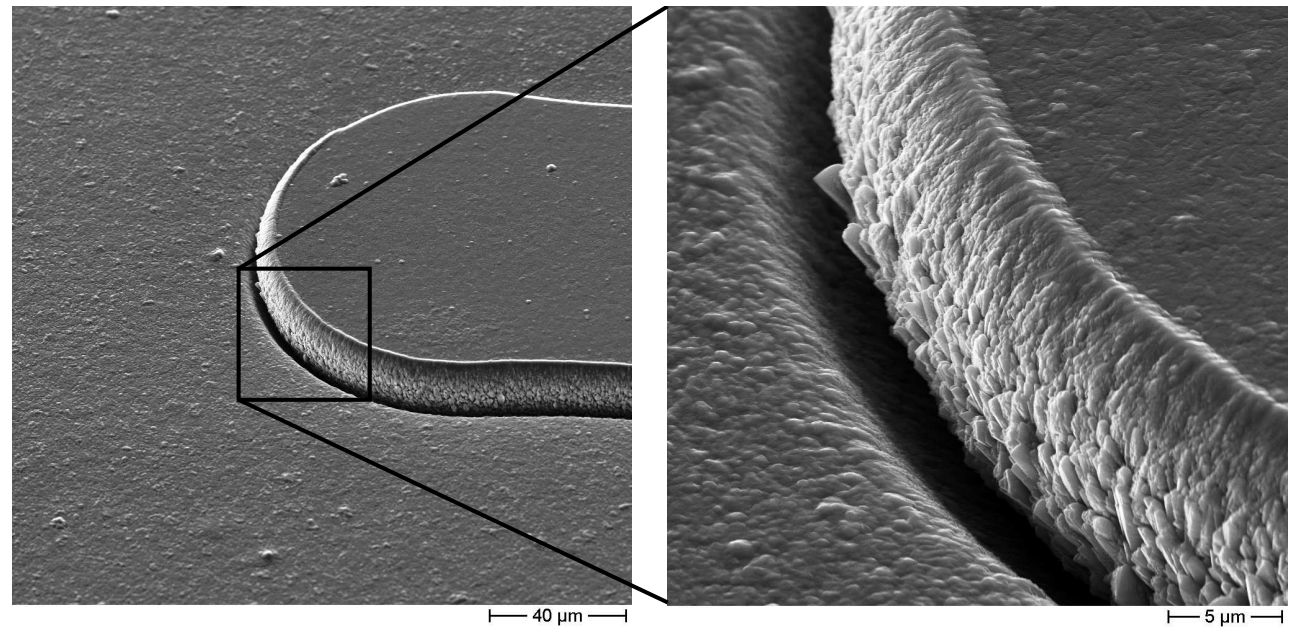

Figure 4.62: Example of the method step (f) after deposition of $25 \mu \mathrm{m}$ TiNi film.

of the sacrificial layer. The etchant will then be able to etch the sacrificial layer due to the channel created between the film on top of the patterned structure and the bottom. Figure 4.63 shows a SEM image of a $24 \mu \mathrm{m}$ thick TiNi film with a mesh width of $25 \mu \mathrm{m}$.

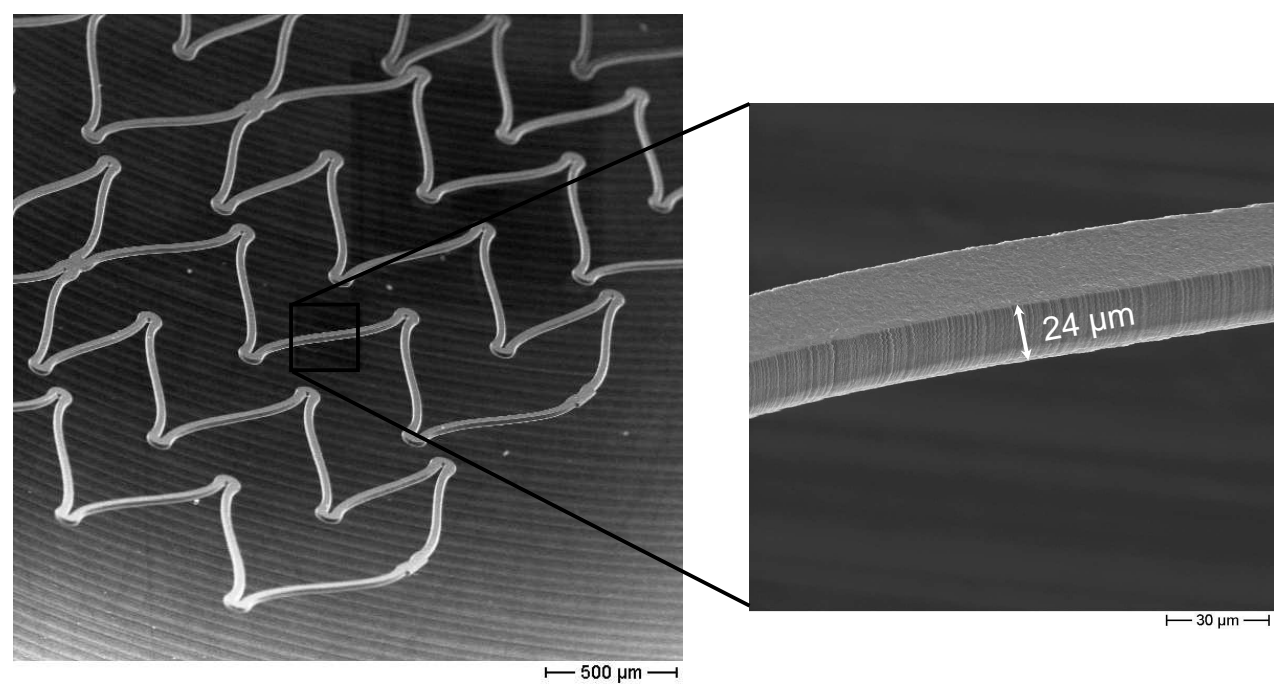

Figure 4.63: Example of the method step (g) after sacrificial layer wet etched.

This method is a promising substitute for the solid state laser technology, as the aspect ratio of this method is comparable to the 10:1 ratio achieved by laser cut technology. In 
addition, it does not produce small burrs, as well as a heat affected zone or micro cracks. This method further avoids a possible contamination of the TiNi material with carbides. Figure 4.64 shows that using this method it is possible to achieve film thickness up to $50 \mu \mathrm{m}$.

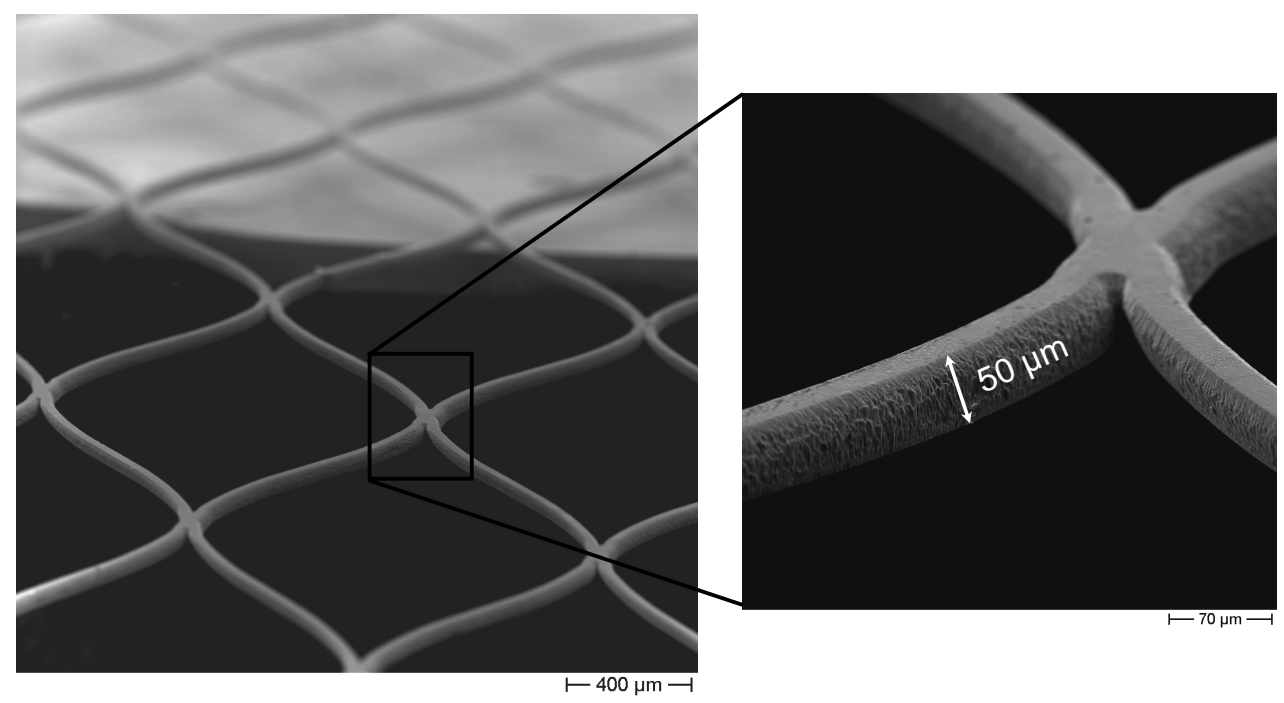

Figure 4.64: TiNi structured thin film with $50 \mu \mathrm{m}$ thickness and $20 \mu \mathrm{m}$ mesh. 


\section{Chapter 5}

\section{Summary}

This dissertation described for the first time the fabrication and characterization of structured cylindrical TiNi thin films by magnetron sputtering, lithography and wet etching based on previous developments of superelastic shape memory thin films. The TiNi thin films devices fabricated in this work have high potential for application as vascular implants, e.g. stents.

The deposition of TiNi thin films on a cylindrical substrate employs a specific device which allows an in situ rotation of the substrate during the sputtering process and the use of a slit aperture to control the deposition angle. Axial and radial directions of the film were tested under tensile load. Both film directions showed comparable mechanical properties to TiNi planar films. Superelastic plateau, closed loop hysteresis and a strain up to $6 \%$ were observed.

The crystallization mechanisms as a function of film thickness, annealing time and substrate were analyzed in cylindrical deposited TiNi thin film. XRD and TEM analysis have shown that 5 minutes annealing time at $650{ }^{\circ} \mathrm{C}$ first step and $450{ }^{\circ} \mathrm{C}$ second step is enough to crystallize the cylindrical TiNi thin films, which is essential for the fabrication of hybrid devices (e.g. combination of thin film stents and conventional TiNi devices). Figure 5.1 shows a hybrid device developed with the use of the methods developed during this dissertation [91].

A rotational UV lithography device based on the standard photolithography pro- 


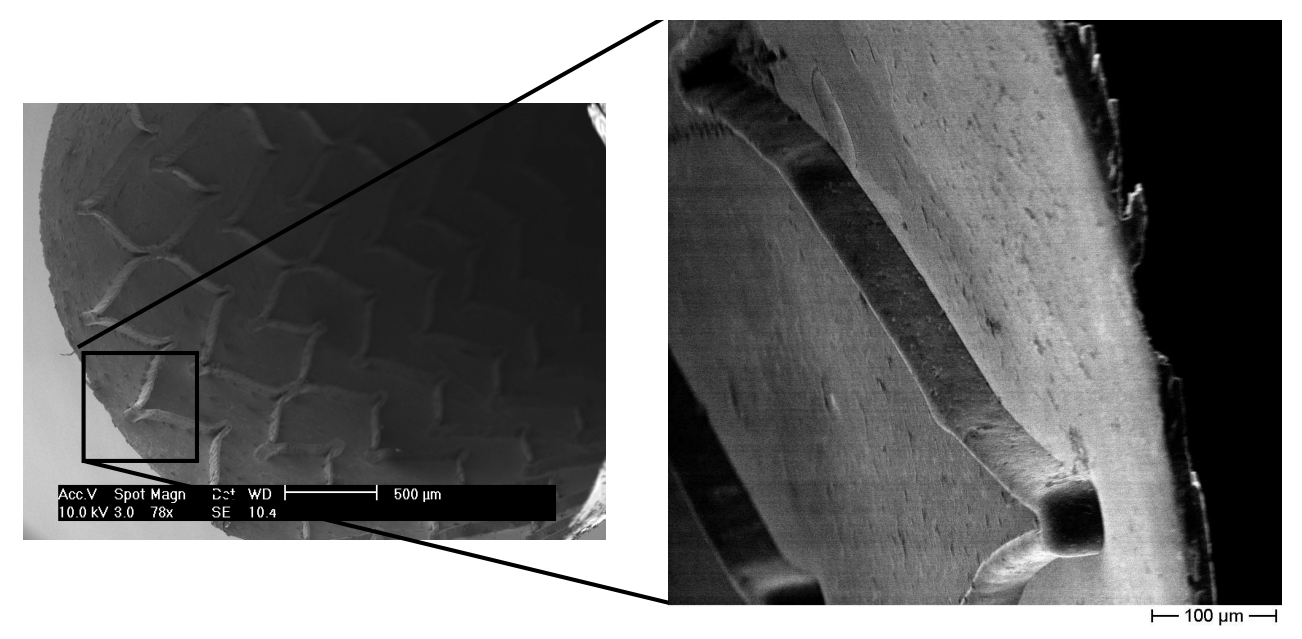

Figure 5.1: Hybrid device fabricated with the use of the methods developed during this dissertation.

cess was developed and characterized for patterning of cylindrical substrates of different diameters and lengths with a feature resolution down to $5 \mu \mathrm{m}$ and an axial resolution $(\Delta y)$ of $3 \mu \mathrm{m}$. Wet etching technology was used for the pattern transfer to the cylindrical TiNi films. The influence of etching parameters: movement of the etchant and etchant composition on the etching rate and lateral ratio (relation between horizontal etching rate $\left(\dot{E}_{h}\right)$ and the vertical etching rate $\left.\left(\dot{E}_{v}\right)\right)$ was also analyzed and optimized during this research. Thus, this wet etching analysis allowed us to optimize the etchant solution thereby achieving a lateral ratio of 0.76 .

A deposition method was developed and patented to fabricate "thick" films with a very small mesh, based on UV lithography and wet etching technology. This method is a promising substitute for the solid state laser technology, as the aspect ratio of this method is comparable to the 10:1 ratio achieved by laser cut technology. In addition, it does not produce small burrs, as well as a heat affected zone or micro cracks. This method further avoids a possible contamination of the TiNi material with carbides.

A medicine device validation on e.g. stents, requires not only the mechanical feasibility but also several in vivo experiments. The group of Prof. Dr. med. Martin Heller, University Medical Center Schleswig Holstein, have started the first in vivo experiments, using TiNi thin films devices fabricated during this work. A radiopacity test was realized 
to assure the visibility of the thin film device after introduction. Figure 5.2 shows first results obtained with the use of an ultrasound equipment, which confirms the radiopacity of a $10 \mu m$ cylindrical TiNi thin film. Further experiments e.g. rats experiments, etc. are scheduled to be realized in the near future.
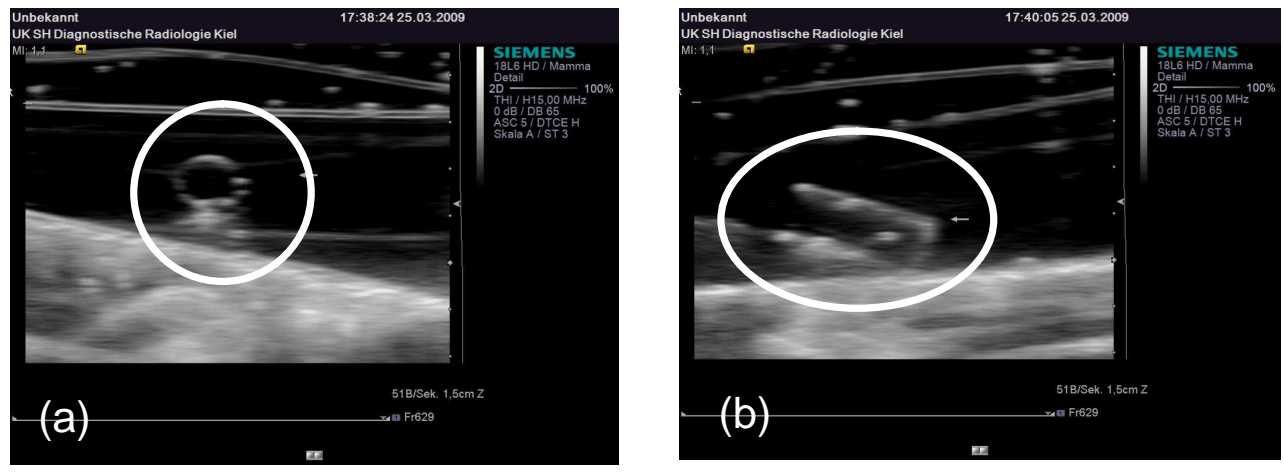

Figure 5.2: Radiopacity experiment using a ultrasound equipment, (a) frontal view, (b) lateral view.

As described in this dissertation, the deposition rate achieved on cylindrical deposition is reduced by a factor of 15 due to the slit aperture introduced to control the deposition angle. Using thin film deposition equipment dedicated for planar substrates this was essential to achieve the required mechanical and superelastic properties of the cylindrical TiNi thin films. The resulting maximum film deposition of approximately $1 \mu \mathrm{m} / \mathrm{h}$ limits the achievable wall thickness in practice to values bellow $15 \mu \mathrm{m}$. Furthermore, this approach is very cost-effective both in terms of labor and target costs. Thus, in view of possible commercialization of these new devices the development of a new deposition equipment using e.g. hollow cathode or multiple target arrangement, resulting in much higher deposition rates, is the essential next step, have to be developed and characterized in order to fabricate cylindrical films with higher wall thickness. 


\section{Chapter 6}

\section{Acknowledgments}

After three years of work, it is amazing how many people I have to thank! I would like to thank my "Doktorvater" Prof. Dr. Eckhard Quandt, who gave me the opportunity to join his group. In addition, I would like to thank my supervisor Dr. "general" Christiane Zamponi, who guided me during this three years. I will never forget our nice discussions and of course our "fights".

I want to thank the whole Institute for Inorganic Functional Materials for the excellent friendly atmosphere, support and help. Especially, Dipl.-Ing. Thomas Metzing for the engineering advices as well as the help with "my baby" (magnetron sputtering device); Gislinde Schröder for the help with the German bureaucracy; Dipl. Ing. Andre Piorra for rich conversations and Dipl.-Wi.-Ing. Christoph Bechtold (the "LEGOman") for the friendship and also for the technical help, especially with programming.

I would like to thank Prof. Franz Faupel, who has motivated me to continue in the academic world after my master studies and his Institute. Especially, PD Dr. Klaus Rätzke for the nice discussions about thin films and Dipl.-Ing. Stephan Rehders for the always helpful engineering advices.

Special thanks go to the best University Mechanical Shop ever, the TF Uni Kiel Mechanical Shop. Meister Bernt Neumann and the whole mechanical shop team know how to work fast and with quality!

I am also grateful to my previous extinct Institute, Smart Materials, at Caesar (Cen- 
ter of Advanced European Studies and Research - Bonn - Germany) for providing the necessary support to start my research. Especially, Dipl.-Ing. Michael Koch, who has supported the design of at least two devices presented in this dissertation and Angelika Sehrbrock for the TEM analysis.

I further want to thank Fiona Malkin for final proof-reading and the Kieler friends for the wonderful time.

I would like to thank the one who is the main motivation to be here in Germany and conclude this dissertation, my wife Katharina. The love and support with which she has provided me during these years I will never forget. In addition, owe gratitude to my entire German family (Benhard, Angela, Franzi und Jonas) for supporting me and receiving me as part of the family.

Finally, I would like to thank my family, (Márcia, Ângelo, Marcello e Ana Paula) for their support especially since my decision to come to Germany. The love that they have provided has allowed me to pursue my dreams. Without them I could not finish this work. 


\section{Chapter 7}

\section{Own publications and patent applications}

The publications and patent applications listed below were written during this dissertation period.

\section{Publications}

1. R. Lima de Miranda, C. Zamponi, E. Quandt, Rotational UV Lithography Device for Cylindrical Substrate Exposure, Review of Scientific Instruments, 2009, 015103

2. R. Lima de Miranda, C. Zamponi, E. Quandt, Fabrication of TiNi Thin Film Stents, Smart Materials and Structure, 2009 - In Print

3. C. Zamponi, R. Lima de Miranda; M. Carey (ed.), Thin Film Shape Memory Alloys, Chapter 15, Cambridge University Press, 2009 - In Print

4. F. Kahleyss, R. Lima de Miranda, T. Surmann, C. Zamponi, D. Biermann, E. Quandt, Processing and Damping properties of sputtered NiTi Thin Films for Tools in Machining Process, International Journal of Machine Tools and Manufacture, 2009 - submitted 


\section{Patent applications}

1. Stent mit dünnen Stegen - M/CAN-072-DE (in approval)

2. Methode zur Realisierung eines Implantats mit kontrollierter Bioabsorption - M/CAN060-DE (in approval) 


\section{Bibliography}

[1] A. Schuessler and M. Piper. Boundaries for the use of nitinol in medial application. Proceedings of the International Conference on Shape Memory and Superelastic Technologies, pages 563-571, 2004.

[2] G. Mani, M. D. Feldman, D. Patel, and C. M. Agrawal. Coronary stents: A materials perspective. Biomaterials, 28(9):1689-1710, Mar 2007.

[3] R. A. Schatz, J. C. Palmaz, F. O. Tio, F. Garcia, O. Garcia, and S. R. Reuter. Balloon-expandable intracoronary stents in the adult dog. Circulation, 76, No. $2,: 450-457,1987$.

[4] K Otsuka and C.M. Wayman, editors. Shape Memory Materials. Cambridge University, 1998.

[5] C. Zamponi, H. Rumpf, B. Wehner, J. Frenzel, and E. Quandt. Superelasticity of free-standing niti films depending on the oxygen impurity of the used targets. Materialwissenschaft und Werkstofftechnik, 35:359-364, 2004.

[6] N. Shevchenko, M.-T. Pham, and M. F. Maitz. Studies of surface modified niti alloy. Applied Surface Science, 2004.

[7] H. Rumpf, C. Zamponi, C. Bourauel, and E. Quandt. Fabrication of superelastic niti tubes by magnetron sputtering. In SMST, pages 211-216, 2004.

[8] H. Rumpf, T. Walther, C. Zamponi, and E. Quandt. High ultimate tensile stress in nano-grained superelastic niti thin films. Materials Science and Engineering, A 415:304-308, 2006. 
[9] A. Schuessler. Laser processing of nitinol materials. SMST-2000 Conference Proceedings, 2000.

[10] M. S. Wechsler, D. S. Lieberman, and T. A. Read. On the theory of the formation of martensite. Transactions AIME, pages 1503-1508, 1953.

[11] J. S. Bowles and J. K. Mackenzie. The crystallography of martensite transformation i. ACTA Metallurgica, 2:129-137, 1954.

[12] J. S. Bowles and J. K. Mackenzie. The crystallography of martensite transformation ii. ACTA Metallurgica, 2:138-147, 1954.

[13] G. V. Kurdjumov and L. G. Khandros. Dokl. Nauk SSSR, 211:66, 1949.

[14] Y. I. Paskal and L. A. Monasevieh. Phenomenological characteristics of martensitic hysteresis. Russian Physics Journal, 21:1466-1471, 1978.

[15] T. W. Duerig and R. Zadno. An engineers perspective of pseudoelasticity. Butterworth-Heinemann, London, 1990.

[16] G. Guenin. Martensitic transformation: Phenomenology and the origin of the twoway memory effect. Phase Transitions, 14:165-175, 1988.

[17] G.S Firstov, J. Van Humbeeck, and YU. N Koval. High temperature shape memory alloys problems and prospects. Journal of Intelligent Material Systems and Structures, 17:1041-1047, 2006.

[18] W. J. Buehler and J. W. Gilfrich. Role of oxygen in reducing silicon contamination. Wiley RC. J Appl Phys, 34:1473., 1963.

[19] K. Otsuka and X. Ren. Physical metallurgy of ti-ni-based shape memory alloys. Progress in Materials Science, 50:511-678, 2005.

[20] H. Xu and I. Moller. Effect of mechanical vibration, heat treatment and ternary addition on the hysteresis in shape memory alloys. Journal of Materials Science, 26:1473-1477, 1991. 
[21] T. B. Massalski, H. Okamoto, P. R. Subramanian, and L. Kacprzak. Binary alloy phase diagrams. Materials Park, OH: ASM International, 2:2874, 1990.

[22] M. Nishida, C. M. Wayman, and T. Honma. Precipitation processes in nearequiatomic tini shape memory alloys. Metall. Trans. A, 17A:1505, 1986.

[23] W. Tang. Thermodynamic study of the low-temperature phase b19' and the martensitic transformation in near-equiatomic ti-ni shape memory alloys. Metallurgical and materials transactions. A, 28:537-544, 1997,.

[24] Memory-Metalle GmbH. Selected properties of niti-based alloys. 2000.

[25] G.K Wehner and G.S. Anderson. Handbook of Thin Film Technology. 1970.

[26] B. Chapman. Glow Discharge Processes. Willey, New York, 1980.

[27] S. A. Campbell. The Science and Engineering of Microelectronic Fabrication. Oxford University Press, 2001.

[28] M. L. Tarng and G. K. Wehner. Alloy sputtering studies with in situ auger electron spectroscopy. Journal of Applied Physics, 42(6):2449-2452, 1971.

[29] I. Neshev, V. Hamishkeev, V. S. Chernysh, S. Postnikov, and B. Mamaev. Angular distributions of sputtered particles from niti alloy. Pergamon Press Ltd, 1993.

[30] H.H. Andersen, V. Chernysh, B. Stenum, T. Sørensen, and H.J. Whitlow. Measurements of angular distributions of sputtered material as a new tool for surfacesegregation studies: Segregation in cupt alloys. Surface Science, 123(1):39 - 46, 1982.

[31] Shingo Ichimura, Hazime Shimizu, Hiroshi Murakami, and Ishida Yoichi. Effect of surface segregation on angular distributions of atoms sputtered from binary alloys. Journal of Nuclear Materials, 128-129:601 - 604, 1984. 
[32] A. A. Soloviev, N. S. Sochugov, K. V. Oskomov, and N. F. Kovsharov. Film thickness distribution in magnetron sputtering system with the round cathode. In International Congress on Radiation Physics, High Current Electronics and Modification of Materials, 2006.

[33] J. A. Thornton. High rate thick film growth. Ann. Rev. Mater. Sci., 7:239-260, 1977.

[34] M. Ohring. Materials Science of Thin Films. Academic Press, 2002.

[35] John A. Thornton. The microstructure of sputter-deposited coatings. Journal of Vacuum Science \& Technology A: Vacuum, Surfaces, and Films, 4(6):3059-3065, 1986.

[36] D. S. Grummon, J. Zhang, and T. J. Pence. Relaxation and recovery of extrinsic stress in sputtered titanium-nickel thin films on (100) si. Materials Science and Engineering A, 273:722-726, 1999.

[37] M. J. Madou. Fundamentals of Microfabrication - The Science of Miniturization. CRC Press, 2002.

[38] L. D. Landau and B. Levich. Dragging of a liquid by a moving plate. Acta Physicochim. USRR, 17:42-54, 1942.

[39] G. Geikas and B. Ables. Kodak Photoresist Seminar, page 22, 1968.

[40] R. C MacLaurin. Light. Columbia University Press, 1909.

[41] O. Nalamasu, M. Cheng, A. G. Timko, V. Pol, E. Reichmanis, and L. F. Thompson. An overview of resist processing for deep-uv lithography. Journal of Photopolymer Science and Technology, 4:299-318, 1991.

[42] O. Nalamasu, F. M. Houlihan, E. Reichmanis, R. Cirelli, and A. Timko. An overview of the role of materials technology for $193 \mathrm{~nm}$ lithography. Polymer Material Science Engineering, 84:207, 2001. 
[43] M. Köhler. Etching in Microsystem Technology. Wiley-VCH, 1999.

[44] J. Ryhänen. Biocompatibility Evaluation of Nickel-Titanium Shape Memory Metal Alloy. PhD thesis, University of Oulu,Departments of Surgery, Anatomy and Pathology, 1999.

[45] M. Dolce and D. Cardone. Fatigue resistance of sma-martensite bars subjected to flexural bending. International Journal of Mechanical Sciences, 2003.

[46] S. Miyazaki, K. Mizukoshi, T. Ueki, T. Sakuma, and Yinong Liu. Fatigue life of ti-50 at.alloy wires. Materials Science and Engineering A, 273-275:658 - 663, 1999.

[47] R.L. Holtz, K. Sadananda, and M.A. Imam. Fatigue thresholds of ni-ti alloy near the shape memory transition temperature. International Journal of Fatigue, 21:137$145,1999$.

[48] K.N Melton and O Mercier. Fatigue of niti thermoelastic martensites. Acta Metallurgica, 27(1):137 - 144, 1979.

[49] P. Poncet, P.H. Adler, S. Carpenter, and M.H. Wu. Manufacture of nitinol tubing. In ASM Materials \& Processes for Medical Devices Conference, Anaheim, 2003.

[50] J. MacWilliams. Optimization of nitinol shape setting through post laser cutting processing. Medical Device Materials III: Proceedings from the Materials \&5 Processes for Medical Devices Conference 2005, pages 69-72, 2006.

[51] P. P. Poncet. Applications of superelastic nitinol tubing. Technical report, MEMRY Corporation 4065 Campbell Avenue - Menlo Park CA 94025 - USA, 2000.

[52] P. R. Subramanian, S. Krishnamurthy, S. T. Keller, and M. G. Mendiratta. Processing of continuously reinforced ti-alloy metal matrix composites (mmc) by magnetron sputtering. Materials Science and Engineering A, 244(1):1 - 10, 1998.

[53] I. Zhitomirsky and L. Gal-Or. Formation of hollow fibers by electrophoretic deposition. Materials Letters, 38(1):10 - 17, 1999. 
[54] S. Shingubara, H. Kotani, H. Sakaue, F. Nishiyama, and T. Takahagi. Correlation between agglomeration of a thin film and reflow filling in a contact hole for sputtered al films. Journal of Vacuum Science \&s Technology B: Microelectronics and Nanometer Structures, 17(6):2553-2558, 1999.

[55] K. Zhao, B. C. Zhang, L. F. Wang, Z. L. Shen, D. Q. Lu, and D. L. Xie. A cylindrical magnetron sputtering system for depositing metal-aluminium nitride cermet solar coatings onto batches of tubes. J. Vac. Sci. Technol. A, 16:628-362, 1998.

[56] H. G. Prengel, P. C. Jindal, K. H. Wendt, A. T. Santhanam, P. L. Hegde, and R. M. Penich. A new class of high performance pvd coatings for carbide cutting tools. Surface and Coatings Technology, 139(1):25 - 34, 2001.

[57] M. Amon, A. Bolz, and M. Schaldach. Improvement of stenting therapy with a silicon carbide coated tantalum stent. Journal of Materials Science: Materials in Medicine, 7:273-278f, 1996.

[58] A. Bolz and M. Schaldach. Physical mechanisms of solid-protein interactions at the interface between amorphous silicon carbide and fibrinogen. Biomedizinische Technik, 37:244-253, 1992.

[59] D. E. Siegfried, D. Cook, and D. Glocker. Reactive cylindrical magnetron deposition of titanium nitride and zirconium nitride films. Society of Vacuum Coaters, pages 97-101, 1996.

[60] F. R. Lipsett and D. Faraday. The deposition of thin films of gold on cylindrical specimens by sputtering. British Journal of Applied Physics, 6:442-443, 1955.

[61] J. A. Thornton and V. L. Hedgcoth. Tubular hollow cathode sputtering onto substrates of complex shape. Journal of Vacuum Science and Technology, 12(1):93-97, 1975.

[62] C. M. Ward-Close and P. G. Partridge. A fibre coating process for advanced metalmatrix composites. Journal of Materials Science, 25:4315-4323, 2004. 
[63] D. D. Hass, Y. Marciano, and H. N. G. Wadley. Physical vapor deposition on cylindrical substrates. Surface and Coatings Technology, 185(2-3):283 - 291, 2004.

[64] C. Ducros, V. Benevent, and F. Sanchette. Deposition, characterization and machining performance of multilayer pvd coatings on cemented carbide cutting tools. Surface and Coatings Technology, 163-164:681 - 688, 2003.

[65] P. J. S. Buenconsejo, K. Ito, H. Y. Kim, and S. Miyazaki. High-strength superelastic ti-ni microtubes fabricated by sputter deposition. Acta Materialia, 56(9):2063 2072, 2008.

[66] M. Komatsubara, T. Namazu, H. Nagasawa, T. Tsurui, and S. Inoue. Cylindrical film deposition system for three-dimensional titanium-nickel shape memory alloy microstructure. Vacuum, 83:703-707, 2009.

[67] S. Snow and S. C. Jacobsen. Microfabrication processes on cylindrical substrates - part i: Material deposition and removal. Microelectronic Engineering, 83:2534$2542,2006$.

[68] S. Snow and S. C. Jacobsen. Microfabrication processes on cylindrical substrates part ii: Lithography and connections. Microelectronic Engineering, 84:11-20, 2007.

[69] R. Lima de Miranda, C. Zamponi, and E. Quandt. Fabrication of tini thin film stents. Smart Mater. Struct., 2009 - In Print.

[70] J. C. Anderson, K. D. Leaver, P. Leevers, and R. D. Rawlings. Materials Science for Engineers. Nelson Thornes Ltd, 2003.

[71] P. J. Goodhew, J. Humphreys, and R. Beanland. Electron Microscopy and Analysis. Taylor \& Francis, 2001.

[72] J. Frenzel, Z. Zhang, K. Neuking, and G. Eggeler. High quality vacuum induction melting of small quantities of niti shape memory alloys in graphite crucibles. Journal of Alloys and Compounds, 385:214-223, 2004. 
[73] J. Zhang. PhD thesis, University of Tsukuba, 2000.

[74] H. Rumpf, T. Walther, C. Zamponi, and E. Quandt. High ultimate tensile stress in nano-grained superelastic niti thin films. Materials Science and Engineering A, 2005 .

[75] C Zamponi and R. Lima de Miranda. Thin Film Shape Memory Alloys, chapter 15. Cambridge University Press, 2009, In Print.

[76] V. V. Martynov, A. D. Johnson, V. Gupta, and V. Galhotra. Microstructure, transformation characteristics and mechanical properties of thin tini films deposited from a planar magnetron source onto a cylindrical surface. J.Phys. IV France, 11:421-426, 2001.

[77] S. Dabral, G.-R. Yang, H. Bakhru, T.-M. Lu, and J. F. McDonald. Chromium as an adhesion promoter/diffusion barrier for cu on parylene. VMlC Conference, 1991.

[78] A. Zendehnam, M. Mirzaei, and M. Khaneghaie. Study of reflection power and surface roughness of $\mathrm{cu}$ nanolayers thin film with respect to various deposition rates of sputtering. Journal of Physics: Conference Series, 61:1326-1330, 2007.

[79] S. Stemmer, G. Duscher, C. Scheu, A. H. Heuer, and M. Ruehle. The reaction between a tini shape memory thin film and silicon. Materials Research, 12:1734$1740,1997$.

[80] W. L. Benard, H. Kahn, A. H. Heuer, and M. A. Huff. Thin-film shape-memory alloy actuated micropumps. Journal of Microelectromechanical systems, 7:245-251, 1998.

[81] K. R. C. Gisser, J. D. Busch, A. D. Johnson, and A. B. Ellis. Oriented nickeltitanium shape memory alloy films prepared by annealing during deposition. Appl. Phys. Lett., 62:1632, 1992.

[82] H. Fujita, M. Komatsu, T. Sakata, and N. Fujita. Nucleatlon of crystals in amorphous materials. Mater. Trans. JIM, 37:1350, 1996. 
[83] J.C Palmaz, CT Boyle, Banas C.E., R.W. Wiseman, and D. Marton. Endoluminal implantable stens-grafts, us patent 7491226, 2002.

[84] M. J. Vestel and D. S. Grummon. Precipitates and lamellar microstructures in niti films. Materials Science and Engineering A, 2003.

[85] M. J. Vestel, D. S. Grummon, R. Gronsky, and A. P. Pisano. Effect of temperature on the devitrification kinetics of niti films. Acta Materialia, 2003.

[86] J. Z. Chen and S. K. Wu. Crystallization behavior of r.f.-sputtered tini thin films. Thin Solid Films, 339:194-199, 1999.

[87] K. J. Laider. World Of Physical Chemistry. Oxford University Press, 1993.

[88] T. W. Duerig, D. E. Tolomeo, and M. Wholey. An overview of superelastic stent design. Min Invas Therש6-x0026; Allied Technol, 9:235-246, 2000.

[89] H. Rumpf, V. Wipperfürth, C. Zamponi, and E. Quandt. Near net-shape fabrication of superelastic niti devices by sputtering and photoetching. Materials Transactions, 47:523-526, 2006.

[90] C. Zamponi, H. Rumpf, C. Schmutz, and E. Quandt. Structuring of sputtered superelastic niti thin films by photolithography and etching. Materials Science and Engineering A, 2006.

[91] C. Zamponi, M. Koch, H. Rumpf, and E. Quandt. Method for coating a stent, WO $2007 / 093423$. 